\title{
New Scientific Contribution on the 2-D Subdomain Technique in Cartesian Coordinates: Taking into Account of Iron Parts
}

\author{
Frédéric Dubas ${ }^{1, *}$ and Kamel Boughrara ${ }^{2}$ \\ 1 Département ENERGIE, FEMTO-ST, CNRS, Univ. Bourgogne Franche-Comté, F90000 Belfort, France \\ 2 Laboratoire de Recherche en Electrotechnique (LRE-ENP), 16200 Algiers, Algeria; \\ kamel.boughrara@g.enp.edu.dz \\ * Correspondence: frederic.dubas@univ-fcomte.fr; Tel.: +33-38-457-8203
}

Academic Editor: Fazal M. Mahomed

Received: 17 January 2017; Accepted: 1 February 2017; Published: 10 February 2017

\begin{abstract}
The most significant assumptions in the subdomain technique (i.e., based on the formal resolution of Maxwell's equations applied in subdomain) is defined by: The iron parts (i.e., the teeth and the back-iron are considered to be infinitely permeable, i.e., $\mu_{\text {iron }} \rightarrow+\infty$, so that the saturation effect is neglected. In this paper, the authors present a new scientific contribution on improving of this method in two-dimensional (2-D) and in Cartesian coordinates by focusing on the consideration of iron. The subdomains connection is carried out in the two directions (i.e., $x$ - and $y$-edges). For example, the improvement was performed by solving magnetostatic Maxwell's equations for an air- or iron-cored coil supplied by a direct current. To evaluate the efficacy of the proposed technique, the magnetic flux density distributions have been compared with those obtained by the 2-D finite-element analysis (FEA). The semi-analytical results are in quite satisfying agreement with those obtained by the 2-D FEA, considering both amplitude and waveform.
\end{abstract}

Keywords: air- or iron-cored coil; Cartesian coordinates; Fourier analysis; two-dimensional; saturation effect; subdomain technique

\section{Introduction}

\subsection{Context of this Paper}

Generally, the modeling of the electromagnetic field distribution is a key step in the design process for developing electromechanical systems. Although there are many papers in this scientific domain, the modeling approach is still a challenging and attractive research topic. Some comprehensive reviews on the models of electrical machines for magnetic field calculations along with their advantages and disadvantages can be found in [1-6] and the accompanying references. The modeling techniques can thus be classified in various categories:

- $\quad$ Graphical method of Lehmann (1909) [7];

- $\quad$ Numerical methods (i.e., the finite-element, finite-difference or boundary-element analysis) [8-12];

- $\quad$ Electrical/Thermal/Magnetic equivalent circuit (EEC/TEC/MEC) [13-16];

- Schwarz-Christoffel (SC) mapping method [17-19];

- Maxwell-Fourier methods [10,18-22]: (i) Multi-layers models; (ii) Eigenvalues model; and (iii) Subdomain technique.

The graphical method of Lehmann, which determines the magnetic field distribution in all parts of an electrical machine even when the machine is saturated, has been forgotten to the detriment of other methods, mainly numerical. In the past few decades, numerical modeling techniques 
have been applied to electromechanical systems analysis. These methods are precise and take into account the exact/simplified geometry, the nonlinear $B(H)$ curve, the rotor motion, etc. The most accurate models are the three-dimensional numerical methods. Nevertheless, these approaches are time-consuming and not suitable for the optimization problems. In [23,24], it is possible to optimize electromagnetic systems from numerical methods. Nowadays, in order to reduce the computation time, hybrid numerical methods can be developed [25-27]. The actual design works are mainly based on (semi-)analytical models (i.e., EEC/TEC/MEC, SC mapping and Maxwell-Fourier methods). This type of model consists of $N$ interrelated analytical equations which must be solved numerically (a.k.a. semi-numerical models). For example, in Maxwell-Fourier methods, the unknown coefficients of the series are computed by solving a (non)linear matrix system. Indeed, under certain assumptions, these models have the advantage to be explicit/accurate/fast. Moreover, they allow us to take into account rigorously the slotting effect in the electrical machines as well as various electromagnetic domains with(out) the current penetration effect in the conductive materials. Except in the numerical methods and nonlinear MEC, the saturation effect remains one of the scientific challenges in the modeling. Tiegna et al. [5] (p.168) wrote: "No examples of analytical models based on the formal solution of Maxwell's equations which take into account local magnetic saturation are available to date". Thus, in this paper, the main scientific focus will be on the consideration of iron in Maxwell-Fourier methods with the local/global saturation.

\subsection{State-of-the-Art: Saturation in Maxwell-Fourier Methods}

Very few works have included the iron in Maxwell-Fourier methods with the local/global saturation due to variation of the material properties (e.g., in case of stator and/or rotor slotting, buried magnets, etc.). The most significant assumptions is defined by: The iron parts (i.e., the teeth and the back-iron) are considered to the infinitely permeable, i.e., $\mu_{\text {iron }} \rightarrow+\infty$, so that the saturation effect is neglected. It results in an overestimation of the magnetic flux and, consequently, the electromagnetic performances (e.g., the back electromotive force (EMF), the electromagnetic torque, the efficiency). Thus, consideration of iron in the modeling is a mandatory task in order to have a reliable estimation of the electromechanical systems behavior.

Existing models in the electrical machines, based on Maxwell's electromagnetic field equations, taking into account the iron parts with(out) the nonlinear $B(H)$ curve are:

- Multi-layers models (only the global saturation):

- Carter's coefficient: The slotted machine is transformed into a slotless equivalent structure by applying the usual Carter's coefficient [28]. Generally, the armature slotting is taken into account through the SC mapping method. The analytical magnetic field distribution is determined in five or six homogeneous layers (i.e., exterior, slotless stator, winding/air-gap, magnets, and rotor) [29-31]. In [29], the magnetic permeabilities in stator/rotor iron cores have a constant value corresponding to linear zone of the $B(H)$ curve. An iterative technique to include the nonlinear properties of core material has been developed in [30] (for a no-load operation) and [31] (for a load operation whose the source term in the slot caused by the armature currents is represented by a winding current region over the stator slot-isthmus). In this type of modeling, the local distribution of flux densities in the teeth and slots is neglected. However, by calculating the flux entering the stator surface from the air-gap magnetic field and thus assuming uniform distribution of flux, the flux density in middle of the stator teeth can be obtained.

- Saturation coefficient: It represents the ratio between the total magnetomotive force (MMF) required for the entire magnetic circuit and the air-gap MMF [32]. The main magnetic saturation is included in the saturation factor, in an iterative way, by using the nonlinear $B(H)$ curve. The saturation effect is accounted for by modifying the air-gap length [32-34] or by changing the physical properties of magnets (in this case, the saturated load operation is 
calculated by considering an equivalent no-load operation with a fictitious magnet having a remanent flux density that creates the same MMF as the one created by both real magnet and stator MMF) [35]. The analytical magnetic field distribution is mainly determined in one or two regions (viz., air-gap or air-gap/magnets) of slotless machines by applying the Carter's coefficient [32]. The slotting effect can be neglected $[32,35]$ or taken into account through the SC mapping method $[33,34]$. The magnetic fluxes in the stator/rotor iron cores are obtained from the air-gap magnetic field [32,33,35] or/and with a simple MEC [34]. This technique has been applied to surface-mounted/-inset magnets machines [32-35], surface-inset magnet machines [33], and others electrical machines.

- Concept wave impedance: They are based on a direct solution of Maxwell's field equations in homogeneous multi-layers of magnetic material properties, viz., the magnetic permeability and the electrical conductivity. This approach, developed by Mishkin (1953) [36], was first applied to squirrel-cage induction machine in Cartesian coordinates with three-layers (i.e., stator slotting, air-gap, and rotor slotting). It was used and enhanced by many authors, viz.,

* simplification of the electromagnetic theory [37];

* extended with an infinite number of layers [38];

* converted into equivalent circuits and terminal impedance [39];

* included the curvature effect with the magnetizing current [40];

* incorporated spatial harmonics in the multi-layers theory by considering isotropic and anisotropic (e.g., laminated, composite, and toothed) regions [41,42];

* introduced the nonlinear $B(H)$ curve in homogenous layers by an iterative procedure $[43,44]$;

* taking account of the slot-opening effect [45], i.e., the multi-layers model is combined with the subdomain technique for slotted structures by assuming infinitely permeable tooth-tips;

* included the current penetration effect in conductive layers [43,46]. The analytical solution for the electromagnetic field in conductive layers is then defined by Bessel functions.

- Convolution theorem: The electrical machine is divided into an infinite number of (in)homogeneous layers. The permeability in the stator and/or rotor slotting is represented by a complex Fourier series along the direction of permeability variation The permeability variation in the direction of the periodicity is directly included into the solution of the magnetic field equation. The resulting formulation, based on a direct solution of Maxwell's field equations using the Cauchy's product theorem (i.e., the discrete convolution of two infinite series), is completely defined in terms of complex Fourier series [47]. Recently, Djelloul et al. (2016) [48] extended this modeling taking into account the nonlinear $B(H)$ curve in each soft-magnetic section by an iterative procedure. For the moment, this technique has been applied to a switched reluctance machine [48] and a synchronous reluctance machine [49].

- Eigenvalues model (only the global saturation): The electromagnetic field can be solved directly by applying the method of Truncation Region Eigenfunction Expansions (TREE) [50]. The iron cores have finite magnetic permeability and finite height/width. The studied domains can be (non)conductive. The boundary value problem is formulated in terms of the magnetic vector potential, which is expanded in a series of appropriate eigenfunctions. The unknown coefficients of the series are computed by solving a matrix system (by using a standard method such as the lower upper decomposition), which is formed by applying the usual interface conditions. The corresponding eigenvalues are the real roots of a function with the geometrical and the magnetic permeability of the core as parameters. Nevertheless, an iterative numerical method (e.g., the bisection [50] and Newton-Raphson [51] method) is always adopted to compute the discrete eigenvalues in both the odd and even parity solutions. For the moment, this technique has been 
widely applied to the non-destructive testing of conductive materials (e.g., for the I-cored [50] and E-cored [52,53] probes, for a long coil with a slot in a conductive plate [51], etc.).

- Hybrid models (the local/global saturation): The analytical solution can be combined with numerical methods [54-57] or (non)linear MEC [58-67]. Usually, the analytical solution is established in uniform regions of very low permeability (e.g., air-gap, and magnets) and other methods are sought in regions where magnetic saturation cannot be neglected (i.e., the stator and/or rotor iron cores).

\subsection{Objectives of this Paper}

To the best of the author's knowledge, in the literature, there is no (semi-)analytical model based on the subdomain technique that taking into account of iron parts with(out) the nonlinear $B(H)$ curve. Thus, the work in this paper takes part in the development and improvement of the subdomain technique on this scientific topic.

The disadvantage of multi-layers models, except those with the convolution theorem, is that they do not give a very accurate description of the local magnetic field distribution in the iron parts with a global saturation. In the harmonic modeling technique using the convolution theorem, convergence problems due to the truncated Fourier series around the soft-magnetic material discontinuities may exist [47-49]. Except in multi-layers models using the conception wave impedance and in the TREE method, the electrical conductivity is assumed to be zero. It is interesting to note that the TREE method is not similar to the novel method proposed in this paper. The difference is that TREE method imposes a term-by-term field continuity on one direction and a weak continuity on the other direction, while the 2-D subdomain technique imposes a weak continuity on both directions. Furthermore, the latter method does not need to find any special eigenvalues by using iterative numerical schemes. Contrary to the TREE method, the new approach proposed in this paper allows to decompose the analytical solution in Fourier series into two solutions according to the two directions and to respect the boundary conditions by applying the principle of superposition on the magnetic quantities. Moreover, it also allows evaluation of the local distribution of flux densities in the iron parts with a global saturation, does not have numerical convergence problems, and would easily introduce the current penetration effect in the conductive materials. Section 2 presents this new scientific contribution based on the subdomain technique. For example, it was performed by solving 2-D magnetostatic Maxwell's equations in Cartesian coordinates $(x, y)$ for an air- or iron-cored coil supplied by a direct current. The subdomains connection is carried out in the two directions (i.e., $x$ - and $y$-edges). The iron magnetic permeability is constant corresponding to linear zone of the initial magnetization curve. Nevertheless, as in [48], it should be mentioned that the material properties could be updated iteratively to take the nonlinear $B(H)$ curve of the material into account. However, this is beyond the scope of the paper. In Section 3, in order to evaluate the efficacy of the proposed technique, the magnetic flux density distributions have been compared with those obtained by the 2-D finite-element analysis (FEA) [8]. The comparisons are very satisfying in amplitudes and waveforms.

This major scientific contribution could be applied to rotating and/or linear electrical machines with(out) magnets supplied by a direct or alternate current (with any waveforms) whose the analysis would be based on a 2-D semi-analytical model in Cartesian coordinates (e.g., plane linear machines, axial-flux machines, etc.).

\section{A 2-D Subdomain Technique of Magnetic Field}

\subsection{Problem Description and Assumptions}

The application example, namely an air- or iron-cored coil, with the geometrical and physical parameters is illustrated in Figure 1. The system consists of a coil with $N_{t}$ turns of the copper wire which is supply by a direct current $I$. The direction of current in the conductor is defined by $\otimes$ for the forward conductor and $\odot$ for return conductor. The material in the middle of the coil can be air or iron. The system is surrounded by the vacuum via an infinite box. 


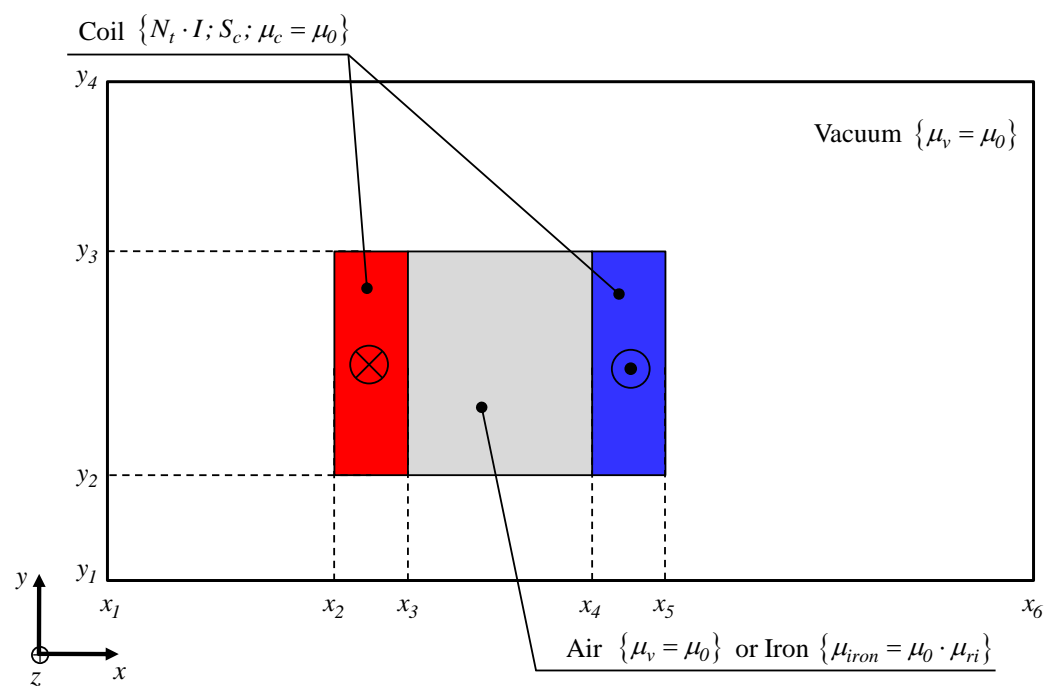

Figure 1. Air- or iron-cored coil (see Table 1 for the various parameters).

The 2-D magnetic field distribution in the air- or iron-cored coil has been studied in Cartesian coordinates $(x, y)$ by solving magnetostatic Maxwell's equations from subdomain technique. In this analysis, the magnetic field solution is based on the following simplifying assumptions:

- $\quad$ The end-effects are neglected (i.e., that the magnetic variables are independent of $z$ );

- The electrical conductivities of materials are assumed to be null (i.e., the eddy-currents induced in the copper/iron are neglected);

- The magnetic materials are considered as isotropic (i.e., the permeability can be assumed the same in the two directions);

- The effect of global saturation is taken into account with a constant magnetic permeability corresponding to linear zone of the $B(H)$ curve (i.e., the initial magnetization curve).

\subsection{Problem Discretization in Subdomains}

As shown in Figure 2, the problem domain is divided into 7 subdomains with $\mu=C^{s t}$. The vacuum around to the air- or iron-cored coil is defined by 4 regions, i.e.,

- Region $1\left\{\forall x \wedge y \in\left[y_{1}, y_{2}\right]\right\}$ with $\mu_{1}=\mu_{v}$;

- $\operatorname{Region} 2\left\{\forall x \wedge y \in\left[y_{3}, y_{4}\right]\right\}$ with $\mu_{2}=\mu_{v}$;

- Region $3\left\{x \in\left[x_{1}, x_{2}\right] \wedge y \in\left[y_{2}, y_{3}\right]\right\}$ with $\mu_{3}=\mu_{v}$;

- $\operatorname{Region} 4\left\{x \in\left[x_{5}, x_{6}\right] \wedge y \in\left[y_{2}, y_{3}\right]\right\}$ with $\mu_{4}=\mu_{v}$.

The air or iron in the middle of the coil is defined by the Region $5\left\{x \in\left[x_{2}, x_{3}\right] \wedge y \in\left[y_{2}, y_{3}\right]\right\}$ with $\mu_{5}=\mu_{v}$ for the air or $\mu_{5}=\mu_{\text {iron }}$ for the iron. The coil (i.e., forward and return conductors) is defined by 2 regions, i.e.,

- $\operatorname{Region} 6\left\{x \in\left[x_{2}, x_{3}\right] \wedge y \in\left[y_{2}, y_{3}\right]\right\}$ with $\mu_{6}=\mu_{c}$;

- $\operatorname{Region} 7\left\{x \in\left[x_{4}, x_{5}\right] \wedge y \in\left[y_{2}, y_{3}\right]\right\}$ with $\mu_{7}=\mu_{c}$.

\subsection{Governing Partial Differential Equations in Cartesian Coordinates}

According to (A4) (see Appendix A), the 2-D magnetic vector potential distribution in Cartesian coordinates $(x, y)$ is governed by the Laplace's equation in regions $j$ with $j=\{1, \ldots, 5\}$, i.e.,

$$
\Delta A_{z j}=\frac{\partial^{2} A_{z j}}{\partial x^{2}}+\frac{\partial^{2} A_{z j}}{\partial y^{2}}=0
$$




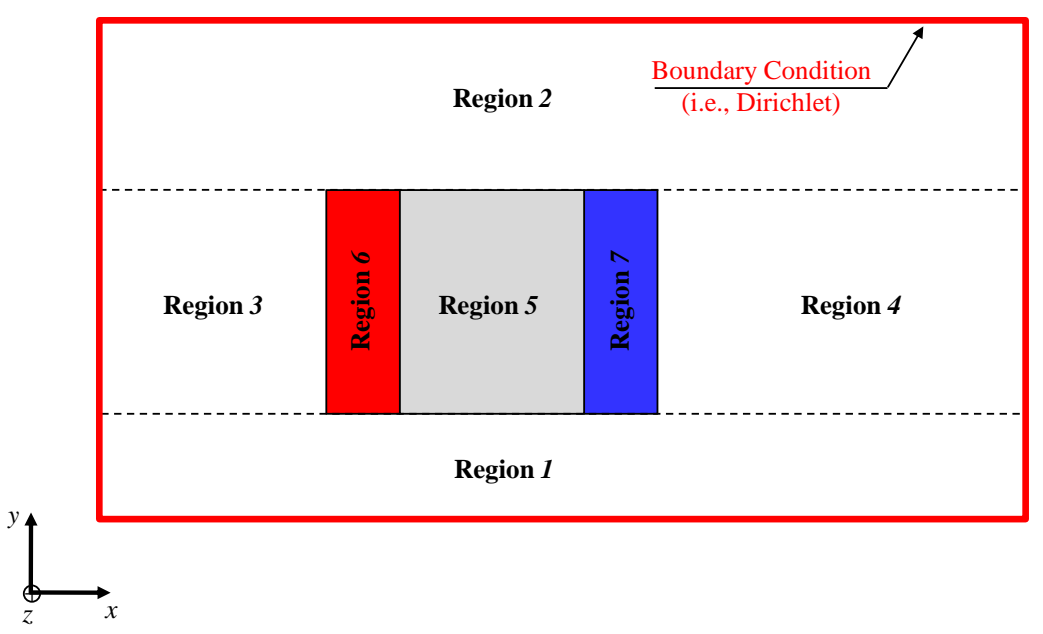

Figure 2. Subdomains in the air- or iron-cored coil.

and the Poisson's equation in regions $k$ with $k=\{6,7\}$, i.e.,

$$
\Delta A_{z k}=\frac{\partial^{2} A_{z k}}{\partial x^{2}}+\frac{\partial^{2} A_{z k}}{\partial y^{2}}=-\mu_{k} \cdot J_{z k}
$$

where $J_{z k}$ represents the current density (due to supply currents) which is defined by

$$
J_{z k}=C_{k} \cdot \frac{N_{t} \cdot I}{S_{c}}
$$

in which $S_{c}$ is the conductor surface, and $C_{k}$ is the coefficient for the direction of current in the conductor (e.g., with $C_{6}=1$ for the forward conductor and $C_{7}=-1$ for return conductor).

According to the method of separation of variables, it is interesting to note that $A_{z} \bullet$ can be decomposed into two potentials according to the two directions (see Appendix A), i.e., $A_{z \bullet}^{x}$ for the $x$-edges (A5b) and $A_{z \bullet}^{y}$ for the $y$-edges (A5c). The periodicity of $A_{z \bullet}^{x}$ and $A_{z \bullet}^{y}$ are respectively defined by $\beta \bullet_{\bullet}$ and $\lambda \bullet \bullet_{n} \bullet$ with $h \bullet$ and $n \bullet$ the spatial harmonic orders.

\subsection{Boundary Conditions}

\subsubsection{Reminder on the Boundary Conditions at the Interface of Two Surfaces}

In electromagnetic, as shown in Figure 3 , the magnetic field $\vec{H}$ obeys Ampère's continuity condition,

$$
\vec{n} \times\left(\vec{H}_{\| a}-\vec{H}_{\| b}\right)=\vec{K},
$$

where $\vec{n}$ is the unit vector normal to the boundary between two surfaces, $\vec{H}_{\|}$the parallel component of $\vec{H}$ on one side of the interface, and $\vec{K}$ the current density at the surface of the interface.

At this same surface, the magnetic flux continuity condition also applies

$$
\vec{n} \cdot\left(\vec{B}_{\perp a}-\vec{B}_{\perp b}\right)=0 \text { or } \vec{A}_{a}-\vec{A}_{b}=0,
$$

where $\vec{B}_{\perp}$ is the perpendicular component of $\vec{B}$ on one side of the interface. The Dirichlet condition on one surface is defined by

$$
\vec{A}_{a}=0 \text { or } \vec{A}_{b}=0 .
$$




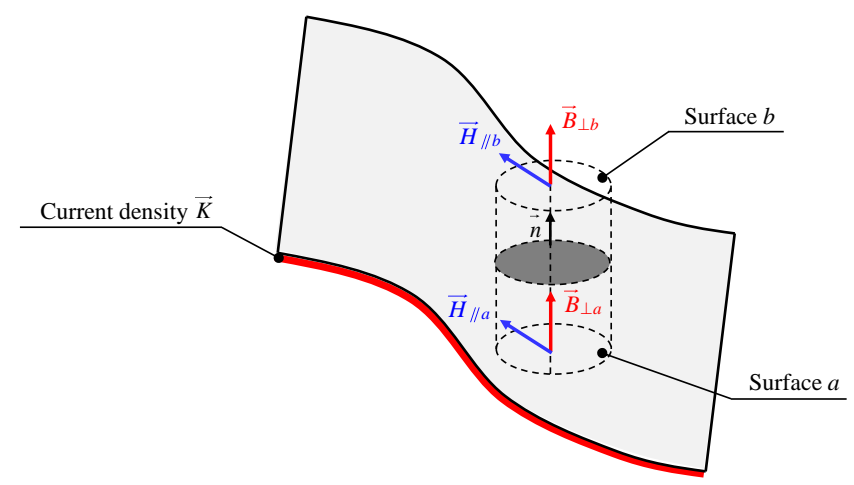

Figure 3. Boundary conditions at the interface of two surfaces.

\subsubsection{Application to the Air- or Iron-Cored Coil}

On the outer boundaries for $\left(x_{1} \wedge x_{6}, \forall y\right)$ and $\left(\forall x, y_{1} \wedge y_{4}\right)$ (see Figure 2$)$, the component of the magnetic vector potential satisfies the Dirichlet boundary condition, i.e., (3c). By applying (3) and using (A2) (see Appendix A), the respective boundaries at the interface between the various regions are illustrated in Figure 4.

\subsection{General Solutions}

\subsubsection{Region 1}

The general solution of $A_{z 1}, B_{x 1}$ and $B_{y 1}$ are determined by the particular case of the case-study no 1 (i.e., $A_{z}$ imposed on all edges of a region) in Appendix B. The boundary conditions on the $y$-edges of the region (see Figure 4a) are met by posing $c_{h}^{x}=0$ in (B4). Therefore, the magnetic vector potential $A_{z 1}$, which is a solution of (1) satisfying the boundary conditions of Figure 4a, is defined by

$$
A_{z 1}=\sum_{h 1=1}^{\infty} \frac{d 1_{h 1}^{x}}{\beta 1_{h 1}} \cdot \frac{\operatorname{sh}\left[\beta 1_{h 1} \cdot\left(y-y_{1}\right)\right]}{\operatorname{ch}\left(\beta 1_{h 1} \cdot \tau_{y 1}\right)} \cdot \sin \left[\beta 1_{h 1} \cdot\left(x-x_{1}\right)\right]
$$

the components of $\vec{B}_{1}=\left\{B_{x 1} ; B_{y 1} ; 0\right\}$ by

$$
\begin{gathered}
B_{x 1}=\sum_{h 1=1}^{\infty} d 1_{h 1}^{x} \cdot \frac{c h\left[\beta 1_{h 1} \cdot\left(y-y_{1}\right)\right]}{c h\left(\beta 1_{h 1} \cdot \tau_{y 1}\right)} \cdot \sin \left[\beta 1_{h 1} \cdot\left(x-x_{1}\right)\right], \\
B_{y 1}=-\sum_{h 1=1}^{\infty} d 1_{h 1}^{x} \cdot \frac{\operatorname{sh}\left[\beta 1_{h 1} \cdot\left(y-y_{1}\right)\right]}{c h\left(\beta 1_{h 1} \cdot \tau_{y 1}\right)} \cdot \cos \left[\beta 1_{h 1} \cdot\left(x-x_{1}\right)\right],
\end{gathered}
$$

where $h 1$ is the spatial harmonic orders in Region $1, d 1_{h 1}^{x}$ the integration constant, $\beta 1_{h 1}=h 1 \cdot \pi / \tau_{x 1}$ with $\tau_{x 1}=x_{6}-x_{1}$, and $\tau_{y 1}=y_{2}-y_{1}$.

The coefficient $d 1_{h 1}^{x}$ is determined using a Fourier series expansion of $F_{1}(x)$ (see Figure 4a) over the interval $x=\left[x_{1}, x_{6}\right]=\left[x_{1}, x_{1}+\tau_{x 1}\right]$ :

$$
d 1_{h 1}^{x}=\frac{2}{\tau_{x 1}} \cdot \int_{x_{1}}^{x_{1}+\tau_{x 1}} F_{1}(x) \cdot \sin \left[\beta 1_{h 1} \cdot\left(x-x_{1}\right)\right] \cdot d x .
$$

The expression of $d 1_{h 1}^{x}$ is developed in Appendix C. 


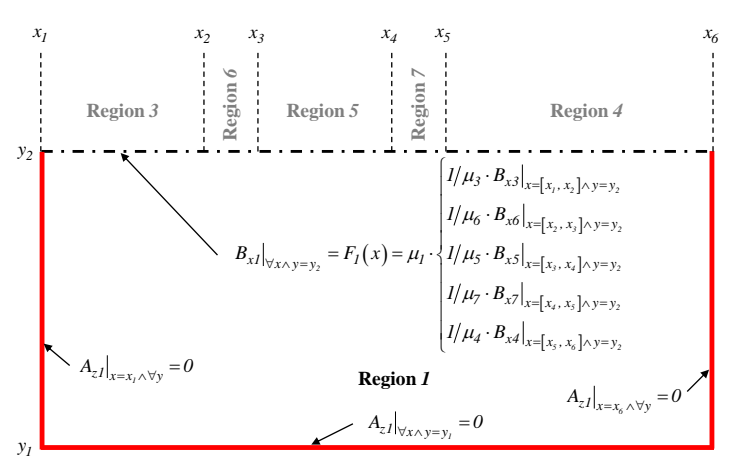

(a)

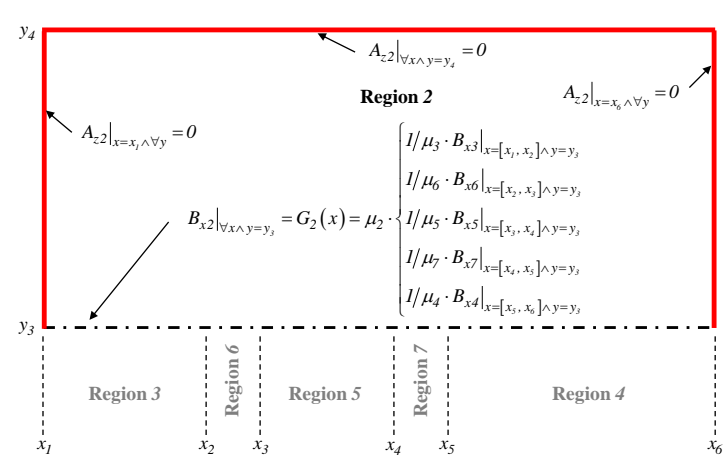

(b)

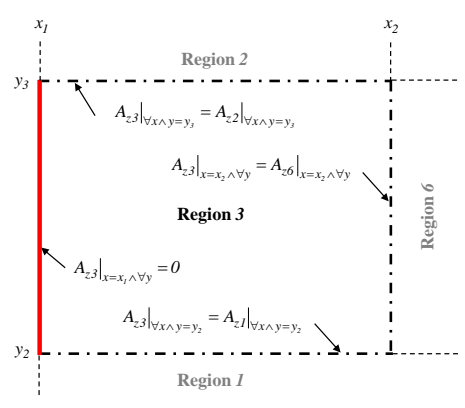

(c)

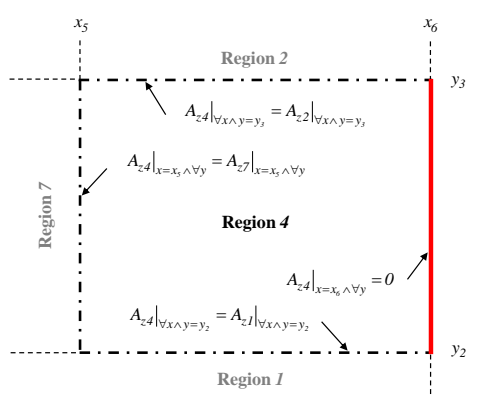

(d)

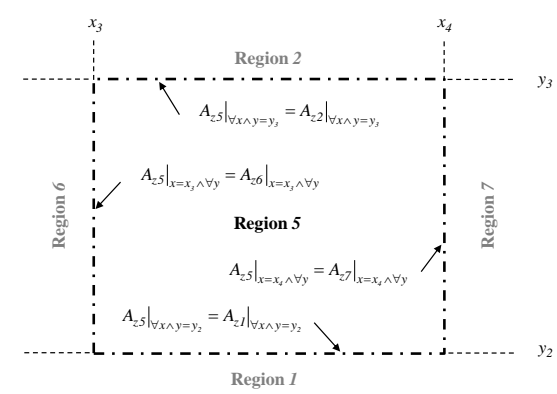

(e)

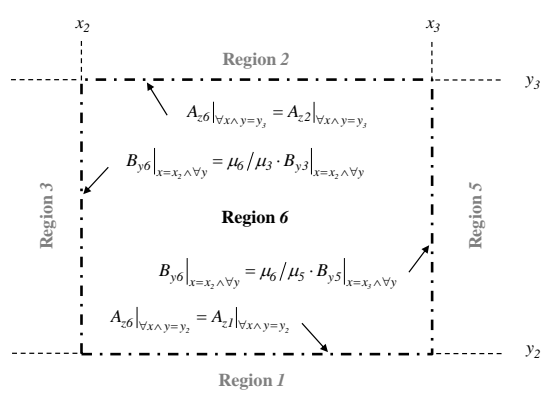

(f)

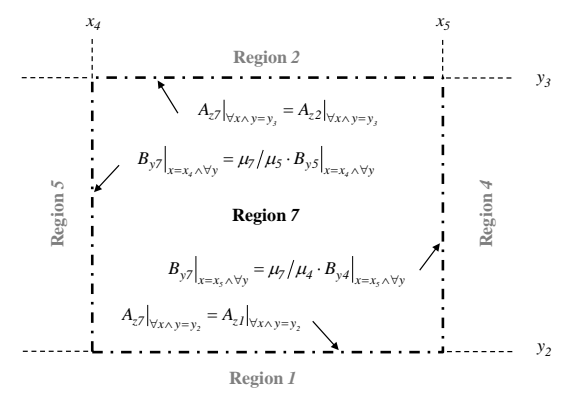

(g)

Figure 4. Boundary conditions in subdomains: (a) Region 1; (b) Region 2; (c) Region 3; (d) Region 4; (e) Region 5; (f) Region 6; and (g) Region 7.

\subsubsection{Region 2}

The same method than Region 1 is used to find the solution in Region 2. By posing $d_{h}^{x}=0$ in (B4) (see Appendix B), the magnetic vector potential $A_{z 2}$, which is a solution of (1) satisfying the boundary conditions of Figure $4 b$, is defined by

$$
A_{z 2}=-\sum_{h 2=1}^{\infty} \frac{c 2_{h 2}^{x}}{\beta 2_{h 2}} \cdot \frac{\operatorname{sh}\left[\beta 2_{h 2} \cdot\left(y_{4}-y\right)\right]}{c h\left(\beta 2_{h 2} \cdot \tau_{y 2}\right)} \cdot \sin \left[\beta 2_{h 2} \cdot\left(x-x_{1}\right)\right]
$$

the components of $\vec{B}_{2}=\left\{B_{x 2} ; B_{y 2} ; 0\right\}$ by

$$
B_{x 2}=\sum_{h 2=1}^{\infty} c 2_{h 2}^{x} \cdot \frac{\operatorname{ch}\left[\beta 2_{h 2} \cdot\left(y_{4}-y\right)\right]}{c h\left(\beta 2_{h 2} \cdot \tau_{y 2}\right)} \cdot \sin \left[\beta 2_{h 2} \cdot\left(x-x_{1}\right)\right],
$$




$$
B_{y 2}=\sum_{h 2=1}^{\infty} c 2_{h 2}^{x} \cdot \frac{s h\left[\beta 2_{h 2} \cdot\left(y_{4}-y\right)\right]}{c h\left(\beta 2_{h 2} \cdot \tau_{y 2}\right)} \cdot \cos \left[\beta 2_{h 2} \cdot\left(x-x_{1}\right)\right],
$$

where $h 2$ is the spatial harmonic orders in Region $2, c 2_{h 2}^{x}$ the integration constant, $\beta 2_{h 2}=h 2 \cdot \pi / \tau_{x 2}$ with $\tau_{x 2}=x_{6}-x_{1}$, and $\tau_{y 2}=y_{4}-y_{3}$.

The coefficient $c 2_{h 2}^{x}$ is determined using a Fourier series expansion of $G_{2}(x)$ (see Figure $4 \mathrm{~b}$ ) over the interval $x=\left[x_{1}, x_{6}\right]=\left[x_{1}, x_{1}+\tau_{x 2}\right]$ :

$$
c 2_{h 2}^{x}=\frac{2}{\tau_{x 2}} \cdot \int_{x_{1}}^{x_{1}+\tau_{x 2}} G_{2}(x) \cdot \sin \left[\beta 2_{h 2} \cdot\left(x-x_{1}\right)\right] \cdot d x .
$$

The expression of $c 2_{h 2}^{x}$ is developed in Appendix C.

\subsubsection{Region 3}

The general solution of $A_{z 3}, B_{x 3}$ and $B_{y 3}$ are determined by the case-study no 1 (i.e., $A_{z}$ imposed on all edges of a region) in Appendix $B$. The boundary conditions on the $x$-edges of the region (see Figure 4c) are met by posing $e_{n}^{y}=0$ in (B1)-(B3). Therefore, the magnetic vector potential $A_{z 3}$, which is a solution of (1) satisfying the boundary conditions of Figure $4 \mathrm{c}$, is defined by

$$
\begin{gathered}
A_{z 3}=A_{z 3}^{x}+A_{z 3^{\prime}}^{y} \\
A_{z 3}^{x}=\sum_{h 3=1}^{\infty}\left\{\frac{c 3_{h 3}^{x}}{\beta 3_{h 3}} \cdot \frac{s h\left[\beta 3_{h 3} \cdot\left(y_{3}-y\right)\right]}{\operatorname{sh}\left(\beta 3_{h 3} \cdot \tau_{y 3}\right)}+\frac{d 3_{h 3}^{x}}{\beta 3_{h 3}} \cdot \frac{\operatorname{sh}\left[\beta 3_{h 3} \cdot\left(y-y_{2}\right)\right]}{\operatorname{sh}\left(\beta 3_{h 3} \cdot \tau_{y 3}\right)}\right\} \cdot \sin \left[\beta 3_{h 3} \cdot\left(x-x_{1}\right)\right], \\
A_{z 3}^{y}=\sum_{n 3=1}^{\infty} \frac{f 3_{n 3}^{y}}{\lambda 3_{n 3}} \cdot \frac{\operatorname{sh}\left[\lambda 3_{n 3} \cdot\left(x-x_{1}\right)\right]}{\operatorname{sh}\left(\lambda 3_{n 3} \cdot \tau_{x 3}\right)} \cdot \sin \left[\lambda 3_{n 3} \cdot\left(y-y_{2}\right)\right],
\end{gathered}
$$

the $x$-component of $\vec{B}_{3}$ by

$$
\begin{gathered}
B_{x 3}=B_{x 3}^{x}+B_{x 3^{\prime}}^{y} \\
B_{x 3}^{x}=\sum_{h 3=1}^{\infty}\left\{-c 3_{h 3}^{x} \cdot \frac{\operatorname{ch}\left[\beta 3_{h 3} \cdot\left(y_{3}-y\right)\right]}{\operatorname{sh}\left(\beta 3_{h 3} \cdot \tau_{y 3}\right)}+d 3_{h 3}^{x} \cdot \frac{\operatorname{ch}\left[\beta 3_{h 3} \cdot\left(y-y_{2}\right)\right]}{\operatorname{sh}\left(\beta 3_{h 3} \cdot \tau_{y 3}\right)}\right\} \cdot \sin \left[\beta 3_{h 3} \cdot\left(x-x_{1}\right)\right], \\
B_{x 3}^{y}=\sum_{n 3=1}^{\infty} f 3_{n 3}^{y} \cdot \frac{\operatorname{sh}\left[\lambda 3_{n 3} \cdot\left(x-x_{1}\right)\right]}{\operatorname{sh}\left(\lambda 3_{n 3} \cdot \tau_{x 3}\right)} \cdot \cos \left[\lambda 3_{n 3} \cdot\left(y-y_{2}\right)\right],
\end{gathered}
$$

the $y$-component of $\vec{B}_{3}$ by

$$
\begin{gathered}
B_{y 3}=B_{y 3}^{x}+B_{y 3}^{y} \\
B_{y 3}^{x}=-\sum_{h 3=1}^{\infty}\left\{c 3_{h 3}^{x} \cdot \frac{\operatorname{sh}\left[\beta 3_{h 3} \cdot\left(y_{3}-y\right)\right]}{\operatorname{sh}\left(\beta 3_{h 3} \cdot \tau_{y 3}\right)}+d 3_{h 3}^{x} \cdot \frac{\operatorname{sh}\left[\beta 3_{h 3} \cdot\left(y-y_{2}\right)\right]}{\operatorname{sh}\left(\beta 3_{h 3} \cdot \tau_{y 3}\right)}\right\} \cdot \cos \left[\beta 3_{h 3} \cdot\left(x-x_{1}\right)\right], \\
B_{y 3}^{y}=-\sum_{n 3=1}^{\infty} f 3_{n 3}^{y} \cdot \frac{\operatorname{ch}\left[\lambda 3_{n 3} \cdot\left(x-x_{1}\right)\right]}{\operatorname{sh}\left(\lambda 3_{n 3} \cdot \tau_{x 3}\right)} \cdot \sin \left[\lambda 3_{n 3} \cdot\left(y-y_{2}\right)\right],
\end{gathered}
$$

where $h 3$ and $n 3$ are the spatial harmonic orders in Region $3 ; c 3_{h 3}^{x}, d 3_{h 3}^{x}$ and $f 3_{n 3}^{x}$ the integration constants; $\beta 3_{h 3}=h 3 \cdot \pi / \tau_{x 3}$ with $\tau_{x 3}=x_{2}-x_{1}$; and $\lambda 3_{n 3}=n 3 \cdot \pi / \tau_{y 3}$ with $\tau_{y 3}=y_{3}-y_{2}$.

The coefficients $c 3_{h 3}^{x}$ and $d 3_{h 3}^{x}$ are respectively determined using Fourier series expansion of $\left.A_{z 1}\right|_{\forall x \wedge y=y_{2}}$ and $\left.A_{z 2}\right|_{\forall x \wedge y=y_{3}}$ (see Figure 4c) over the interval $x=\left[x_{1}, x_{2}\right]=\left[x_{1}, x_{1}+\tau_{x 3}\right]$ :

$$
c 3_{h 3}^{x}=\left.\frac{2}{\tau_{x 3}} \cdot \int_{x_{1}}^{x_{1}+\tau_{x 3}} \beta 3_{h 3} \cdot A_{z 1}\right|_{y=y_{2}} \cdot \sin \left[\beta 3_{h 3} \cdot\left(x-x_{1}\right)\right] \cdot d x,
$$




$$
d 3_{h 3}^{x}=\left.\frac{2}{\tau_{x 3}} \cdot \int_{x_{1}}^{x_{1}+\tau_{x 3}} \beta 3_{h 3} \cdot A_{z 2}\right|_{y=y_{3}} \cdot \sin \left[\beta 3_{h 3} \cdot\left(x-x_{1}\right)\right] \cdot d x .
$$

The coefficient $f 3_{n 3}^{y}$ is determined using a Fourier series expansion of $\left.A_{z 6}\right|_{x=x_{2} \wedge \forall y}$ (see Figure 4c) over the interval $y=\left[y_{2}, y_{3}\right]=\left[y_{2}, y_{2}+\tau_{y_{3}}\right]$ :

$$
f 3_{n 3}^{y}=\left.\frac{2}{\tau_{y 3}} \cdot \int_{y_{2}}^{y_{2}+\tau_{y 3}} \lambda 3_{n 3} \cdot A_{z 6}\right|_{x=x_{2}} \cdot \sin \left[\lambda 3_{n 3} \cdot\left(y-y_{2}\right)\right] \cdot d y .
$$

The expression of $c 3_{h 3}^{x}, d 3_{h 3}^{x}$ and $f 3_{n 3}^{y}$ are developed in Appendix C.

\subsubsection{Region 4}

The same method than Region 3 is used to find the solution in Region 4 . By posing $f_{n}^{y}=0$ in (B1)-(B3) (see Appendix B), the magnetic vector potential $A_{z 4}$, which is a solution of (1) satisfying the boundary conditions of Figure $4 \mathrm{~d}$, is defined by

$$
\begin{gathered}
A_{z 4}=A_{z 4}^{x}+A_{z 4^{\prime}}^{y} \\
A_{z 4}^{x}=\sum_{h 4=1}^{\infty}\left\{\frac{c 4_{h 4}^{x}}{\beta 4_{h 4}} \cdot \frac{s h\left[\beta 4_{h 4} \cdot\left(y_{3}-y\right)\right]}{\operatorname{sh}\left(\beta 4_{h 4} \cdot \tau_{y 4}\right)}+\frac{d 4_{h 4}^{x}}{\beta 4_{h 4}} \cdot \frac{\operatorname{sh}\left[\beta 4_{h 4} \cdot\left(y-y_{2}\right)\right]}{\operatorname{sh}\left(\beta 4_{h 4} \cdot \tau_{y 4}\right)}\right\} \cdot \sin \left[\beta 4_{h 4} \cdot\left(x-x_{5}\right)\right], \\
A_{z 4}^{y}=\sum_{n 4=1}^{\infty} \frac{e 4_{n 4}^{y}}{\lambda 4_{n 4}} \cdot \frac{\operatorname{sh}\left[\lambda 4_{n 4} \cdot\left(x_{6}-x\right)\right]}{\operatorname{sh}\left(\lambda 4_{n 4} \cdot \tau_{x 4}\right)} \cdot \sin \left[\lambda 4_{n 4} \cdot\left(y-y_{2}\right)\right],
\end{gathered}
$$

the $x$-component of $\vec{B}_{4}$ by

$$
\begin{gathered}
B_{x 4}=B_{x 4}^{x}+B_{x 4^{\prime}}^{y} \\
B_{x 4}^{x}=\sum_{h 4=1}^{\infty}\left\{-c 4_{h 4}^{x} \cdot \frac{\operatorname{ch}\left[\beta 4_{h 4} \cdot\left(y_{3}-y\right)\right]}{\operatorname{sh}\left(\beta 4_{h 4} \cdot \tau_{y 4}\right)}+d 4_{h 4}^{x} \cdot \frac{\operatorname{ch}\left[\beta 4_{h 4} \cdot\left(y-y_{2}\right)\right]}{\operatorname{sh}\left(\beta 4_{h 4} \cdot \tau_{y 4}\right)}\right\} \cdot \sin \left[\beta 4_{h 4} \cdot\left(x-x_{5}\right)\right], \\
B_{x 4}^{y}=\sum_{n 4=1}^{\infty} e 4_{n 4}^{y} \cdot \frac{\operatorname{sh}\left[\lambda 4_{n 4} \cdot\left(x_{6}-x\right)\right]}{\operatorname{sh}\left(\lambda 4_{n 4} \cdot \tau_{x 4}\right)} \cdot \cos \left[\lambda 4_{n 4} \cdot\left(y-y_{2}\right)\right],
\end{gathered}
$$

the $y$-component of $\vec{B}_{4}$ by

$$
\begin{gathered}
B_{y 4}=B_{y 4}^{x}+B_{y 4^{\prime}}^{y} \\
B_{y 4}^{x}=-\sum_{h 4=1}^{\infty}\left\{c 4_{h 4}^{x} \cdot \frac{s h\left[\beta 4_{h 4} \cdot\left(y_{3}-y\right)\right]}{\operatorname{sh}\left(\beta 4_{h 4} \cdot \tau_{y 4}\right)}+d 4_{h 4}^{x} \cdot \frac{\operatorname{sh}\left[\beta 4_{h 4} \cdot\left(y-y_{2}\right)\right]}{\operatorname{sh}\left(\beta 4_{h 4} \cdot \tau_{y 4}\right)}\right\} \cdot \cos \left[\beta 4_{h 4} \cdot\left(x-x_{5}\right)\right], \\
B_{y 4}^{y}=\sum_{n 4=1}^{\infty} e 4_{n 4}^{y} \cdot \frac{\operatorname{ch}\left[\lambda 4_{n 4} \cdot\left(x_{6}-x\right)\right]}{\operatorname{sh}\left(\lambda 4_{n 4} \cdot \tau_{x 4}\right)} \cdot \sin \left[\lambda 4_{n 4} \cdot\left(y-y_{2}\right)\right]
\end{gathered}
$$

where $h 4$ and $n 4$ are the spatial harmonic orders in Region $4 ; c 4_{h 4^{\prime}}^{x} d 4_{h 4}^{x}$ and $e 4_{n 4}^{y}$ the integration constants; $\beta 4_{h 4}=h 4 \cdot \pi / \tau_{x 4}$ with $\tau_{x 4}=x_{6}-x_{5}$; and $\lambda 4_{n 4}=n 4 \cdot \pi / \tau_{y 4}$ with $\tau_{y 4}=y_{3}-y_{2}$.

The coefficients $c 4_{h 4}^{x}$ and $d 4_{h 4}^{x}$ are respectively determined using Fourier series expansion of $\left.A_{z 1}\right|_{\forall x \wedge y=y_{2}}$ and $\left.A_{z 2}\right|_{\forall x \wedge y=y_{3}}$ (see Figure 4d) over the interval $x=\left[x_{5}, x_{6}\right]=\left[x_{5}, x_{5}+\tau_{x 4}\right]$ :

$$
c 4_{h 4}^{x}=\left.\frac{2}{\tau_{x 4}} \cdot \int_{x_{5}}^{x_{5}+\tau_{x 4}} \beta 4_{h 4} \cdot A_{z 1}\right|_{y=y_{2}} \cdot \sin \left[\beta 4_{h 4} \cdot\left(x-x_{5}\right)\right] \cdot d x,
$$




$$
d 4_{h 4}^{x}=\left.\frac{2}{\tau_{x 4}} \cdot \int_{x_{5}}^{x_{5}+\tau_{x 4}} \beta 4_{h 4} \cdot A_{z 2}\right|_{y=y_{3}} \cdot \sin \left[\beta 4_{h 4} \cdot\left(x-x_{5}\right)\right] \cdot d x .
$$

The coefficient $e 4_{n 4}^{y}$ is determined using a Fourier series expansion of $\left.A_{z 7}\right|_{x=x_{5} \wedge \forall y}$ (see Figure 4d) over the interval $y=\left[y_{2}, y_{3}\right]=\left[y_{2}, y_{2}+\tau_{y 4}\right]$ :

$$
e 4_{n 4}^{y}=\left.\frac{2}{\tau_{y 4}} \cdot \int_{y_{2}}^{y_{2}+\tau_{y 4}} \lambda 4_{n 4} \cdot A_{z 7}\right|_{x=x_{5}} \cdot \sin \left[\lambda 4_{n 4} \cdot\left(y-y_{2}\right)\right] \cdot d y .
$$

The expression of $c 4_{h 4^{\prime}}^{x} d 4_{h 4}^{x}$ and $e 4_{n 4}^{y}$ are developed in Appendix C.

\subsubsection{Region 5}

According to case-study no 1 (i.e., $A_{z}$ imposed on all edges of a region) in Appendix $\mathrm{B}$, the magnetic vector potential $A_{z 5}$, which is a solution of (1) satisfying the boundary conditions of Figure 4e, is defined by

$$
\begin{gathered}
A_{z 5}=A_{z 5}^{x}+A_{z 5}^{y} \\
A_{z 5}^{x}=\sum_{h 5=1}^{\infty}\left\{\frac{c 5_{h 5}^{x}}{\beta 5_{h 5}} \cdot \frac{\operatorname{sh}\left[\beta 5_{h 5} \cdot\left(y_{3}-y\right)\right]}{\operatorname{sh}\left(\beta 5_{h 5} \cdot \tau_{y 5}\right)}+\frac{d 5_{h 5}^{x}}{\beta 5_{h 5}} \cdot \frac{\operatorname{sh}\left[\beta 5_{h 5} \cdot\left(y-y_{2}\right)\right]}{\operatorname{sh}\left(\beta 5_{h 5} \cdot \tau_{y 5}\right)}\right\} \cdot \sin \left[\beta 5_{h 5} \cdot\left(x-x_{3}\right)\right], \\
A_{z 5}^{y}=\sum_{n 5=1}^{\infty}\left\{\frac{e 5_{n 5}^{y}}{\lambda 5_{n 5}} \cdot \frac{\operatorname{sh}\left[\lambda 5_{n 5} \cdot\left(x_{4}-x\right)\right]}{\operatorname{sh}\left(\lambda 5_{n 5} \cdot \tau_{x 5}\right)}+\frac{f 5_{n 5}^{y}}{\lambda 5_{n 5}} \cdot \frac{\operatorname{sh}\left[\lambda 5_{n 5} \cdot\left(x-x_{3}\right)\right]}{\operatorname{sh}\left(\lambda 5_{n 5} \cdot \tau_{x 5}\right)}\right\} \cdot \sin \left[\lambda 5_{n 5} \cdot\left(y-y_{2}\right)\right],
\end{gathered}
$$

the $x$-component of $\vec{B}_{5}$ by

$$
\begin{gathered}
B_{x 5}=B_{x 5}^{x}+B_{x 5^{\prime}}^{y} \\
B_{x 5}^{x}=\sum_{h 5=1}^{\infty}\left\{-c 5_{h 5}^{x} \cdot \frac{\operatorname{ch}\left[\beta 5_{h 5} \cdot\left(y_{3}-y\right)\right]}{\operatorname{sh}\left(\beta 5_{h 5} \cdot \tau_{y 5}\right)}+d 5_{h 5}^{x} \cdot \frac{\operatorname{ch}\left[\beta 5_{h 5} \cdot\left(y-y_{2}\right)\right]}{\operatorname{sh}\left(\beta 5_{h 5} \cdot \tau_{y 5}\right)}\right\} \cdot \sin \left[\beta 5_{h 5} \cdot\left(x-x_{3}\right)\right], \\
B_{x 5}^{y}=\sum_{n 5=1}^{\infty}\left\{e 5_{n 5}^{y} \cdot \frac{\operatorname{sh}\left[\lambda 5_{n 5} \cdot\left(x_{4}-x\right)\right]}{\operatorname{sh}\left(\lambda 5_{n 5} \cdot \tau_{x 5}\right)}+f 5_{n 5}^{y} \cdot \frac{\operatorname{sh}\left[\lambda 5_{n 5} \cdot\left(x-x_{3}\right)\right]}{\operatorname{sh}\left(\lambda 5_{n 5} \cdot \tau_{x 5}\right)}\right\} \cdot \cos \left[\lambda 5_{n 5} \cdot\left(y-y_{2}\right)\right],
\end{gathered}
$$

the $y$-component of $\vec{B}_{5}$ by

$$
\begin{gathered}
B_{y 5}=B_{y 5}^{x}+B_{y 5}^{y} \\
B_{y 5}^{x}=-\sum_{h 5=1}^{\infty}\left\{c 5_{h 5}^{x} \cdot \frac{s h\left[\beta 5_{h 5} \cdot\left(y_{3}-y\right)\right]}{\operatorname{sh}\left(\beta 5_{h 5} \cdot \tau_{y 5}\right)}+d 5_{h 5}^{x} \cdot \frac{\operatorname{sh}\left[\beta 5_{h 5} \cdot\left(y-y_{2}\right)\right]}{\operatorname{sh}\left(\beta 5_{h 5} \cdot \tau_{y 5}\right)}\right\} \cdot \cos \left[\beta 5_{h 5} \cdot\left(x-x_{3}\right)\right], \\
B_{y 5}^{y}=-\sum_{n 5=1}^{\infty}\left\{-e 5_{n 5}^{y} \cdot \frac{c h\left[\lambda 5_{n 5} \cdot\left(x_{4}-x\right)\right]}{\operatorname{sh}\left(\lambda 5_{n 5} \cdot \tau_{x 5}\right)}+f 5_{n 5}^{y} \cdot \frac{\operatorname{ch}\left[\lambda 5_{n 5} \cdot\left(x-x_{3}\right)\right]}{\operatorname{sh}\left(\lambda 5_{n 5} \cdot \tau_{x 5}\right)}\right\} \cdot \sin \left[\lambda 5_{n 5} \cdot\left(y-y_{2}\right)\right],
\end{gathered}
$$

where $h 5$ and $n 5$ are the spatial harmonic orders in Region $5 ; c 5_{h 5}^{x}, d 5_{h 5}^{x}, e 5_{n 5}^{y}$ and $f 5_{n 5}^{y}$ the integration constants; $\beta 5_{h 5}=h 5 \cdot \pi / \tau_{x 5}$ with $\tau_{x 5}=x_{4}-x_{3}$; and $\lambda 5_{n 5}=n 5 \cdot \pi / \tau_{y 5}$ with $\tau_{y 5}=y_{3}-y_{2}$.

The coefficients $c 5_{h 5}^{x}$ and $d 5_{h 5}^{x}$ are respectively determined using Fourier series expansion of $\left.A_{z 1}\right|_{\forall x \wedge y=y_{2}}$ and $\left.A_{z 2}\right|_{\forall x \wedge y=y_{3}}$ (see Figure 4e) over the interval $x=\left[x_{3}, x_{5}\right]=\left[x_{3}, x_{3}+\tau_{x 5}\right]$ :

$$
c 5_{h 5}^{x}=\left.\frac{2}{\tau_{x 5}} \cdot \int_{x_{3}}^{x_{3}+\tau_{x 5}} \beta 5_{h 5} \cdot A_{z 1}\right|_{y=y_{2}} \cdot \sin \left[\beta 5_{h 5} \cdot\left(x-x_{3}\right)\right] \cdot d x,
$$




$$
d 5_{h 5}^{x}=\left.\frac{2}{\tau_{x 5}} \cdot \int_{x_{3}}^{x_{3}+\tau_{x 5}} \beta 5_{h 5} \cdot A_{z 2}\right|_{y=y_{3}} \cdot \sin \left[\beta 5_{h 5} \cdot\left(x-x_{3}\right)\right] \cdot d x .
$$

The coefficient $e 5_{n 5}^{y}$ and $f 5_{n 5}^{y}$ are respectively determined using a Fourier series expansion of $\left.A_{z 6}\right|_{x=x_{3} \wedge \forall y}$ and $\left.A_{z 7}\right|_{x=x_{4} \wedge \forall y}$ (see Figure 4e) over the interval $y=\left[y_{2}, y_{3}\right]=\left[y_{2}, y_{2}+\tau_{y 5}\right]$ :

$$
\begin{aligned}
& e 5_{n 5}^{y}=\left.\frac{2}{\tau_{y 5}} \cdot \int_{y_{2}}^{y_{2}+\tau_{y 5}} \lambda 5_{n 5} \cdot A_{z 6}\right|_{x=x_{3}} \cdot \sin \left[\lambda 5_{n 5} \cdot\left(y-y_{2}\right)\right] \cdot d y, \\
& f 5_{n 5}^{y}=\left.\frac{2}{\tau_{y 5}} \cdot \int_{y_{2}}^{y_{2}+\tau_{y 5}} \lambda 5_{n 5} \cdot A_{z 7}\right|_{x=x_{4}} \cdot \sin \left[\lambda 5_{n 5} \cdot\left(y-y_{2}\right)\right] \cdot d y .
\end{aligned}
$$

The expression of $c 5_{h 5}^{x}, d 5_{h 5}^{x}, e 5_{n 5}^{y}$ and $f 5_{n 5}^{y}$ are developed in Appendix $C$.

\subsubsection{Region 6}

According to case-study no 2 (i.e., $B_{y}$ and $A_{z}$ are respectively imposed on $x$ - and $y$-edges of a region) in Appendix $\mathrm{B}$, the magnetic vector potential $A_{z 6}$, which is a solution of (2) satisfying the boundary conditions of Figure 4f, is defined by

$$
\begin{gathered}
A_{z 6}=A_{z 6}^{x}+A_{z 6}^{y}+A_{z P 6}, \\
A_{z 6}^{x}=\mid \begin{array}{l}
\left(y_{3}-y\right) \cdot c 6_{0}^{x}+\left(y-y_{2}\right) \cdot d 6_{0}^{x} \\
\cdots+\sum_{h 6=1}^{\infty}\left\{\frac{c 6_{h 6}^{x}}{\beta 6_{h 6}} \cdot \frac{\operatorname{sh}\left[\beta 6_{h 6} \cdot\left(y_{3}-y\right)\right]}{\operatorname{sh}\left(\beta 6_{h 6} \cdot \tau_{y 6}\right)}+\frac{d 6_{h 6}^{x}}{\beta 6_{h 6}} \cdot \frac{\operatorname{sh}\left[\beta 6_{h 6} \cdot\left(y-y_{2}\right)\right]}{\operatorname{sh}\left(\beta 6_{h 6} \cdot \tau_{y 6}\right)}\right\} \cdot \cos \left[\beta 6_{h 6} \cdot\left(x-x_{2}\right)\right]
\end{array} \\
A_{z 6}^{y}=-\sum_{n 6=1}^{\infty}\left\{\frac{e 6_{n 6}^{y}}{\lambda 6_{n 6}} \cdot \frac{\operatorname{ch}\left[\lambda 6_{n 6} \cdot\left(x-x_{2}\right)\right]}{\operatorname{sh}\left(\lambda 6_{n 6} \cdot \tau_{x 6}\right)}-\frac{f 6_{n 6}^{y}}{\lambda 6_{n 6}} \cdot \frac{\operatorname{ch}\left[\lambda 6_{n 6} \cdot\left(x_{3}-x\right)\right]}{\operatorname{sh}\left(\lambda 6_{n 6} \cdot \tau_{x 6}\right)}\right\} \cdot \sin \left[\lambda 6_{n 6} \cdot\left(y-y_{2}\right)\right] .
\end{gathered}
$$

Considering (27b) and (27c) as well as the form of the current density distribution, i.e., ( $2 \mathrm{~b})$, a particular solution $A_{z P 6}$ can be found. The following quadratic form can be proposed as a particular solution:

$$
A_{z P 6}=-\frac{1}{2} \cdot \mu_{6} \cdot J_{z 6} \cdot y^{2}
$$

The $x$-component of $\vec{B}_{6}$ is defined by

$$
\begin{aligned}
& B_{x 6}=B_{x 6}^{x}+B_{x 6}^{y}+B_{x P 6} \\
& B_{x 6}^{x}=\mid \begin{array}{l}
-c 6_{0}^{x}+d 6_{0}^{x} \\
\cdots+\sum_{h 6=1}^{\infty}\left\{-c 6_{h 6}^{x} \cdot \frac{c h\left[\beta 6_{h 6} \cdot\left(y_{3}-y\right)\right]}{\operatorname{sh}\left(\beta 6_{h 6} \cdot \tau_{y 6}\right)}+d 6_{h 6}^{x} \cdot \frac{\operatorname{ch}\left[\beta 6_{h 6} \cdot\left(y-y_{2}\right)\right]}{\operatorname{sh}\left(\beta 6_{h 6} \cdot \tau_{y 6}\right)}\right\} \cdot \cos \left[\beta 6_{h 6} \cdot\left(x-x_{2}\right)\right],
\end{array} \\
& B_{x 6}^{y}=-\sum_{n 6=1}^{\infty}\left\{e 6_{n 6}^{y} \cdot \frac{\operatorname{ch}\left[\lambda 6_{n 6} \cdot\left(x-x_{2}\right)\right]}{\operatorname{sh}\left(\lambda 6_{n 6} \cdot \tau_{x 6}\right)}-f 6_{n 6}^{y} \cdot \frac{\operatorname{ch}\left[\lambda 6_{n 6} \cdot\left(x_{3}-x\right)\right]}{\operatorname{sh}\left(\lambda 6_{n 6} \cdot \tau_{x 6}\right)}\right\} \cdot \cos \left[\lambda 6_{n 6} \cdot\left(y-y_{2}\right)\right] \text {, } \\
& B_{x P 6}=\frac{\partial A_{z P 6}}{\partial y}=-\mu_{6} \cdot J_{z 6} \cdot y,
\end{aligned}
$$

and the $y$-component of $\vec{B}_{6}$ by

$$
B_{y 6}=B_{y 6}^{x}+B_{y 6}^{y}+B_{y P 6}
$$




$$
\begin{gathered}
B_{y 6}^{x}=\sum_{h 6=1}^{\infty}\left\{c 6_{h 6}^{x} \cdot \frac{\operatorname{sh}\left[\beta 6_{h 6} \cdot\left(y_{3}-y\right)\right]}{\operatorname{sh}\left(\beta 6_{h 6} \cdot \tau_{y 6}\right)}+d 6_{h 6}^{x} \cdot \frac{\operatorname{sh}\left[\beta 6_{h 6} \cdot\left(y-y_{2}\right)\right]}{\operatorname{sh}\left(\beta 6_{h 6} \cdot \tau_{y 6}\right)}\right\} \cdot \sin \left[\beta 6_{h 6} \cdot\left(x-x_{2}\right)\right], \\
B_{y 6}^{y}=\sum_{n 6=1}^{\infty}\left\{e 6_{n 6}^{y} \cdot \frac{\operatorname{sh}\left[\lambda 6_{n 6} \cdot\left(x-x_{2}\right)\right]}{\operatorname{sh}\left(\lambda 6_{n 6} \cdot \tau_{x 6}\right)}+f 6_{n 6}^{y} \cdot \frac{\operatorname{sh}\left[\lambda 6_{n 6} \cdot\left(x_{3}-x\right)\right]}{\operatorname{sh}\left(\lambda 6_{n 6} \cdot \tau_{x 6}\right)}\right\} \cdot \sin \left[\lambda 6_{n 6} \cdot\left(y-y_{2}\right)\right], \\
B_{y P 6}=-\frac{\partial A_{z P 6}}{\partial x}=0,
\end{gathered}
$$

where $h 6$ and $n 6$ are the spatial harmonic orders in Region $6 ; c 6_{0}^{x}, d 6_{0}^{x}, c 6_{h 6^{\prime}}^{x}, d 6_{h 6^{\prime}}^{x} e 6_{n 6}^{y}$ and $f 6_{n 6}^{y}$ the integration constants; $\beta 6_{h 6}=h 6 \cdot \pi / \tau_{x 6}$ with $\tau_{x 6}=x_{3}-x_{2}$; and $\lambda 6_{n 6}=n 6 \cdot \pi / \tau_{y 6}$ with $\tau_{y 6}=y_{3}-y_{2}$.

The coefficients $c \sigma_{0}^{x} \& c 6_{h 6}^{x}$ and $d \sigma_{0}^{x} \& d 6_{h 6}^{x}$ are respectively determined using Fourier series expansion of $\left.A_{z 1}\right|_{\forall x \wedge y=y_{2}}$ and $\left.A_{z 2}\right|_{\forall x \wedge y=y_{3}}$ (see Figure 4f) over the interval $x=\left[x_{2}, x_{3}\right]=\left[x_{2}, x_{2}+\tau_{x 6}\right]$ :

$$
\begin{gathered}
c 6_{0}^{x}=\frac{1}{\tau_{x 6}} \cdot \int_{x_{2}}^{x_{2}+\tau_{x 6}} \frac{1}{\tau_{y 6}} \cdot\left[\left.A_{z 1}\right|_{y=y_{2}}-\left.A_{z P 6}\right|_{y=y_{2}}\right] \cdot d x, \\
c 6_{h 6}^{x}=\frac{2}{\tau_{x 6}} \cdot \int_{x_{2}}^{x_{2}+\tau_{x 6}} \beta 6_{h 6} \cdot\left[\left.A_{z 1}\right|_{y=y_{2}}-\left.A_{z P 6}\right|_{y=y_{2}}\right] \cdot \cos \left[\beta 6_{h 6} \cdot\left(x-x_{2}\right)\right] \cdot d x, \\
d 6_{0}^{x}=\frac{1}{\tau_{x 6}} \cdot \int_{x_{2}}^{x_{2}+\tau_{x 6}} \frac{1}{\tau_{y 6}} \cdot\left[\left.A_{z 2}\right|_{y=y_{3}}-\left.A_{z P 6}\right|_{y=y_{3}}\right] \cdot d x, \\
d 6_{h 6}^{x}=\frac{2}{\tau_{x 6}} \cdot \int_{x_{2}}^{x_{2}+\tau_{x 6}} \beta 6_{h 6} \cdot\left[\left.A_{z 2}\right|_{y=y_{3}}-\left.A_{z P 6}\right|_{y=y_{3}}\right] \cdot \cos \left[\beta 6_{h 6} \cdot\left(x-x_{2}\right)\right] \cdot d x .
\end{gathered}
$$

The coefficient $e 6_{n 6}^{y}$ and $f 6_{n 6}^{y}$ are respectively determined using a Fourier series expansion of $\mu_{6} / \mu_{5}$. $\left.B_{y 5}\right|_{x=x_{3} \wedge \forall y}$ and $\mu_{6} /\left.\mu_{3} \cdot B_{y 3}\right|_{x=x_{2} \wedge \forall y}$ (see Figure 4f) over the interval $y=\left[y_{2}, y_{3}\right]=\left[y_{2}, y_{2}+\tau_{y 6}\right]$ :

$$
\begin{aligned}
& e 6_{n 6}^{y}=\frac{2}{\tau_{y 6}} \cdot \int_{y_{2}}^{y_{2}+\tau_{y 6}}\left[\left.\frac{\mu_{6}}{\mu_{5}} \cdot B_{y 5}\right|_{x=x_{3}}-\left.B_{y P 6}\right|_{x=x_{3}}\right] \cdot \sin \left[\lambda 6_{n 6} \cdot\left(y-y_{2}\right)\right] \cdot d y, \\
& f 6_{n 6}^{y}=\frac{2}{\tau_{y 6}} \cdot \int_{y_{2}}^{y_{2}+\tau_{y 6}}\left[\left.\frac{\mu_{6}}{\mu_{3}} \cdot B_{y 3}\right|_{x=x_{2}}-\left.B_{y P 6}\right|_{x=x_{2}}\right] \cdot \sin \left[\lambda 6_{n 6} \cdot\left(y-y_{2}\right)\right] \cdot d y .
\end{aligned}
$$

The expression of $c 6_{0}^{x}, d 6_{0}^{x}, c 6_{h 6^{\prime}}^{x} d 6_{h 6}^{x}, e 6_{n 6}^{y}$ and $f 6_{n 6}^{y}$ are developed in Appendix C.

\subsubsection{Region 7}

According to case-study no 2 (i.e., $B_{y}$ and $A_{z}$ are respectively imposed on $x$ - and $y$-edges of a region) in Appendix $\mathrm{B}$, the magnetic vector potential $A_{z 7}$, which is a solution of (2) satisfying the boundary conditions of Figure $4 \mathrm{~g}$, is defined by

$$
A_{z 7}=A_{z 7}^{x}+A_{z 7}^{y}+A_{z P 7},
$$

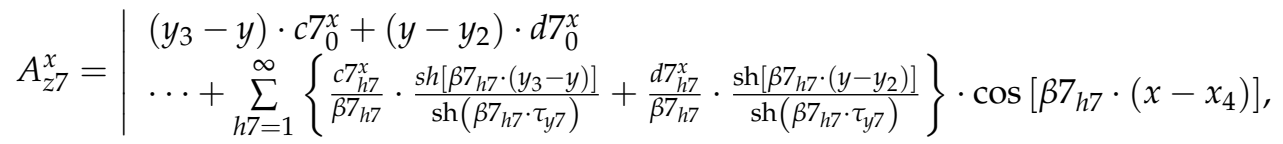




$$
A_{z 7}^{y}=-\sum_{n 7=1}^{\infty}\left\{\frac{e 7_{n 7}^{y}}{\lambda 7_{n 7}} \cdot \frac{\operatorname{ch}\left[\lambda 7_{n 7} \cdot\left(x-x_{4}\right)\right]}{\operatorname{sh}\left(\lambda 7_{n 7} \cdot \tau_{x 7}\right)}-\frac{f 7_{n 7}^{y}}{\lambda 7_{n 7}} \cdot \frac{\operatorname{ch}\left[\lambda 7_{n 7} \cdot\left(x_{5}-x\right)\right]}{\operatorname{sh}\left(\lambda 7_{n 7} \cdot \tau_{x 7}\right)}\right\} \cdot \sin \left[\lambda 7_{n 7} \cdot\left(y-y_{2}\right)\right]
$$

Considering (32b) and (32c) as well as the form of the current density distribution, i.e., (2b), a particular solution $A_{z P 7}$ can be found. The following quadratic form can be proposed as a particular solution:

$$
A_{z P 7}=-\frac{1}{2} \cdot \mu_{7} \cdot J_{z 7} \cdot y^{2}
$$

The $x$-component of $\vec{B}_{7}$ is defined by

$$
\begin{aligned}
& B_{x 7}=B_{x 7}^{x}+B_{x 7}^{y}+B_{x P 7} \\
& B_{x 7}^{x}=\mid \begin{array}{l}
-c 7_{0}^{x}+d 7_{0}^{x} \\
\cdots+\sum_{h 7=1}^{\infty}\left\{-c 7_{h 7}^{x} \cdot \frac{c h\left[\beta 7_{h 7} \cdot\left(y_{3}-y\right)\right]}{\operatorname{sh}\left(\beta 7_{h 7} \cdot \tau_{y 7}\right)}+d 7_{h 7}^{x} \cdot \frac{\operatorname{ch}\left[\beta 7_{h 7} \cdot\left(y-y_{2}\right)\right]}{\operatorname{sh}\left(\beta 7_{h 7} \cdot \tau_{y 7}\right)}\right\} \cdot \cos \left[\beta 7_{h 7} \cdot\left(x-x_{4}\right)\right],
\end{array} \\
& B_{x 7}^{y}=-\sum_{n 7=1}^{\infty}\left\{e 7_{n 7}^{y} \cdot \frac{\operatorname{ch}\left[\lambda 7_{n 7} \cdot\left(x-x_{4}\right)\right]}{\operatorname{sh}\left(\lambda 7_{n 7} \cdot \tau_{x 7}\right)}-f 6_{n 7}^{y} \cdot \frac{\operatorname{ch}\left[\lambda 7_{n 7} \cdot\left(x_{5}-x\right)\right]}{\operatorname{sh}\left(\lambda 7_{n 7} \cdot \tau_{x 7}\right)}\right\} \cdot \cos \left[\lambda 7_{n 7} \cdot\left(y-y_{2}\right)\right], \\
& B_{x P 7}=\frac{\partial A_{z P 7}}{\partial y}=-\mu_{7} \cdot J_{z 7} \cdot y,
\end{aligned}
$$

and the $y$-component of $\vec{B}_{7}$ by

$$
\begin{gathered}
B_{y 7}=B_{y 7}^{x}+B_{y 7}^{y}+B_{y P 7} \\
B_{y 7}^{x}=\sum_{h 7=1}^{\infty}\left\{c 7_{h 7}^{x} \cdot \frac{s h\left[\beta 7_{h 7} \cdot\left(y_{3}-y\right)\right]}{\operatorname{sh}\left(\beta 7_{h 7} \cdot \tau_{y 7}\right)}+d 7_{h 7}^{x} \cdot \frac{\operatorname{sh}\left[\beta 7_{h 7} \cdot\left(y-y_{2}\right)\right]}{\operatorname{sh}\left(\beta 7_{h 7} \cdot \tau_{y 7}\right)}\right\} \cdot \sin \left[\beta 7_{h 7} \cdot\left(x-x_{4}\right)\right], \\
B_{y 7}^{y}=\sum_{n 7=1}^{\infty}\left\{e 7_{n 7}^{y} \cdot \frac{\operatorname{sh}\left[\lambda 7_{n 7} \cdot\left(x-x_{4}\right)\right]}{s h\left(\lambda 7_{n 7} \cdot \tau_{x 7}\right)}+f 7_{n 7}^{y} \cdot \frac{\operatorname{sh}\left[\lambda 7_{n 7} \cdot\left(x_{5}-x\right)\right]}{\operatorname{sh}\left(\lambda 7_{n 7} \cdot \tau_{x 7}\right)}\right\} \cdot \sin \left[\lambda 7_{n 7} \cdot\left(y-y_{2}\right)\right], \\
B_{y P 7}=-\frac{\partial A_{z P 7}}{\partial x}=0,
\end{gathered}
$$

where $h 7$ and $n 7$ are the spatial harmonic orders in Region $7 ; c 7_{0}^{x}, d 7_{0}^{x}, c 7_{h 7}^{x}, d 7_{h 7}^{x}, e 7_{n 7}^{y}$ and $f 7_{n 7}^{y}$ the integration constants; $\beta 7_{h 7}=h 7 \cdot \pi / \tau_{x 7}$ with $\tau_{x 7}=x_{5}-x_{4} ;$ and $\lambda 7_{n 7}=n 7 \cdot \pi / \tau_{y 7}$ with $\tau_{y 7}=y_{3}-y_{2}$.

The coefficients $c 7_{0}^{x} \& c 7_{h 7}^{x}$ and $d 7_{0}^{x} \& d 7_{h 7}^{x}$ are respectively determined using Fourier series expansion of $\left.A_{z 1}\right|_{\forall x \wedge y=y_{2}}$ and $\left.A_{z 2}\right|_{\forall x \wedge y=y_{3}}$ (see Figure 4g) over the interval $x=\left[x_{4}, x_{5}\right]=\left[x_{4}, x_{4}+\tau_{x 7}\right]$ :

$$
\begin{gathered}
c 7_{0}^{x}=\frac{1}{\tau_{x 7}} \cdot \int_{x_{4}}^{x_{4}+\tau_{x 7}} \frac{1}{\tau_{y 7}} \cdot\left[\left.A_{z 1}\right|_{y=y_{2}}-\left.A_{z P 7}\right|_{y=y_{2}}\right] \cdot d x, \\
c 7_{h 7}^{x}=\frac{2}{\tau_{x 7}} \cdot \int_{x_{4}}^{x_{4}+\tau_{x 7}} \beta 7_{h 7} \cdot\left[\left.A_{z 1}\right|_{y=y_{2}}-\left.A_{z P 7}\right|_{y=y_{2}}\right] \cdot \cos \left[\beta 7_{h 7} \cdot\left(x-x_{4}\right)\right] \cdot d x, \\
d 7_{0}^{x}=\frac{1}{\tau_{x 7}} \cdot \int_{x_{4}}^{x_{4}+\tau_{x 7}} \frac{1}{\tau_{y 7}} \cdot\left[\left.A_{z 2}\right|_{y=y_{3}}-\left.A_{z P 7}\right|_{y=y_{3}}\right] \cdot d x, \\
d 7_{h 7}^{x}=\frac{2}{\tau_{x 7}} \cdot \int_{x_{4}}^{x_{4}+\tau_{x 7}} \beta 7_{h 7} \cdot\left[\left.A_{z 2}\right|_{y=y_{3}}-\left.A_{z P 7}\right|_{y=y_{3}}\right] \cdot \cos \left[\beta 7_{h 7} \cdot\left(x-x_{4}\right)\right] \cdot d x .
\end{gathered}
$$


The coefficient $e 7_{n 7}^{y}$ and $f 7_{n 7}^{y}$ are respectively determined using a Fourier series expansion of $\mu_{7} / \mu_{4}$. $\left.B_{y 4}\right|_{x=x_{5} \wedge \forall y}$ and $\mu_{7} /\left.\mu_{5} \cdot B_{y 5}\right|_{x=x_{4} \wedge \forall y}$ (see Figure $4 \mathrm{~g}$ ) over the interval $y=\left[y_{2}, y_{3}\right]=\left[y_{2}, y_{2}+\tau_{y 7}\right]$ :

$$
\begin{aligned}
& e 7_{n 7}^{y}=\frac{2}{\tau_{y 7}} \cdot \int_{y_{2}}^{y_{2}+\tau_{y 7}}\left[\left.\frac{\mu_{7}}{\mu_{4}} \cdot B_{y 4}\right|_{x=x_{5}}-\left.B_{y P 7}\right|_{x=x_{5}}\right] \cdot \sin \left[\lambda 7_{n 7} \cdot\left(y-y_{2}\right)\right] \cdot d y, \\
& f 7_{n 7}^{y}=\frac{2}{\tau_{y 7}} \cdot \int_{y_{2}}^{y_{2}+\tau_{y 7}}\left[\left.\frac{\mu_{7}}{\mu_{5}} \cdot B_{y 5}\right|_{x=x_{4}}-\left.B_{y P 7}\right|_{x=x_{4}}\right] \cdot \sin \left[\lambda 7_{n 7} \cdot\left(y-y_{2}\right)\right] \cdot d y .
\end{aligned}
$$

The expression of $c 7_{0}^{x}, d 7_{0}^{x}, c 7_{h 7}^{x}, d 7_{h 7}^{x}, e 7_{n 7}^{y}$ and $f 7_{n 7}^{y}$ are developed in Appendix C.

\subsection{Solving of Linear System}

The integration constants can be determined by solving the following linear equations which can be written in matrix form as [68]

$$
[I C]=[B C]^{-1} \cdot[E S]
$$

where $[I C]$ is the integration constants vector (of dimension $X_{\max } \times 1$ ),

$$
\begin{aligned}
& {[I C]=\left[\begin{array}{lllllll}
{[I C 1]} & {[I C 2]} & {[I C 3]} & {[I C 4]} & {[I C 5]} & {[I C 6]} & {[I C 7]}
\end{array}\right]^{T},} \\
& {[I C 1]=\left[d 1_{h 1}^{x}\right],} \\
& {[I C 2]=\left[c 2_{h 2}^{x}\right] \text {, }} \\
& {[I C 3]=\left[\begin{array}{lll}
c 3_{h 3}^{x} & d 3_{h 3}^{x} & f 3_{n 3}^{y}
\end{array}\right] \text {, }} \\
& {[\text { IC4 }]=\left[\begin{array}{lll}
c 4_{h 4}^{x} & d 4_{h 4}^{x} & e 4_{n 4}^{y}
\end{array}\right],} \\
& {[I C 5]=\left[\begin{array}{llll}
c 5_{h 5}^{x} & d 5_{h 5}^{x} & e 5_{n 5}^{y} & f 5_{n 5}^{y}
\end{array}\right],} \\
& {[I C 6]=\left[\begin{array}{llllll}
c 6_{0}^{x} & c 6_{h 6}^{x} & d 6_{0}^{x} & d 6_{h 6}^{x} & e 6_{n 6}^{y} & f 6_{n 6}^{y}
\end{array}\right],} \\
& {[I C 7]=\left[\begin{array}{llllll}
c 7_{0}^{x} & c 7_{h 7}^{x} & d 7_{0}^{x} & d 7_{h 7}^{x} & e 7_{n 7}^{y} & f 7_{n 7}^{y}
\end{array}\right],}
\end{aligned}
$$

$[E S]$ the electromagnetic sources vector (of dimension $X_{\max } \times 1$ ),

$$
\begin{aligned}
& {[E S]=\left[\begin{array}{lllllll}
{[E S 1]} & {[E S 2]} & {[E S 3]} & {[E S 4]} & {[E S 5]} & {[E S 6]} & {[E S 7]}
\end{array}\right]^{T},} \\
& {[E S 1]=\left[E S 16_{h 1}+E S 17_{h 1}\right],} \\
& {[E S 2]=\left[E S 26_{h 2}+E S 27_{h 2}\right] \text {, }} \\
& {[E S 3]=\left[\begin{array}{lll}
0 & 0 & E S 36_{n 3}
\end{array}\right],} \\
& {[E S 4]=\left[\begin{array}{lll}
0 & 0 & E S 47_{n 4}
\end{array}\right],} \\
& {[E S 5]=\left[\begin{array}{cccc}
0 & 0 & E S 56_{n 5} & E S 57_{n 5}
\end{array}\right],} \\
& {[E S 6]=\left[\begin{array}{llllll}
E S 61_{0} & 0 & E S 62_{0} & 0 & 0 & 0
\end{array}\right] \text {, }} \\
& {[E S 7]=\left[\begin{array}{llllll}
E S 71_{0} & 0 & E S 72_{0} & 0 & 0 & 0
\end{array}\right] \text {, }}
\end{aligned}
$$


and $[B C]$ the boundary conditions matrix (of dimension $X_{\max } \times X_{\max }$ )

$$
[B C]=\left[\begin{array}{ccccccc}
{[I]} & 0 & {[B C 13]} & {[B C 14]} & {[B C 15]} & {[B C 16]} & {[B C 17]} \\
0 & {[I]} & {[B C 23]} & {[B C 24]} & {[B C 25]} & {[B C 26]} & {[B C 27]} \\
{[B C 31]} & {[B C 32]} & {[I]} & 0 & 0 & {[B C 36]} & 0 \\
{[B C 41]} & {[B C 42]} & 0 & {[I]} & 0 & 0 & {[B C 47]} \\
{[B C 51]} & {[B C 52]} & 0 & 0 & {[I]} & {[B C 56]} & {[B C 57]} \\
{[B C 61]} & {[B C 62]} & {[B C 63]} & 0 & {[B C 65]} & {[I]} & 0 \\
{[B C 71]} & {[B C 72]} & 0 & {[B C 74]} & {[B C 75]} & 0 & {[I]}
\end{array}\right],
$$

in which $[I]$ is identity matrix, and

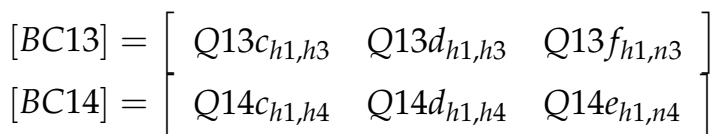

$$
\begin{aligned}
& {[B C 15]=\left[\begin{array}{llll}
Q 15 c_{h 1, h 5} & Q 15 d_{h 1, h 5} & Q 15 e_{h 1, n 5} & Q 15 f_{h 1, n 5}
\end{array}\right]} \\
& {[B C 16]=\left[\begin{array}{llllll}
Q 16 c_{h 1,0} & Q 16 c_{h 1, h 6} & Q 16 d_{h 1,0} & Q 16 d_{h 1, h 6} & Q 16 e_{h 1, n 6} & Q 16 f_{h 1, n 6}
\end{array}\right.}
\end{aligned}
$$

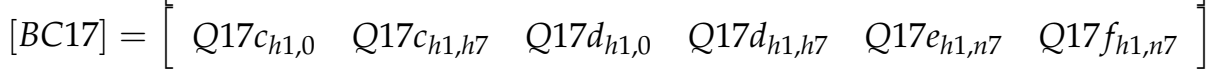

for Region 1,

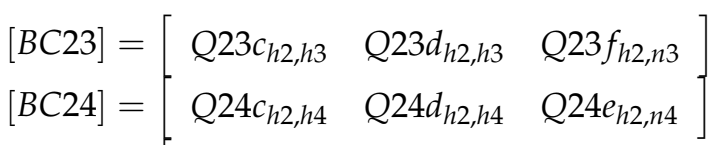

$$
\begin{aligned}
& {[B C 25]=\left[\begin{array}{llll}
Q 25 c_{h 2, h 5} & Q 25 d_{h 2, h 5} & Q 25 e_{h 2, n 5} & Q 25 f_{h 2, n 5}
\end{array}\right]} \\
& {[B C 26]=\left[\begin{array}{llllll}
Q 26 c_{h 2,0} & Q 26 c_{h 2, h 6} & Q 26 d_{h 2,0} & Q 26 d_{h 2, h 6} & Q 26 e_{h 2, n 6} & Q 26 f_{h 2, n 6}
\end{array}\right]} \\
& {[B C 27]=\left[\begin{array}{llllll}
Q 27 c_{h 2,0} & Q 27 c_{h 2, h 7} & Q 27 d_{h 2,0} & Q 27 d_{h 2, h 7} & Q 27 e_{h 2, n 7} & Q 27 f_{h 2, n 7}
\end{array}\right]}
\end{aligned}
$$

for Region 2,

$$
\begin{aligned}
{[B C 31] } & =\left[\begin{array}{lll}
Q 31 d_{h 3, h 1} & 0 & 0
\end{array}\right]^{T} \\
{[B C 32] } & =\left[\begin{array}{cccccc}
0 & Q 32 c_{h 3, h 2} & 0
\end{array}\right]^{T} \\
{[B C 36] } & =\left[\begin{array}{cccccc}
0 & 0 & 0 & 0 & 0 & 0 \\
0 & 0 & 0 & 0 & 0 & 0 \\
Q 36 c_{n 3,0} & Q 36 c_{n 3, h 6} & Q 36 d_{n 3,0} & Q 36 d_{n 3, h 6} & Q 36 e_{n 3, n 6} & Q 36 f_{n 3, n 6}
\end{array}\right]
\end{aligned}
$$

for Region 3,

$$
\begin{aligned}
{[B C 41] } & =\left[\begin{array}{lll}
Q 41 d_{h 4, h 1} & 0 & 0
\end{array}\right]^{T} \\
{[B C 42] } & =\left[\begin{array}{cccccc}
0 & Q 42 c_{h 4, h 2} & 0
\end{array}\right]^{T} \\
{[B C 47] } & =\left[\begin{array}{cccccc}
0 & 0 & 0 & 0 & 0 & 0 \\
0 & 0 & 0 & 0 & 0 & 0 \\
Q 47 c_{n 4,0} & Q 47 c_{n 4, h 7} & Q 47 d_{n 4,0} & Q 47 d_{n 4, h 7} & Q 47 e_{n 4, n 7} & Q 47 f_{n 4, n 7}
\end{array}\right]
\end{aligned}
$$


for Region 4,

$$
\begin{aligned}
& \begin{array}{l}
{[B C 51]=\left[\begin{array}{llll}
Q 51 d_{h 5, h 1} & 0 & 0 & 0
\end{array}\right]^{T}} \\
{[B C 52]=\left[\begin{array}{llll}
0 & Q 52 c_{h 5, h 2} & 0 & 0
\end{array}\right]^{T}}
\end{array} \\
& {[B C 56]=\left[\begin{array}{cccccc}
0 & 0 & 0 & 0 & 0 & 0 \\
0 & 0 & 0 & 0 & 0 & 0 \\
Q 56 c_{n 5,0} & Q 56 c_{n 5, h 6} & Q 56 d_{n 5,0} & Q 56 d_{n 5, h 6} & Q 56 e_{n 5, n 6} & Q 56 f_{n 5, n 6} \\
0 & 0 & 0 & 0 & 0 & 0 \\
0 & 0 & 0 & 0 & 0 & 0 \\
0 & 0 & 0 & 0 & 0 & 0 \\
0 & 0 & 0 & 0 & 0 & 0 \\
Q 57 c_{n 5,0} & Q 57 c_{n 5, h 7} & Q 57 d_{n 5,0} & Q 57 c_{n 5, h 7} & Q 57 e_{n 5, n 7} & Q 57 f_{n 5, n 7}
\end{array}\right]}
\end{aligned}
$$

for Region 5,

$$
\begin{aligned}
& {[B C 61]=\left[\begin{array}{llllll}
Q 61 d_{0, h 1} & Q 61 d_{h 6, h 1} & 0 & 0 & 0 & 0
\end{array}\right]^{T}} \\
& {[B C 62]=\left[\begin{array}{llllll}
0 & 0 & Q 62 c_{0, h 2} & Q 62 c_{h 6, h 2} & 0 & 0
\end{array}\right]^{T}} \\
& {[B C 63]=\left[\begin{array}{ccc}
0 & 0 & 0 \\
0 & 0 & 0 \\
0 & 0 & 0 \\
0 & 0 & 0 \\
0 & 0 & 0 \\
Q 63 c_{n 6, h 3} & Q 63 d_{n 6, h 3} & Q 63 f_{n 6, n 3}
\end{array}\right]} \\
& {[B C 65]=\left[\begin{array}{cccc}
0 & 0 & 0 & 0 \\
0 & 0 & 0 & 0 \\
0 & 0 & 0 & 0 \\
0 & 0 & 0 & 0 \\
Q 65 c_{n 6, h 5} & Q 65 d_{n 6, h 5} & Q 65 e_{n 6, n 5} & Q 65 f_{n 6, n 5} \\
0 & 0 & 0 & 0
\end{array}\right]}
\end{aligned}
$$

for Region 6,

$$
\begin{aligned}
& {[B C 71]=\left[\begin{array}{llllll}
Q 71 d_{0, h 1} & Q 71 d_{h 7, h 1} & 0 & 0 & 0 & 0
\end{array}\right]_{T}^{T}} \\
& {[B C 72]=\left[\begin{array}{llllll}
0 & 0 & Q 72 c_{0, h 2} & Q 72 c_{h 7, h 2} & 0 & 0
\end{array}\right]^{T}} \\
& {[B C 74]=\left[\begin{array}{ccc}
0 & 0 & 0 \\
0 & 0 & 0 \\
0 & 0 & 0 \\
0 & 0 & 0 \\
Q 74 c_{n 7, h 4} & Q 74 d_{n 7, h 4} & Q 74 e_{n 7, n 4} \\
0 & 0 & 0
\end{array}\right]} \\
& {[B C 75]=\left[\begin{array}{cccc}
0 & 0 & 0 & 0 \\
0 & 0 & 0 & 0 \\
0 & 0 & 0 & 0 \\
0 & 0 & 0 & 0 \\
0 & 0 & 0 & 0 \\
Q 75 c_{n 7, h 5} & Q 75 d_{n 7, h 5} & Q 75 e_{n 7, n 5} & Q 75 f_{n 7, n 5}
\end{array}\right]}
\end{aligned}
$$

for Region 7. 
The corresponding elements in (39) and (40) are defined in Appendix C. One can note that (37) consists of

$$
X_{\max }=\left[\begin{array}{l}
H 1_{\max }+H 2_{\max }+2 \cdot H 3_{\max }+N 3_{\max }+2 \cdot H 4_{\max }+N 4_{\max } \\
\cdots+2 \cdot\left(H 5_{\max }+N 5_{\max }\right)+2 \cdot\left(H 6_{\max }+N 6_{\max }+1\right)+2 \cdot\left(H 7_{\max }+N 7_{\max }+1\right)
\end{array}\right]
$$

equations and unknowns. Any mathematical software can quickly give the numerical solution of (37). This set is implemented in Matlab $\mathbb{R}$ (R2015a, Mathworks, Natick, MA, USA) by using the sparse matrix/vectors according to the method described in Section 2. The analytical solutions of $A_{z}$ and $\vec{B}=\left\{B_{x} ; B_{y} ; 0\right\}$ in the various regions have been computed with a finite number of spatial harmonics terms $H 1_{\max }-H 7_{\max }$ (for the $x$-edges) and $N 3_{\max }-N 7_{\max }$ (for the $y$-edges). Usually, the two reasons for the possibility of including a finite number of harmonics is a limiting computational time and numerical accuracy [69].

\subsection{Numerical Problems: Harmonics and Ill-Conditioned System}

A discussion on the numerical limitations of such semi-analytical models has been presented in $[69,70]$. Numerical methods, which use a meshed geometry, will have a limited accuracy related to the density of the mesh. The Maxwell-Fourier methods exhibit a similar problem due to the periodicity of Fourier series, and consequently to the finite number of harmonics.

The size of the model, or more specifically, the size of the matrix $[B C]$, as defined in (40), depends on the number of: (i) subdomains; (ii) boundary conditions; and (iii) spatial harmonics terms. Consequently, an electromagnetic device with a high number of teeth/slots results in large model and, hence, in high computational time [70]. The numerical accuracy of magnetic field solution and the computational time depend on the highest spatial harmonic orders considered in the different subdomains. It is interesting to note that the maximum number of harmonics also depends on the available memory of computer. Beyond a certain number of harmonics, the linear system becomes ill-conditioned and the results inaccurate [69]. Therefore, the number of harmonics has to be carefully selected to obtain a correctly converged solution. An extensive discussion on the effect of the harmonics number taken into account is given in [71,72]. However, owing to the different sizes of the regions (e.g.,the finite height/width, etc.), such series could be truncated at different points. Considering an optimal ratio between the numbers of harmonic terms taken into account in each region might lead to a lower calculation error and a higher rate of convergence [72].

Limiting the number of harmonics will lead to inaccurate field solutions at discontinuous points in the geometry, especially at the corner points of magnets, current regions, or soft-magnetic material [69]. Moreover, the Gibbs phenomenon can become dominant at these positions (at interfaces between region with unequal width) [73].

\section{Comparison of the Semi-Analytic and Finite-Element Calculations}

\subsection{Introduction}

The objective of this section is to show the effectiveness of 2-D subdomain model on the magnetic field distribution. The main parameters of the air- and iron-cored coil are given in Table 1. For the comparison, the system has been set up using Cedrat's Flux2D (Version 10.2.1., Altair Engineering, Meylan Cedex, France) software package (i.e., an advanced finite-element method based numeric field analysis program) [8]. The finite-element computations are done under same assumptions on which the semi-analytical model is based (see § 2.1. Problem Description and Assumptions). The spatial harmonics terms in each subdomain, given in Table 1 (rounded to 0 decimal), have been imposed according to an optinal ratio as indicated in [71,72], i.e., for $H 1_{\max }$ given,

$$
H \bullet_{\max }=H 1_{\max } \cdot \frac{\tau_{x}}{\tau_{x 1}} \text { and } N \bullet_{\max }=H \bullet_{\max } \cdot \frac{\tau_{x}}{\tau_{y \bullet}} .
$$


The linear system (37) consists of 3,346 elements (representing the size of linear system to solve) which is much smaller than the 2-D FEA mesh having 8,566 surfaces elements of second order (viz., the triangles number of system). The 2-D FEA mesh for an air- or iron-cored coil is illustrated in Figure 5. The personal computer used for this comparison has the following characteristics: HP Z800 Intel(R) Xeon(R) CPU@2.4 GHz (with 2 processors) RAM 16 Go 64 bits. The computation time of 2-D subdomain model is equal to $0.01 \mathrm{sec}$ for 2-D subdomain model and $1 \mathrm{sec}$ for the 2-D FEA. The proposed design approach can thus reduce the computation time by approximately 100-fold versus to 2-D FEA.

Table 1. Parameters of the Air- or Iron-cored Coil.

\begin{tabular}{lc}
\hline Parameters, Symbols [Units] & Values \\
\hline Number of coils turns, $N_{t}[-]$ & 1600 \\
Maximum direct current, $I[\mathrm{~A}]$ & 5 \\
Surface of conductors, $S_{c}\left[\mathrm{~mm}{ }^{2}\right]$ & 800 \\
Current density (due to supply currents), $J_{z k}\left[\mathrm{~A} / \mathrm{mm}^{2}\right]$ & \pm 10 \\
Effective axial length, $L_{z}[\mathrm{~cm}]$ & 4 \\
Geometrical parameters in the x-axis, $\left\{x_{1} ; x_{2} ; x_{3} ; x_{4} ; x_{5} ; x_{6}\right\}[\mathrm{cm}]$ & $\{0 ; 10 ; 12 ; 16 ; 18 ; 28\}$ \\
Geometrical parameters in the y-axis, $\left\{y_{1} ; y_{2} ; y_{3} ; y_{4}\right\}[\mathrm{cm}]$ & $\{0 ; 10 ; 14 ; 24\}$ \\
Relative magnetic permeability of the iron, $\mu_{i r o n}[-]$ & 1500 \\
Number of spatial harmonics for Region $1, H 1_{\max }[-]$ & 300 \\
Number of spatial harmonics for Region $2, H 2_{\max }[-]$ & 300 \\
Number of spatial harmonics for Region $3,\left\{H 3_{\max } ; N 3_{\max }\right\}[-]$ & $\{107 ; 268\}$ \\
Number of spatial harmonics for Region $4,\left\{H 4_{\max } ; N 4_{\max }\right\}[-]$ & $\{107 ; 268\}$ \\
Number of spatial harmonics for Region $5,\left\{H 5_{\max } ; N 5_{\max }\right\}[-]$ & $\{43 ; 268\}$ \\
Number of spatial harmonics for Region $6,\left\{H 6_{\max } ; N 6_{\max }\right\}[-]$ & $\{21 ; 268\}$ \\
Number of spatial harmonics for Region $7,\left\{H 7_{\max } ; N 7_{\max }\right\}[-]$ & $\{21 ; 268\}$ \\
\hline
\end{tabular}

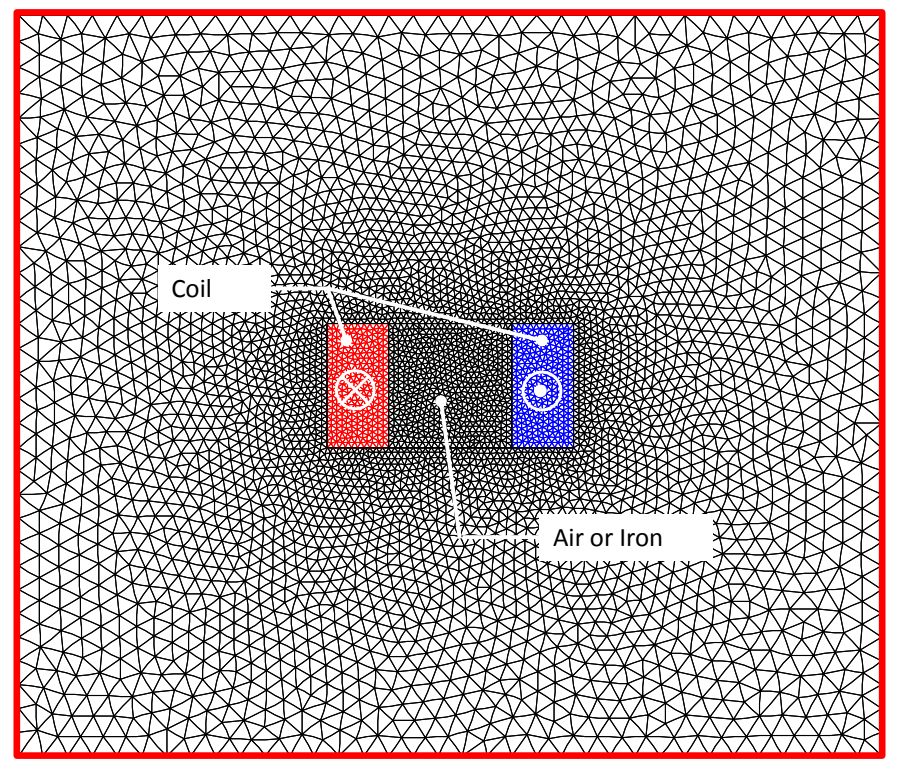

Figure 5. 2-D finite-element analysis (FEA) mesh for an air- or iron-cored coil.

\subsection{Results Discussion}

The 2-D subdomain model is implemented so that it is possible to get values of $A_{z}$ in the airand iron-cored coil. Figure 6 present the equipotential lines ( $\approx 30$ lines) of $A_{z}$ in the system with the 2-D subdomain model and 2-D FEA. As can be seen, a good evaluation is obtained, comparing those results with 2-D FEA, for both air- and iron-core. 

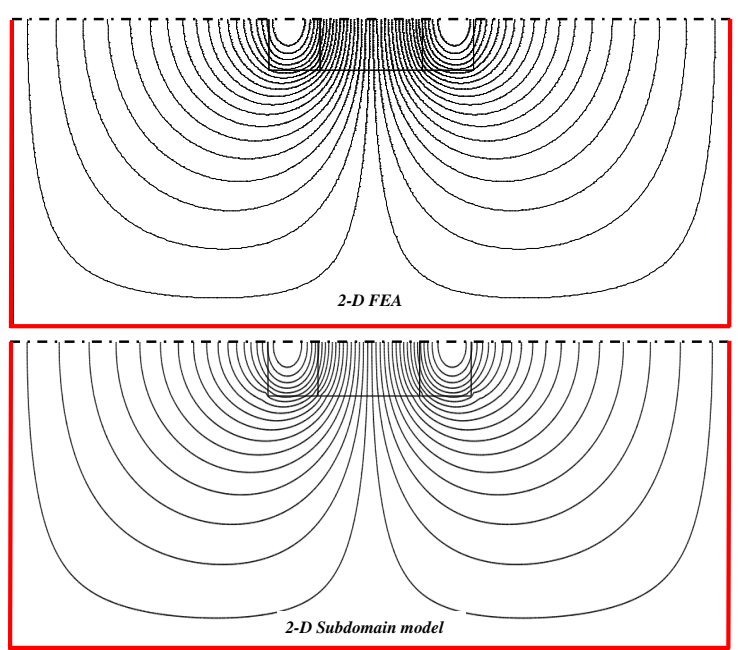

(a)
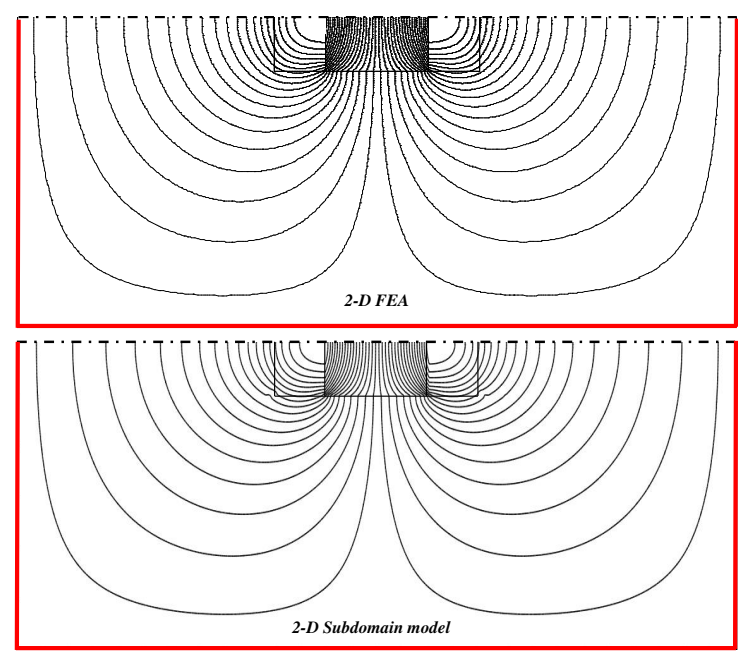

(b)

Figure 6. Equipotential lines of $A_{z}$ with the 2-D subdomain model and FEA for an (a) air- and (b) iron-cored coil.

The paths of the magnetic flux density validation for the comparison are given in Figure 7. The waveforms of the components of $\vec{B}=\left\{B_{x} ; B_{y} ; 0\right\}$ are represented on the various paths in Figures $8-12$. The solid lines represent the magnetic flux density computed by the 2-D FEA and the circles correspond to 2-D subdomain model. It can be seen that a very good agreement is obtained for the components of $\vec{B}$, whatever the paths, for both air- and iron-core. This confirms that the effect of global saturation, with a constant magnetic permeability corresponding to linear zone of $B(H)$ curve, is taken into account accurately. Nevertheless, the numerical accuracy of magnetic field solution is reduced as the number of considered is lowered. The relative error is $\approx 1.5 \%$ for the different components of magnetic flux density. Some slight discrepancies are observed between numerical and analytical results (see Figures 11 and $12 \mathrm{~b}$ ) which can be caused by the finite number of spatial harmonic taken into account in the semi-analytical model according to the $x$ - and $y$-edges (see $\S 2.7$. Numerical Problems: Harmonics and Ill-conditioned System). The increase of harmonics number can resolve these deviations, however, at the expense of the computation time.

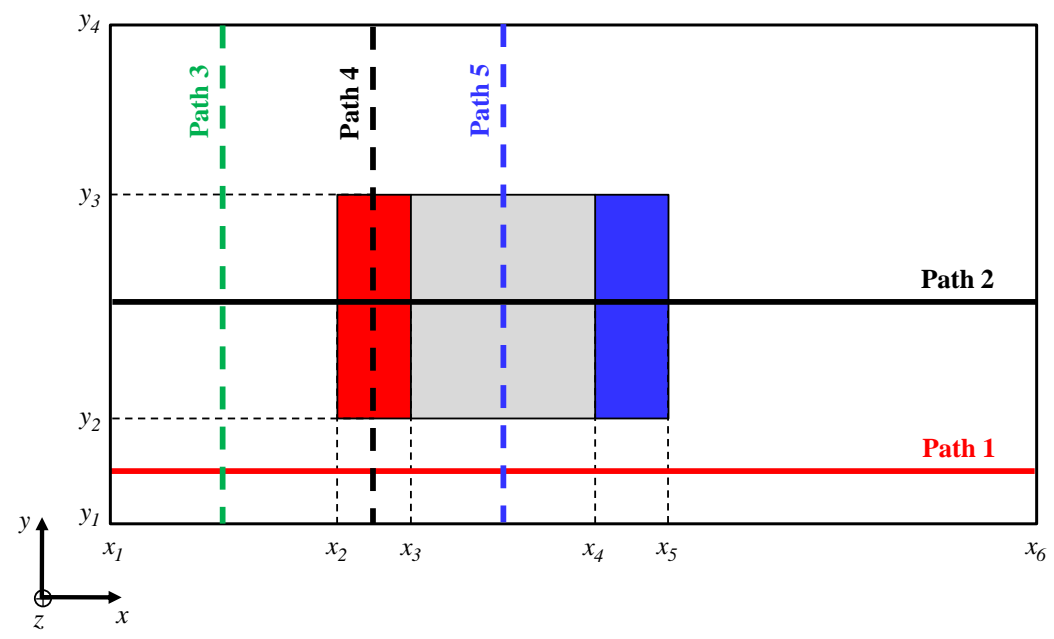

Figure 7. Paths of the magnetic flux density validation for the comparison. 


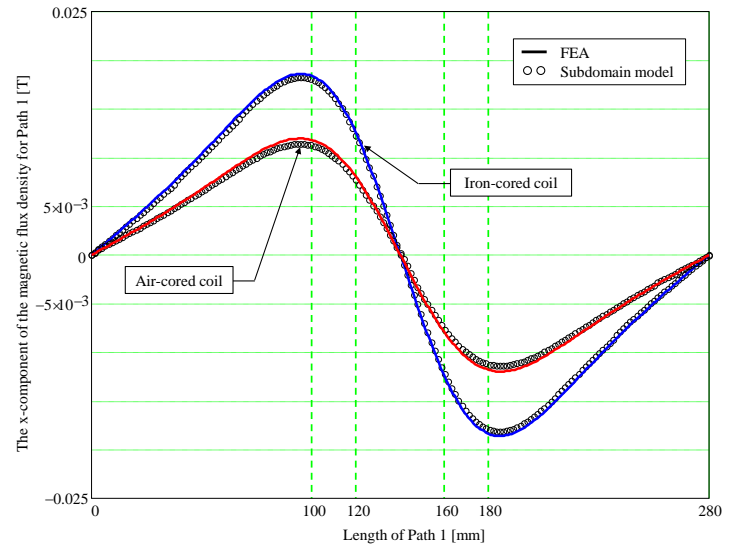

(a)

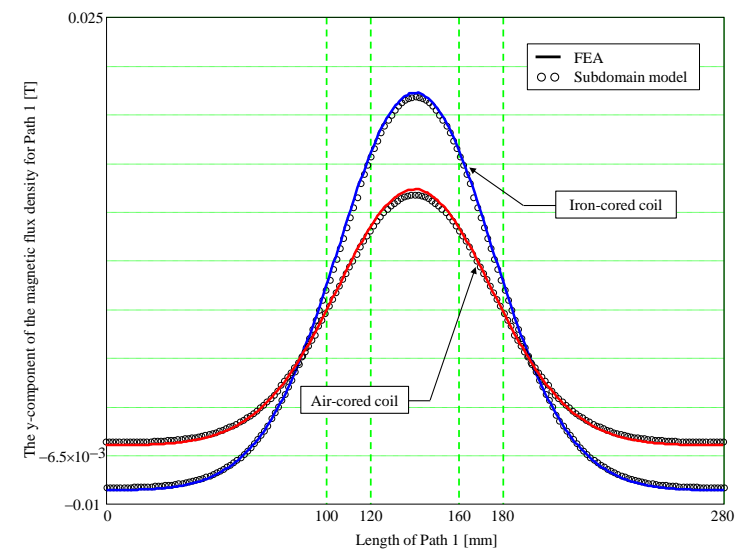

(b)

Figure 8. Waveform of the magnetic flux density for Path 1: (a) $x$-and (b) $y$-component; 2-D FEA.

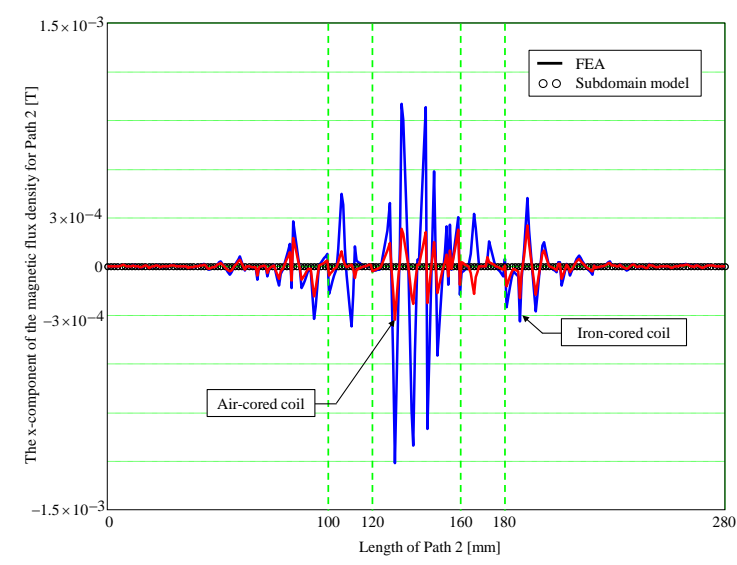

(a)

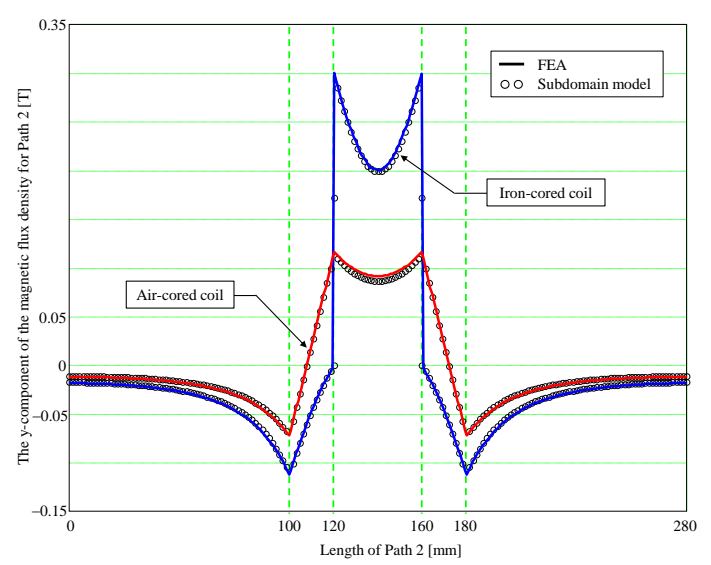

(b)

Figure 9. Waveform of the magnetic flux density for Path 2: (a) $x$ - and (b) $y$-component.

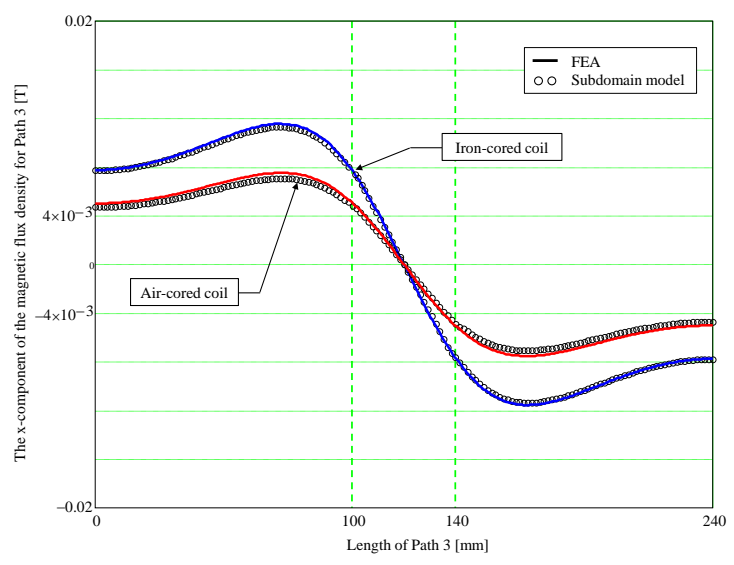

(a)

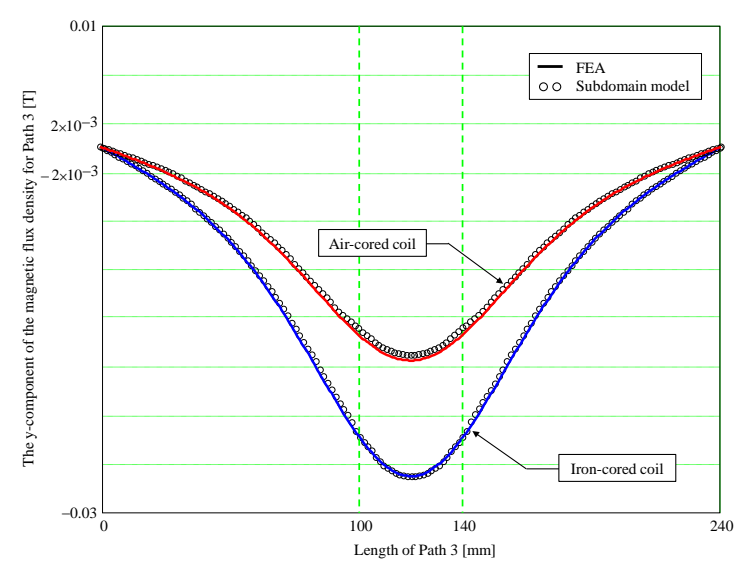

(b)

Figure 10. Waveform of the magnetic flux density for Path 3: (a) $x$ - and (b) $y$-component. 


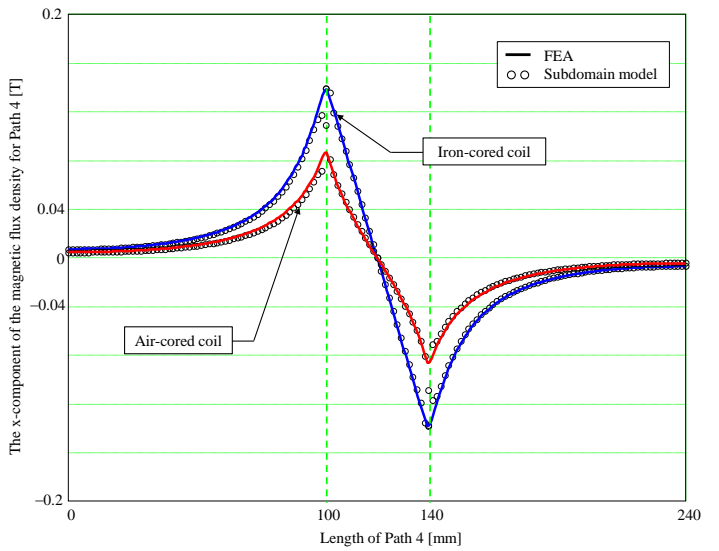

(a)

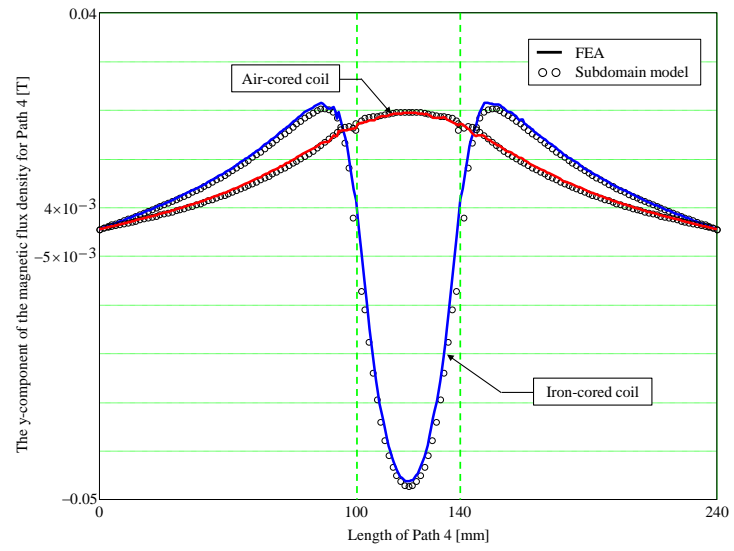

(b)

Figure 11. Waveform of the magnetic flux density for Path 4: (a) $x$-and (b) $y$-component.

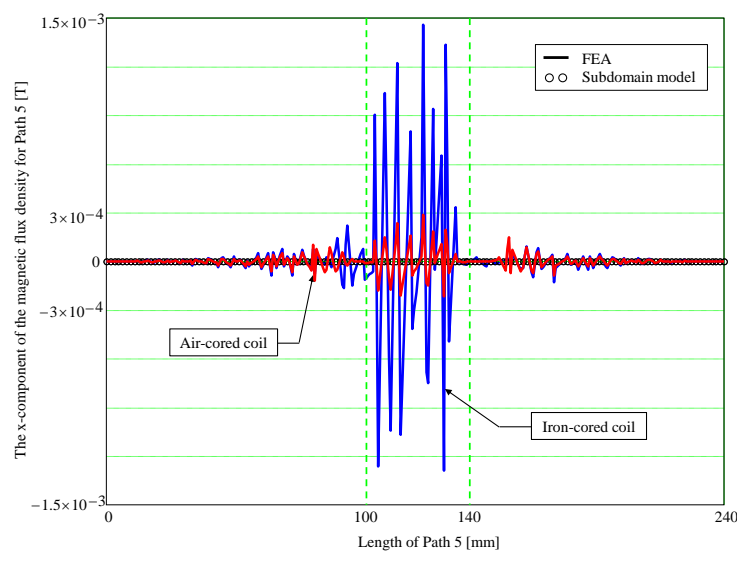

(a)

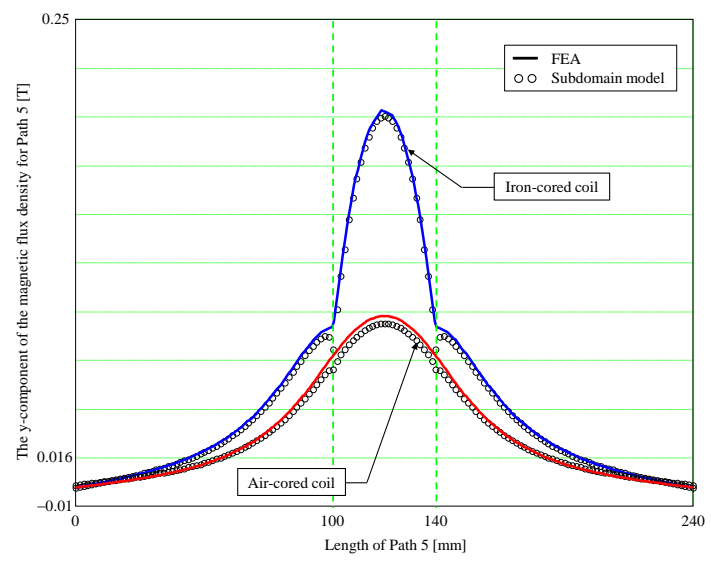

(b)

Figure 12. Waveform of the magnetic flux density for Path 5: (a) $x$ - and (b) $y$-component.

\section{Conclusions}

An overview on the existing (semi-)analytical models in Maxwell-Fourier methods (i.e., multi-layers models and subdomain technique) with the saturation effect has been realized. It has been demonstrated that there is no (semi-)analytical model based on the subdomain technique taking into account the iron parts with(out) the nonlinear $B(H)$ curve. Then, the new scientific contribution on the 2-D subdomain technique in Cartesian coordinates to study the local magnetic field distribution in the iron parts with a global saturation is presented in this paper.

For example, it was performed by solving 2-D magnetostatic Maxwell's equations in Cartesian coordinates $(x, y)$ for an air- or iron-cored coil supplied by a direct current. The subdomains connection is carried out in the two directions (i.e., $x$ - and $y$-edges). The iron magnetic permeability is constant, corresponding to linear zone of the initial magnetization curve. However, nonlinear magnetic materials could be accounted for by means of an iterative algorithm as in [48]. This major scientific contribution will be applied to rotating and/or linear electrical machines with(out) magnets supplied by a direct current or alternate current (with any waveforms) whose the analysis would be based on a 2-D semi-analytical model in Cartesian coordinates (e.g., plane linear machines, axial-flux machines, etc.). An extension of the 2-D subdomain technique in polar coordinates as well as various electrical machines (viz., radial-/axial-/transverse-flux machines, linear machines, U-/E-cored electromagnetic device, etc.) will be made in the next studies. 
This new approach to account for the effect of global saturation is (semi-)analytically based and takes significantly less computing time than the FEA (approximately 100-fold versus to FEA); it is eminently suitable for design and optimization of the electromechanical systems. Predicted results from the exact (semi-)analytical model have been compared finite-element predictions, and good agreement has been achieved, in both amplitudes and waveforms.

Author Contributions: The work presented here was carried out in cooperation among all authors, which have written the paper and have gave advice for the manuscripts.

Conflicts of Interest: The authors declare no conflict of interest.

\section{Appendix A. The 2-D Magnetostatic General Solution in Cartesian Coordinates}

Appendix A.1. Governing Partial Differential Equations (EDPs)

By assuming that the term $\partial \vec{D} / \partial t$ is negligible, the magnetostatic Maxwell's equations are represented by Maxwell-Ampère

$$
\overrightarrow{\operatorname{rot}(\vec{H})}=\vec{J} \quad \text { (with } \vec{J}=0 \text { for the no-load operation), }
$$

and Maxwell-Thomson

$$
\begin{gathered}
\operatorname{div}(\vec{B})=0 \quad \text { (Magnetic flux conservation), } \\
\vec{B}=\overrightarrow{\operatorname{rot}(\vec{A})} \text { with } \operatorname{div}(\vec{A})=0 \quad \text { (Coulomb's gauge), }
\end{gathered}
$$

where $\vec{A}, \vec{B}, \vec{H}$, and $\vec{J}$ are respectively the magnetic vector potential, the magnetic flux density, magnetic field, and the current density (due to supply currents) vectors.

The field vectors $\vec{B}$ and $\vec{H}$ are coupled by the magnetic material equation

$$
\vec{B}=\mu \cdot \vec{H}+\mu_{0} \cdot \vec{M},
$$

where $\vec{M}$ is the magnetization vector (with $\vec{M}=0$ for the vacuum/iron or $\vec{M} \neq 0$ for the magnets according to the magnetization direction [4]), and $\mu=\mu_{0} \cdot \mu_{r}$ the absolute magnetic permeability of the magnetic material in which $\mu_{0}$ and $\mu_{r}$ are respectively the vacuum permeability and the relative permeability of the magnetic material (with $\mu_{r}=1$ for the vacuum or $\mu_{r} \neq 1$ for the magnets/iron).

By using (A1) and (A2), the general EDPs of magnetostatic are defined by [74]:

$$
\begin{gathered}
\vec{X}_{A}-v \cdot \Delta \vec{A}=\vec{J}+\vec{X}_{B}, \\
\vec{X}_{A}=\overrightarrow{\operatorname{grad}(v)} \wedge \overrightarrow{\operatorname{rot}(\vec{A})}, \\
\vec{X}_{B}=\mu_{0} \cdot[\overrightarrow{\operatorname{grad}(v)} \wedge \vec{M}+v \cdot \overrightarrow{\operatorname{rot}(\vec{M})}],
\end{gathered}
$$

where $v=1 / \mu$ is the absolute magnetic reluctivity of the magnetic material.

By neglecting the end-effects (i.e., the system is infinitely long which leads to $\vec{A}=\left\{0 ; 0 ; A_{z}\right\}$ : the magnetic variables are independent of $z$ ), (A3) in Cartesian coordinates $(x, y)$ with $\mu=C^{s t}$ can be expressed by:

$$
\begin{gathered}
\Delta A_{z}=\frac{\partial^{2} A_{z}}{\partial x^{2}}+\frac{\partial^{2} A_{z}}{\partial y^{2}}=E S \\
E S=-\left[\mu \cdot J_{z}+\mu_{0} \cdot\left(\frac{\partial M_{y}}{\partial x}-\frac{\partial M_{x}}{\partial y}\right)\right] .
\end{gathered}
$$


Appendix A.2. General Solution

It is interesting to note that $A_{z}$ is governed by Poisson's equation, when there is one or more electromagnetic sources (i.e., $E S \neq 0$ ), or Laplace's equation, when there is no electromagnetic sources (i.e., $E S=0$ ). According to the method of separation of variables, the 2-D magnetostatic general solution of $A_{z}$ in Cartesian coordinates $(x, y)$ can be written as

$$
\begin{aligned}
& A_{z}=A_{z}^{x}+A_{z}^{y}+A_{z P},
\end{aligned}
$$

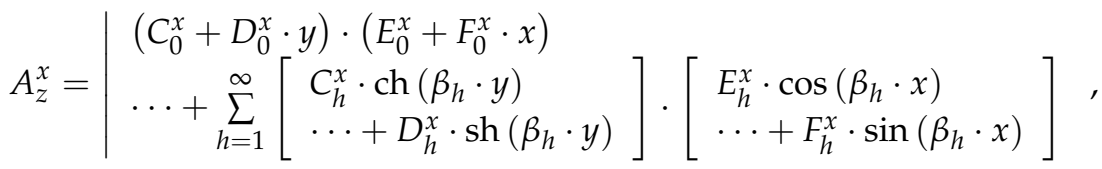

$$
\begin{aligned}
& A_{z}^{y}=\mid \begin{array}{l}
\left(C_{0}^{y}+D_{0}^{y} \cdot y\right) \cdot\left(E_{0}^{y}+F_{0}^{y} \cdot x\right) \\
\cdots+\sum_{n=1}^{\infty}\left[\begin{array}{l}
C_{n}^{y} \cdot \cos \left(\lambda_{n} \cdot y\right) \\
\cdots+D_{n}^{y} \cdot \sin \left(\lambda_{n} \cdot y\right)
\end{array}\right] \cdot\left[\begin{array}{l}
E_{n}^{y} \cdot \operatorname{ch}\left(\lambda_{n} \cdot x\right) \\
\cdots+F_{n}^{y} \cdot \operatorname{sh}\left(\lambda_{n} \cdot x\right)
\end{array}\right],
\end{array}
\end{aligned}
$$

where $A_{z P}$ are the particular solution of $A_{z}$ respecting the second member $E S$ in (A4), $C_{0}^{x}-F_{h}^{x} \& C_{0}^{y}-F_{h}^{y}$ the integration constants, $\beta_{h} \& \lambda_{n}$ the periodicity of $A_{z}^{x} \& A_{z}^{y}$, and $h$ and $n$ the spatial harmonic orders.

According to (A1c), the components of $\vec{B}=\left\{B_{x} ; B_{y} ; 0\right\}$ can be deduced from $A_{z}$ by

$$
B_{x}=\frac{\partial A_{z}}{\partial y} \quad \text { and } \quad B_{y}=-\frac{\partial A_{z}}{\partial x}
$$

which leads to

$$
\begin{aligned}
& B_{x}=B_{x}^{x}+B_{x}^{y}+\frac{\partial A_{z P}}{\partial y}, \\
& B_{x}^{x}=\mid \begin{array}{l}
D_{0}^{x} \cdot\left(E_{0}^{x}+F_{0}^{x} \cdot x\right) \\
\cdots+\sum_{h=1}^{\infty} \beta_{h} \cdot\left[\begin{array}{l}
C_{h}^{x} \cdot \operatorname{sh}\left(\beta_{h} \cdot y\right) \\
\cdots+D_{h}^{x} \cdot \operatorname{ch}\left(\beta_{h} \cdot y\right)
\end{array}\right] \cdot\left[\begin{array}{l}
E_{h}^{x} \cdot \cos \left(\beta_{h} \cdot x\right) \\
\cdots+F_{h}^{x} \cdot \sin \left(\beta_{h} \cdot x\right)
\end{array}\right],
\end{array} \\
& B_{x}^{y}=\mid \begin{array}{l}
D_{0}^{y} \cdot\left(E_{0}^{y}+F_{0}^{y} \cdot x\right) \\
\cdots+\sum_{n=1}^{\infty} \lambda_{n} \cdot\left[\begin{array}{l}
-C_{n}^{y} \cdot \sin \left(\lambda_{n} \cdot y\right) \\
\cdots+D_{n}^{y} \cdot \cos \left(\lambda_{n} \cdot y\right)
\end{array}\right] \cdot\left[\begin{array}{l}
E_{n}^{y} \cdot \operatorname{ch}\left(\lambda_{n} \cdot x\right) \\
\cdots+F_{n}^{y} \cdot \operatorname{sh}\left(\lambda_{n} \cdot x\right)
\end{array}\right],
\end{array}
\end{aligned}
$$

and

$$
\begin{aligned}
& B_{y}=B_{y}^{x}+B_{y}^{y}-\frac{\partial A_{z P}}{\partial x}, \\
& B_{y}^{x}=-\mid \begin{array}{l}
F_{0}^{x} \cdot\left(C_{0}^{x}+D_{0}^{x} \cdot y\right) \\
\cdots+\sum_{h=1}^{\infty} \beta_{h} \cdot\left[\begin{array}{l}
C_{h}^{x} \cdot \operatorname{ch}\left(\beta_{h} \cdot y\right) \\
\cdots+D_{h}^{x} \cdot \operatorname{sh}\left(\beta_{h} \cdot y\right)
\end{array}\right] \cdot\left[\begin{array}{l}
-E_{h}^{x} \cdot \sin \left(\beta_{h} \cdot x\right) \\
\cdots+F_{h}^{x} \cdot \cos \left(\beta_{h} \cdot x\right)
\end{array}\right],
\end{array} \\
& B_{y}^{y}=-\mid \begin{array}{l}
F_{0}^{y} \cdot\left(C_{0}^{y}+D_{0}^{y} \cdot y\right) \\
\cdots+\sum_{n=1}^{\infty} \lambda_{n} \cdot\left[\begin{array}{l}
C_{n}^{y} \cdot \cos \left(\lambda_{n} \cdot y\right) \\
\cdots+D_{n}^{y} \cdot \sin \left(\lambda_{n} \cdot y\right)
\end{array}\right] \cdot\left[\begin{array}{l}
E_{n}^{y} \cdot \operatorname{sh}\left(\lambda_{n} \cdot x\right) \\
\cdots+F_{n}^{y} \cdot \operatorname{ch}\left(\lambda_{n} \cdot x\right)
\end{array}\right] .
\end{array}
\end{aligned}
$$

\section{Appendix B. Simplification of Laplace's Equations According to Imposed Boundary Conditions}

\section{Appendix B.1. Case-Study No 1: $A_{z}$ Imposed on All Edges of a Region}

Figure B1a shows a region (for $x \in\left[x_{l}, x_{r}\right]$ and $y \in\left[y_{l}, y_{t}\right]$ ) whose the magnetic vector potentials are imposed on all edges. By applying the principle of superposition on the magnetic quantities, Figure B1a is redefined by Figure B1b. 


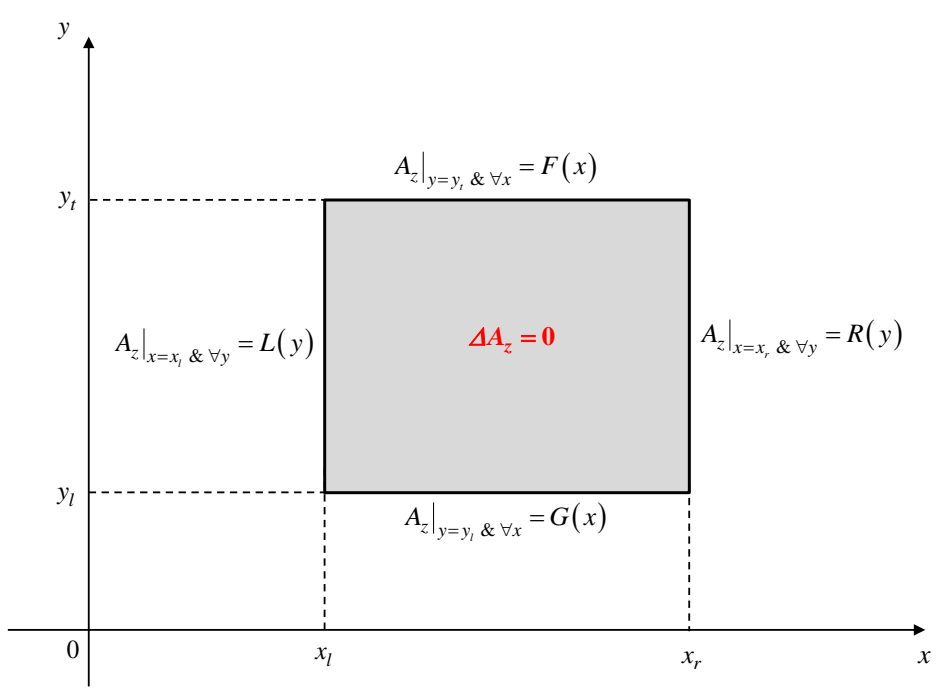

(a)

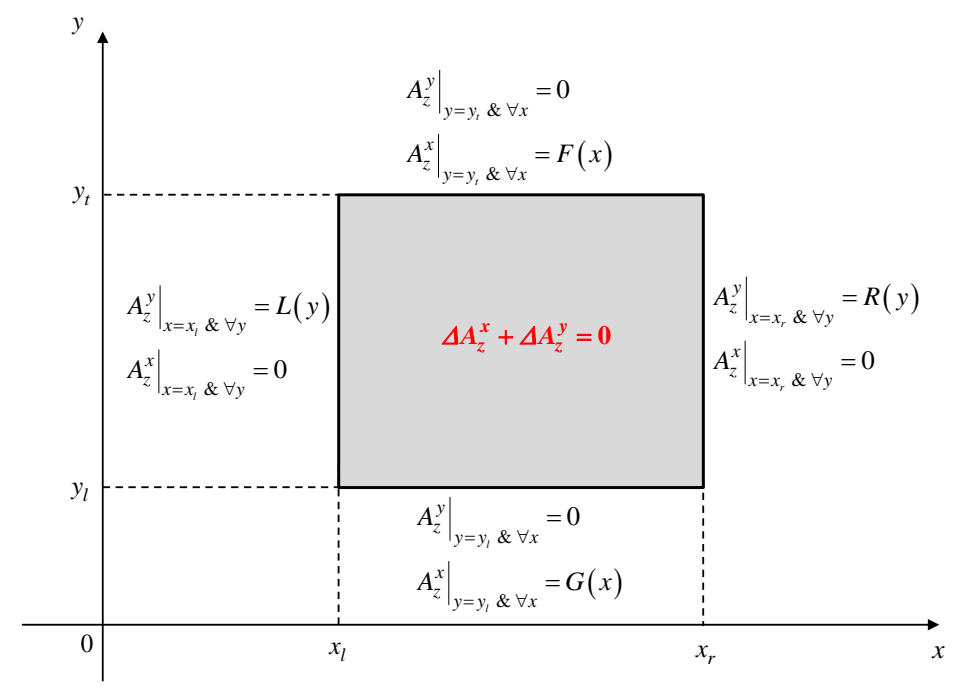

(b)

Figure B1. $A_{z}$ imposed on all edges of a region: (a) General and (b) Principle of superposition.

In the case-study no 1 , the magnetic vector potential $A_{z}=A_{z}^{x}+A_{z}^{y}$, i.e., (A5), is redefined by

$$
\begin{aligned}
& A_{z}^{x}=\sum_{h=1}^{\infty}\left\{\frac{c_{h}^{x}}{\beta_{h}} \cdot \frac{\operatorname{sh}\left[\beta_{h} \cdot\left(y_{t}-y\right)\right]}{\operatorname{sh}\left(\beta_{h} \cdot \tau_{y}\right)}+\frac{d_{h}^{x}}{\beta_{h}} \cdot \frac{\operatorname{sh}\left[\beta_{h} \cdot\left(y-y_{l}\right)\right]}{\operatorname{sh}\left(\beta_{h} \cdot \tau_{y}\right)}\right\} \cdot \sin \left[\beta_{h} \cdot\left(x-x_{l}\right)\right], \\
& A_{z}^{y}=\sum_{n=1}^{\infty}\left\{\frac{e_{n}^{y}}{\lambda_{n}} \cdot \frac{\operatorname{sh}\left[\lambda_{n} \cdot\left(x_{r}-x\right)\right]}{\operatorname{sh}\left(\lambda_{n} \cdot \tau_{x}\right)}+\frac{f_{n}^{y}}{\lambda_{n}} \cdot \frac{\operatorname{sh}\left[\lambda_{n} \cdot\left(x-x_{l}\right)\right]}{\operatorname{sh}\left(\lambda_{n} \cdot \tau_{x}\right)}\right\} \cdot \sin \left[\lambda_{n} \cdot\left(y-y_{l}\right)\right],
\end{aligned}
$$

the component $B_{x}=B_{x}^{x}+B_{x}^{y}$ of $\vec{B}$, i.e., (A7), by

$$
\begin{aligned}
& B_{x}^{x}=\sum_{h=1}^{\infty}\left\{-c_{h}^{x} \cdot \frac{\operatorname{ch}\left[\beta_{h} \cdot\left(y_{t}-y\right)\right]}{\operatorname{sh}\left(\beta_{h} \cdot \tau_{y}\right)}+d_{h}^{x} \cdot \frac{\operatorname{ch}\left[\beta_{h} \cdot\left(y-y_{l}\right)\right]}{\operatorname{sh}\left(\beta_{h} \cdot \tau_{y}\right)}\right\} \cdot \sin \left[\beta_{h} \cdot\left(x-x_{l}\right)\right], \\
& B_{x}^{y}=\sum_{n=1}^{\infty}\left\{e_{n}^{y} \cdot \frac{\operatorname{sh}\left[\lambda_{n} \cdot\left(x_{r}-x\right)\right]}{\operatorname{sh}\left(\lambda_{n} \cdot \tau_{x}\right)}+f_{n}^{y} \cdot \frac{\operatorname{sh}\left[\lambda_{n} \cdot\left(x-x_{l}\right)\right]}{\operatorname{sh}\left(\lambda_{n} \cdot \tau_{x}\right)}\right\} \cdot \cos \left[\lambda_{n} \cdot\left(y-y_{l}\right)\right],
\end{aligned}
$$


and the component $B_{y}=B_{y}^{x}+B_{y}^{y}$ of $\vec{B}$, i.e., (A8), by

$$
\begin{aligned}
& B_{y}^{x}=-\sum_{h=1}^{\infty}\left\{c_{h}^{x} \cdot \frac{\operatorname{sh}\left[\beta_{h} \cdot\left(y_{t}-y\right)\right]}{\operatorname{sh}\left(\beta_{h} \cdot \tau_{y}\right)}+d_{h}^{x} \cdot \frac{\operatorname{sh}\left[\beta_{h} \cdot\left(y-y_{l}\right)\right]}{\operatorname{sh}\left(\beta_{h} \cdot \tau_{y}\right)}\right\} \cdot \cos \left[\beta_{h} \cdot\left(x-x_{l}\right)\right], \\
& B_{y}^{y}=-\sum_{n=1}^{\infty}\left\{-e_{n}^{y} \cdot \frac{\operatorname{ch}\left[\lambda_{n} \cdot\left(x_{r}-x\right)\right]}{\operatorname{sh}\left(\lambda_{n} \cdot \tau_{x}\right)}+f_{n}^{y} \cdot \frac{\operatorname{ch}\left[\lambda_{n} \cdot\left(x-x_{l}\right)\right]}{\operatorname{sh}\left(\lambda_{n} \cdot \tau_{x}\right)}\right\} \cdot \sin \left[\lambda_{n} \cdot\left(y-y_{l}\right)\right],
\end{aligned}
$$

where $c_{h}^{x}, d_{h}^{x}, e_{n}^{y}$ and $f_{n}^{y}$ are new integration constants; $\beta_{h}=h \cdot \pi / \tau_{x}$ with $\tau_{x}=x_{r}-x_{l}$; and $\lambda_{n}=$ $n \cdot \pi / \tau_{y}$ with $\tau_{y}=y_{t}-y_{l}$.

For the particular case illustrated in Figure B2 (whose the magnetic vector potentials are zero on $x$-edges and imposed on $y$-edges), the magnetic vector potential $A_{z}$, according to (B1) with $A_{z}^{y}=0$, is expressed by

$$
A_{z}=\sum_{h=1}^{\infty}\left\{\frac{c_{h}^{x}}{\beta_{h}} \cdot \frac{\operatorname{sh}\left[\beta_{h} \cdot\left(y_{t}-y\right)\right]}{\operatorname{sh}\left(\beta_{h} \cdot \tau_{y}\right)}+\frac{d_{h}^{x}}{\beta_{h}} \cdot \frac{\operatorname{sh}\left[\beta_{h} \cdot\left(y-y_{l}\right)\right]}{\operatorname{sh}\left(\beta_{h} \cdot \tau_{y}\right)}\right\} \cdot \sin \left[\beta_{h} \cdot\left(x-x_{l}\right)\right],
$$

the $x$-component of $\vec{B}$, according to (B6) with $B_{x}^{y}=0$, by

$$
B_{x}=\sum_{h=1}^{\infty}\left\{-c_{h}^{x} \cdot \frac{\operatorname{ch}\left[\beta_{h} \cdot\left(y_{t}-y\right)\right]}{\operatorname{sh}\left(\beta_{h} \cdot \tau_{y}\right)}+d_{h}^{x} \cdot \frac{\operatorname{ch}\left[\beta_{h} \cdot\left(y-y_{l}\right)\right]}{\operatorname{sh}\left(\beta_{h} \cdot \tau_{y}\right)}\right\} \cdot \sin \left[\beta_{h} \cdot\left(x-x_{l}\right)\right],
$$

the $y$-component of $\vec{B}$, according to (B3) with $B_{y}^{y}=0$, by

$$
B_{y}=-\sum_{h=1}^{\infty}\left\{c_{h}^{x} \cdot \frac{\operatorname{sh}\left[\beta_{h} \cdot\left(y_{t}-y\right)\right]}{\operatorname{sh}\left(\beta_{h} \cdot \tau_{y}\right)}+d_{h}^{x} \cdot \frac{\operatorname{sh}\left[\beta_{h} \cdot\left(y-y_{l}\right)\right]}{\operatorname{sh}\left(\beta_{h} \cdot \tau_{y}\right)}\right\} \cdot \cos \left[\beta_{h} \cdot\left(x-x_{l}\right)\right] .
$$

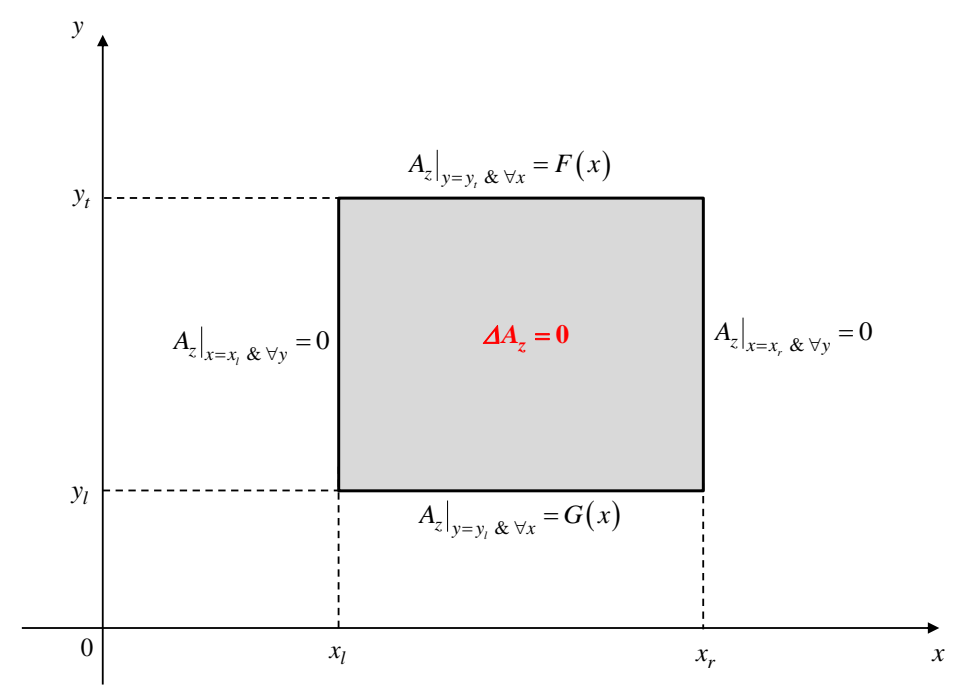

Figure B2. Particular case: $A_{z}=0$ on $x$-edges and $A_{z}$ imposed on $y$-edges of a region.

Appendix B.2. Case-Study No 2: $B_{y}$ and $A_{z}$ Are Respectively Imposed on X-and $Y$-Edges of a Region

Figure B3a shows a region for $\left(x \in\left[x_{l}, x_{r}\right]\right.$ and $\left.y \in\left[y_{l}, y_{t}\right]\right)$ whose the magnetic flux densities and vector potentials are respectively imposed on $x$ - and $y$-edges. By applying the principle of superposition on the magnetic quantities, Figure B3a is redefined by Figure B3b. 


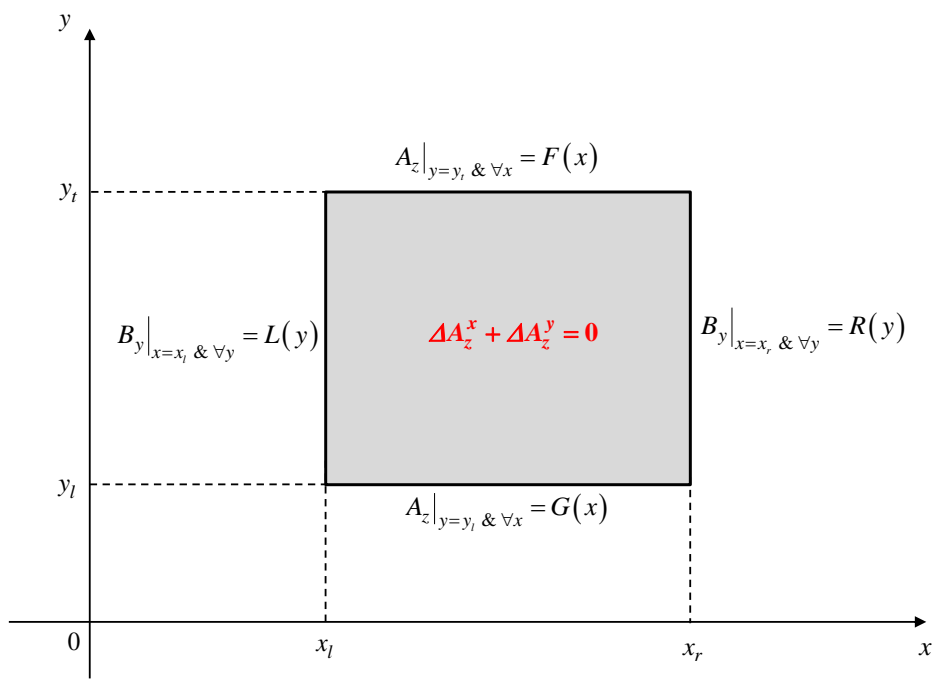

(a)

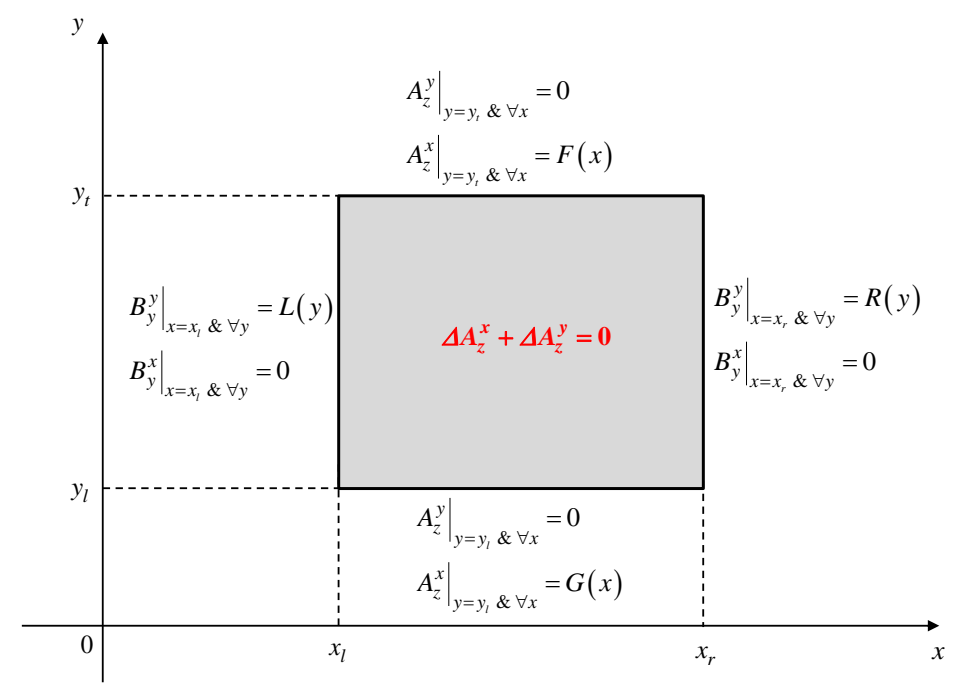

(b)

Figure B3. $B_{y}$ imposed on $x$-edges and $A_{z}$ imposed on $y$-edges of a region: (a) General and (b) Principle of superposition.

In the case-study no 2 , the magnetic vector potential $A_{z}=A_{z}^{x}+A_{z}^{y}$, i.e., (A5), is redefined by

$$
\begin{array}{r}
A_{z}^{x}=\mid \begin{array}{l}
\left(y_{t}-y\right) \cdot c_{0}^{x}+\left(y-y_{l}\right) \cdot d_{0}^{x} \\
\cdots+\sum_{h=1}^{\infty}\left\{\frac{c_{h}^{x}}{\beta_{h}} \cdot \frac{s h\left[\beta_{h} \cdot\left(y_{t}-y\right)\right]}{\operatorname{sh}\left(\beta_{h} \cdot \tau_{y}\right)}+\frac{d_{h}^{x}}{\beta_{h}} \cdot \frac{\operatorname{sh}\left[\beta_{h} \cdot\left(y-y_{l}\right)\right]}{\operatorname{sh}\left(\beta_{h} \cdot \tau_{y}\right)}\right\} \cdot \cos \left[\beta_{h} \cdot\left(x-x_{l}\right)\right],
\end{array} \\
A_{z}^{y}=-\sum_{n=1}^{\infty}\left\{\frac{e_{n}^{y}}{\lambda_{n}} \cdot \frac{\operatorname{ch}\left[\lambda_{n} \cdot\left(x-x_{l}\right)\right]}{\operatorname{sh}\left(\lambda_{n} \cdot \tau_{x}\right)}-\frac{f_{n}^{y}}{\lambda_{n}} \cdot \frac{\operatorname{ch}\left[\lambda_{n} \cdot\left(x_{r}-x\right)\right]}{\operatorname{sh}\left(\lambda_{n} \cdot \tau_{x}\right)}\right\} \cdot \sin \left[\lambda_{n} \cdot\left(y-y_{l}\right)\right],
\end{array}
$$

the component $B_{x}=B_{x}^{x}+B_{x}^{y}$ of $\vec{B}$, i.e., (A7), by

$$
B_{x}^{x}=\mid \begin{aligned}
& -c_{0}^{x}+d_{0}^{x} \\
& \cdots+\sum_{h=1}^{\infty}\left\{-c_{h}^{x} \cdot \frac{\operatorname{ch}\left[\beta_{h} \cdot\left(y_{t}-y\right)\right]}{\operatorname{sh}\left(\beta_{h} \cdot \tau_{y}\right)}+d_{h}^{x} \cdot \frac{\operatorname{ch}\left[\beta_{h} \cdot\left(y-y_{l}\right)\right]}{\operatorname{sh}\left(\beta_{h} \cdot \tau_{y}\right)}\right\} \cdot \cos \left[\beta_{h} \cdot\left(x-x_{l}\right)\right],
\end{aligned},
$$




$$
B_{x}^{y}=-\sum_{n=1}^{\infty}\left\{e_{n}^{y} \cdot \frac{\operatorname{ch}\left[\lambda_{n} \cdot\left(x-x_{l}\right)\right]}{\operatorname{sh}\left(\lambda_{n} \cdot \tau_{x}\right)}-f_{n}^{y} \cdot \frac{\operatorname{ch}\left[\lambda_{n} \cdot\left(x_{r}-x\right)\right]}{\operatorname{sh}\left(\lambda_{n} \cdot \tau_{x}\right)}\right\} \cdot \cos \left[\lambda_{n} \cdot\left(y-y_{l}\right)\right],
$$

and the component $B_{y}=B_{y}^{x}+B_{y}^{y}$ of $\vec{B}$, i.e., (A8), by

$$
\begin{aligned}
& B_{y}^{x}=\sum_{h=1}^{\infty}\left\{c_{h}^{x} \cdot \frac{\operatorname{sh}\left[\beta_{h} \cdot\left(y_{t}-y\right)\right]}{\operatorname{sh}\left(\beta_{h} \cdot \tau_{y}\right)}+d_{h}^{x} \cdot \frac{\operatorname{sh}\left[\beta_{h} \cdot\left(y-y_{l}\right)\right]}{\operatorname{sh}\left(\beta_{h} \cdot \tau_{y}\right)}\right\} \cdot \sin \left[\beta_{h} \cdot\left(x-x_{l}\right)\right], \\
& B_{y}^{y}=\sum_{n=1}^{\infty}\left\{e_{n}^{y} \cdot \frac{\operatorname{sh}\left[\lambda_{n} \cdot\left(x-x_{l}\right)\right]}{\operatorname{sh}\left(\lambda_{n} \cdot \tau_{x}\right)}+f_{n}^{y} \cdot \frac{\operatorname{sh}\left[\lambda_{n} \cdot\left(x_{r}-x\right)\right]}{\operatorname{sh}\left(\lambda_{n} \cdot \tau_{x}\right)}\right\} \cdot \sin \left[\lambda_{n} \cdot\left(y-y_{l}\right)\right],
\end{aligned}
$$

where $c_{0}^{x}, d_{0}^{x}, c_{h}^{x}, d_{h}^{x}, e_{n}^{y}$ and $f_{n}^{y}$ are new integration constants.

\section{Appendix C. Elements of Linear Systems}

Appendix C.1. Simplifying Function of General Integrals

For the determination of the integral constants, it is required to calculate general integrals of the form

$$
\begin{aligned}
& F_{s}=\int_{l_{l}}^{l_{l}+w} \sin \left[\alpha_{s} \cdot\left(l-l_{s}\right)\right] \cdot d l, \\
& F_{c s}=\int_{l_{l}}^{l_{l}+w} \cos \left[\alpha_{c} \cdot\left(l-l_{c}\right)\right] \cdot \sin \left[\alpha_{s} \cdot\left(l-l_{s}\right)\right] \cdot d l, \\
& F_{s s}=\int_{l_{l}}^{l_{l}+w} \sin \left[\alpha_{s 1} \cdot\left(l-l_{s 1}\right)\right] \cdot \sin \left[\alpha_{s 2} \cdot\left(l-l_{s 2}\right)\right] \cdot d l, \\
& F_{l s}=\int_{l_{l}}^{l_{l}+w} l \cdot \sin \left[\alpha_{s} \cdot\left(l-l_{s}\right)\right] \cdot d l, \\
& F_{l 2 s}=\int_{l_{l}}^{l_{l}+w} l^{2} \cdot \sin \left[\alpha_{s} \cdot\left(l-l_{s}\right)\right] \cdot d l, \\
& F_{c h s}=\int_{l_{l}}^{l_{l}+w} \operatorname{ch}\left[\alpha_{c h} \cdot\left(l-l_{c h}\right)\right] \cdot \sin \left[\alpha_{s} \cdot\left(l-l_{s}\right)\right] \cdot d l \text {, } \\
& F_{s h s}=\int_{l_{l}}^{l_{l}+w} \operatorname{sh}\left[\alpha_{s h} \cdot\left(l-l_{s h}\right)\right] \cdot \sin \left[\alpha_{s} \cdot\left(l-l_{s}\right)\right] \cdot d l .
\end{aligned}
$$

The functions (C1) will be used in the expression of the integration constants. The expressions of (C1a)-(C1c) have given in [68]. The development of (C1d)-(C1g) gives

$$
F_{l s}\left(\alpha_{s}, l_{s}, l_{l}, w\right)=\frac{\left[\begin{array}{l}
\sin \left[\alpha_{s} \cdot\left(l_{l}+w-l_{s}\right)\right]-\sin \left[\alpha_{s} \cdot\left(l_{l}-l_{s}\right)\right] \\
\cdots-\alpha_{s} \cdot\left\{\left(l_{l}+w\right) \cdot \cos \left[\alpha_{s} \cdot\left(l_{l}+w-l_{s}\right)\right]-l_{l} \cdot \cos \left[\alpha_{s} \cdot\left(l_{l}-l_{s}\right)\right]\right\}
\end{array}\right]}{\alpha_{s}^{2}},
$$




$$
\begin{aligned}
& F_{l 2 s}\left(\alpha_{s}, l_{s}, l_{l}, w\right)=\frac{\left[\begin{array}{l}
2 \cdot\left\{\cos \left[\alpha_{s} \cdot\left(l_{l}+w-l_{s}\right)\right]-\cos \left[\alpha_{s} \cdot\left(l_{l}-l_{s}\right)\right]\right\} \\
\cdots-\alpha_{s}^{2} \cdot\left\{\left(l_{l}+w\right)^{2} \cdot \cos \left[\alpha_{s} \cdot\left(l_{l}+w-l_{s}\right)\right]-l_{l}^{2} \cdot \cos \left[\alpha_{s} \cdot\left(l_{l}-l_{s}\right)\right]\right\} \\
\cdots+2 \cdot \alpha_{s} \cdot\left\{\left(l_{l}+w\right) \cdot \sin \left[\alpha_{s} \cdot\left(l_{l}+w-l_{s}\right)\right]-l_{l} \cdot \sin \left[\alpha_{s} \cdot\left(l_{l}-l_{s}\right)\right]\right\}
\end{array}\right]}{\alpha_{s}^{3}}, \\
& F_{c h s}\left(\alpha_{c h}, \alpha_{s}, l_{c h}, l_{s}, l_{l}, w\right)=\frac{\left[\begin{array}{l}
-\alpha_{c h} \cdot\left\{\begin{array}{l}
\operatorname{sh}\left[\alpha_{c h} \cdot\left(l_{l}-l_{c h}\right)\right] \cdot \sin \left[\alpha_{s} \cdot\left(l_{l}-l_{s}\right)\right] \\
\cdots-\operatorname{sh}\left[\alpha_{c h} \cdot\left(l_{l}+w-l_{c h}\right)\right] \cdot \sin \left[\alpha_{s} \cdot\left(l_{l}+w-l_{s}\right)\right]
\end{array}\right\} \\
\cdots+\alpha_{s} \cdot\left\{\begin{array}{l}
c h\left[\alpha_{c h} \cdot\left(l_{l}-l_{c h}\right)\right] \cdot \cos \left[\alpha_{s} \cdot\left(l_{l}-l_{s}\right)\right] \\
\cdots-c h\left[\alpha_{c h} \cdot\left(l_{l}+w-l_{c h}\right)\right] \cdot \cos \left[\alpha_{s} \cdot\left(l_{l}+w-l_{s}\right)\right]
\end{array}\right\}
\end{array}\right]}{\left(\alpha_{c h}^{2}+\alpha_{s}^{2}\right)} \\
& F_{s h s}\left(\alpha_{s h}, \alpha_{s}, l_{s h}, l_{s}, l_{l}, w\right)=\frac{\left[\begin{array}{l}
-\alpha_{s h} \cdot\left\{\begin{array}{l}
\operatorname{ch}\left[\alpha_{s h} \cdot\left(l_{l}-l_{s h}\right)\right] \cdot \sin \left[\alpha_{s} \cdot\left(l_{l}-l_{s}\right)\right] \\
\cdots-\operatorname{ch}\left[\alpha_{s h} \cdot\left(l_{l}+w-l_{s h}\right)\right] \cdot \sin \left[\alpha_{s} \cdot\left(l_{l}+w-l_{s}\right)\right]
\end{array}\right\} \\
\cdots+\alpha_{s} \cdot\left\{\begin{array}{l}
\operatorname{sh}\left[\alpha_{s h} \cdot\left(l_{l}-l_{s h}\right)\right] \cdot \cos \left[\alpha_{s} \cdot\left(l_{l}-l_{s}\right)\right] \\
\cdots-\operatorname{sh}\left[\alpha_{s h} \cdot\left(l_{l}+w-l_{s h}\right)\right] \cdot \cos \left[\alpha_{s} \cdot\left(l_{l}+w-l_{s}\right)\right]
\end{array}\right\}
\end{array}\right]}{\left(\alpha_{s h}^{2}+\alpha_{s}^{2}\right)} .
\end{aligned}
$$

Appendix C.2. Expression of $d 1_{h 1}^{x}$ for Region 1

By incorporating $F_{1}(x)$ (see Figure 4a) into (7) and by using (13), (18), (23), (28) and (33), the development of (7) gives

$$
\begin{aligned}
& \mid \sum_{h 3=1}^{\infty}\left(c 3_{h 3}^{x} \cdot Q 13 c_{h 1, h 3}+d 3_{h 3}^{x} \cdot Q 13 d_{h 1, h 3}\right)+\sum_{n 3=1}^{\infty} f 3_{n 3}^{y} \cdot Q 13 f_{h 1, n 3} \\
& \cdots+\sum_{h 4=1}^{\infty}\left(c 4_{h 4}^{x} \cdot Q 14_{h 1, h 4}+d 4_{h 4}^{x} \cdot Q 14 d_{h 1, h 4}\right)+\sum_{n 4=1}^{\infty} e 4_{n 4}^{y} \cdot Q 14 e_{h 1, n 4} \\
& d 1_{h 1}^{x}=-\begin{array}{l}
\cdots+\sum_{h 5=1}^{\infty}\left(c 5_{h 5}^{x} \cdot Q 15 c_{h 1, h 5}+d 5_{h 5}^{x} \cdot Q 15 d_{h 1, h 5}\right)+\sum_{n=1}^{\infty}\left(e 5_{n 5}^{y} \cdot Q 15 e_{h 1, n 5}+f 5_{n 5}^{y} \cdot Q 15 f_{h 1, n 5}\right) \\
\cdots+\sum_{h 6=0}^{\infty}\left(c 6_{h 6}^{x} \cdot Q 16 c_{h 1, h 6}+d 6_{h 6}^{x} \cdot Q 16 d_{h 1, h 6}\right)+\sum_{n 6=1}^{\infty}\left(e 6_{n 6}^{y} \cdot Q 16 e_{h 1, n 6}+f 6_{n 6}^{y} \cdot Q 16 f_{h 1, n 6}\right)
\end{array}, \\
& \begin{array}{l}
\cdots+\sum_{h 7=0}^{\infty}\left(c 7_{h 7}^{x} \cdot Q 17 c_{h 1, h 7}+d 7_{h 7}^{x} \cdot Q 17 d_{h 1, h 7}\right)+\sum_{n 7=1}^{\infty}\left(e 7_{n 7}^{y} \cdot Q 17 e_{h 1, n 7}+f 6_{n 7}^{y} \cdot Q 17 f_{h 1, n 7}\right) \\
\cdots-E S 1_{h 1}
\end{array} \\
& Q 13 c_{h 1, h 3}=\frac{2}{\tau_{x 1}} \cdot \frac{\mu_{1}}{\mu_{3}} \cdot \operatorname{coth}\left(\beta 3_{h 3} \cdot \tau_{y 3}\right) \cdot F_{s s}\left(\beta 3_{h 3}, \beta 1_{h 1}, x_{1}, x_{1}, x_{1}, \tau_{x 3}\right), \\
& Q 13 d_{h 1, h 3}=-\frac{2}{\tau_{x 1}} \cdot \frac{\mu_{1}}{\mu_{3}} \cdot \operatorname{csch}\left(\beta 3_{h 3} \cdot \tau_{y 3}\right) \cdot F_{s s}\left(\beta 3_{h 3}, \beta 1_{h 1}, x_{1}, x_{1}, x_{1}, \tau_{x 3}\right), \\
& Q 13 f_{h 1, n 3}=-\frac{2}{\tau_{x 1}} \cdot \frac{\mu_{1}}{\mu_{3}} \cdot \operatorname{csch}\left(\lambda 3_{n 3} \cdot \tau_{x 3}\right) \cdot F_{s h s}\left(\lambda 3_{n 3}, \beta 1_{h 1}, x_{1}, x_{1}, x_{1}, \tau_{x 3}\right) \text {, } \\
& Q 14 c_{h 1, h 4}=\frac{2}{\tau_{x 1}} \cdot \frac{\mu_{1}}{\mu_{4}} \cdot \operatorname{coth}\left(\beta 4_{h 4} \cdot \tau_{y 4}\right) \cdot F_{s s}\left(\beta 4_{h 4}, \beta 1_{h 1}, x_{5}, x_{1}, x_{5}, \tau_{x 4}\right), \\
& Q 14 d_{h 1, h 4}=-\frac{2}{\tau_{x 1}} \cdot \frac{\mu_{1}}{\mu_{4}} \cdot \operatorname{csch}\left(\beta 4_{h 4} \cdot \tau_{y 4}\right) \cdot F_{s s}\left(\beta 4_{h 4}, \beta 1_{h 1}, x_{5}, x_{1}, x_{5}, \tau_{x 4}\right), \\
& Q 14 e_{h 1, n 4}=\frac{2}{\tau_{x 1}} \cdot \frac{\mu_{1}}{\mu_{4}} \cdot \operatorname{csch}\left(\lambda 4_{n 4} \cdot \tau_{x 4}\right) \cdot F_{s h s}\left(\lambda 4_{n 4}, \beta 1_{h 1}, x_{6}, x_{1}, x_{5}, \tau_{x 4}\right) \text {, } \\
& Q 15 c_{h 1, h 5}=\frac{2}{\tau_{x 1}} \cdot \frac{\mu_{1}}{\mu_{5}} \cdot \operatorname{coth}\left(\beta 5_{h 5} \cdot \tau_{y 5}\right) \cdot F_{s s}\left(\beta 5_{h 5}, \beta 1_{h 1}, x_{3}, x_{1}, x_{3}, \tau_{x 5}\right) \text {, } \\
& Q 15 d_{h 1, h 5}=-\frac{2}{\tau_{x 1}} \cdot \frac{\mu_{1}}{\mu_{5}} \cdot \operatorname{csch}\left(\beta 5_{h 5} \cdot \tau_{y 5}\right) \cdot F_{s s}\left(\beta 5_{h 5}, \beta 1_{h 1}, x_{3}, x_{1}, x_{3}, \tau_{x 5}\right) \text {, } \\
& Q 15 e_{h 1, n 5}=\frac{2}{\tau_{x 1}} \cdot \frac{\mu_{1}}{\mu_{5}} \cdot \operatorname{csch}\left(\lambda 5_{n 5} \cdot \tau_{x 5}\right) \cdot F_{s h s}\left(\lambda 5_{n 5}, \beta 1_{h 1}, x_{4}, x_{1}, x_{3}, \tau_{x 5}\right) \text {, }
\end{aligned}
$$




$$
\begin{aligned}
& Q 15 f_{h 1, n 5}=-\frac{2}{\tau_{x 1}} \cdot \frac{\mu_{1}}{\mu_{5}} \cdot \operatorname{csch}\left(\lambda 5_{n 5} \cdot \tau_{x 5}\right) \cdot F_{s h s}\left(\lambda 5_{n 5}, \beta 1_{h 1}, x_{3}, x_{1}, x_{3}, \tau_{x 5}\right) \text {, } \\
& Q 16 c_{h 1, h 6}=\frac{2}{\tau_{x 1}} \cdot \frac{\mu_{1}}{\mu_{6}} \cdot \begin{cases}F_{s}\left(\beta 1_{h 1}, x_{1}, x_{2}, \tau_{x 6}\right) & \text { for } h 6=0 \\
\operatorname{coth}\left(\beta 6_{h 6} \cdot \tau_{y 6}\right) \cdot F_{c s}\left(\beta 6_{h 6}, \beta 1_{h 1}, x_{2}, x_{1}, x_{2}, \tau_{x 6}\right) & \text { for } h 6 \neq 0\end{cases} \\
& Q 16 d_{h 1, h 6}=-\frac{2}{\tau_{x 1}} \cdot \frac{\mu_{1}}{\mu_{6}} \cdot \begin{cases}F_{s}\left(\beta 1_{h 1}, x_{1}, x_{2}, \tau_{x 6}\right) & \text { for } h 6=0 \\
\operatorname{csch}\left(\beta 6_{h 6} \cdot \tau_{y 6}\right) \cdot F_{c s}\left(\beta 6_{h 6}, \beta 1_{h 1}, x_{2}, x_{1}, x_{2}, \tau_{x 6}\right) & \text { for } h 6 \neq 0\end{cases} \\
& Q 16 e_{h 1, n 6}=\frac{2}{\tau_{x 1}} \cdot \frac{\mu_{1}}{\mu_{6}} \cdot \operatorname{csch}\left(\lambda 6_{n 6} \cdot \tau_{x 6}\right) \cdot F_{c h s}\left(\lambda 6_{n 6}, \beta 1_{h 1}, x_{2}, x_{1}, x_{2}, \tau_{x 6}\right) \text {, } \\
& Q 16 f_{h 1, n 6}=-\frac{2}{\tau_{x 1}} \cdot \frac{\mu_{1}}{\mu_{6}} \cdot \operatorname{csch}\left(\lambda 6_{n 6} \cdot \tau_{x 6}\right) \cdot F_{c h s}\left(\lambda 6_{n 6}, \beta 1_{h 1}, x_{3}, x_{1}, x_{2}, \tau_{x 6}\right) \text {, } \\
& Q 1 c_{h 1, h 7}=\frac{2}{\tau_{x 1}} \cdot \frac{\mu_{1}}{\mu_{7}} \cdot \begin{cases}F_{S}\left(\beta 1_{h 1}, x_{1}, x_{4}, \tau_{x 7}\right) & \text { for } h 7=0 \\
\operatorname{coth}\left(\beta 7_{h 7} \cdot \tau_{y 7}\right) \cdot F_{c s}\left(\beta 7_{h 7}, \beta 1_{h 1}, x_{4}, x_{1}, x_{4}, \tau_{x 7}\right) & \text { for } h 7 \neq 0\end{cases} \\
& Q 17 d_{h 1, h 7}=-\frac{2}{\tau_{x 1}} \cdot \frac{\mu_{1}}{\mu_{7}} \cdot \begin{cases}F_{S}\left(\beta 1_{h 1}, x_{1}, x_{4}, \tau_{x 7}\right) & \text { for } h 7=0 \\
\operatorname{csch}\left(\beta 7_{h 7} \cdot \tau_{y 7}\right) \cdot F_{c s}\left(\beta 7_{h 7}, \beta 1_{h 1}, x_{4}, x_{1}, x_{4}, \tau_{x 7}\right) & \text { for } h 7 \neq 0\end{cases} \\
& Q 17 e_{h 1, n 7}=\frac{2}{\tau_{x 1}} \cdot \frac{\mu_{1}}{\mu_{7}} \cdot \operatorname{csch}\left(\lambda 7_{n 7} \cdot \tau_{x 7}\right) \cdot F_{c h s}\left(\lambda 7_{n 7}, \beta 1_{h 1}, x_{4}, x_{1}, x_{4}, \tau_{x 7}\right) \text {, } \\
& Q 17 f_{h 1, n 7}=-\frac{2}{\tau_{x 1}} \cdot \frac{\mu_{1}}{\mu_{7}} \cdot \operatorname{csch}\left(\lambda 7_{n 7} \cdot \tau_{x 7}\right) \cdot F_{c h s}\left(\lambda 7_{n 7}, \beta 1_{h 1}, x_{5}, x_{1}, x_{4}, \tau_{x 7}\right) \text {, } \\
& E S 16_{h 1}=\left.\frac{2}{\tau_{x 1}} \cdot \frac{\mu_{1}}{\mu_{6}} \cdot B_{x P 6}\right|_{y=y_{2}} \cdot F_{S}\left(\beta 1_{h 1}, x_{1}, x_{2}, \tau_{x 6}\right), \\
& E S 17_{h 1}=\left.\frac{2}{\tau_{x 1}} \cdot \frac{\mu_{1}}{\mu_{7}} \cdot B_{x P 7}\right|_{y=y_{2}} \cdot F_{S}\left(\beta 1_{h 1}, x_{1}, x_{4}, \tau_{x 7}\right) .
\end{aligned}
$$

Appendix C.3. Expression of $c 2_{h 2}^{x}$ for Region 2

By incorporating $G_{2}(x)$ (see Figure 4b) into (11) and by using (13), (18), (23), (28) and (33), the development of (11) gives

$$
\begin{gathered}
c 2_{h 2}^{x}=-\mid \begin{array}{l}
\sum_{h 3=1}^{\infty}\left(c 3_{h 3}^{x} \cdot Q 23 c_{h 2, h 3}+d 3_{h 3}^{x} \cdot Q 23 d_{h 2, h 3}\right)+\sum_{n 3=1}^{\infty} f 3_{n 3}^{y} \cdot Q 23 f_{h 2, n 3} \\
\cdots+\sum_{h 4=1}^{\infty}\left(c 4_{h 4}^{x} \cdot Q 24 c_{h 2, h 4}+d 4_{h 4}^{x} \cdot Q 24 d_{h 2, h 4}\right)+\sum_{n=1}^{\infty} e 4_{n 4}^{y} \cdot Q 24 e_{h 2, n 4} \\
\cdots+\sum_{h 5=1}^{\infty}\left(c 5_{h 5}^{x} \cdot Q 25 c_{h 2, h 5}+d 5_{h 5}^{x} \cdot Q 25 d_{h 2, h 5}\right)+\sum_{n=1}^{\infty}\left(e 5_{n 5}^{y} \cdot Q 25 e_{h 2, n 5}+f 5_{n 5}^{y} \cdot Q 25 f_{h 2, n 5}\right) \\
\cdots+\sum_{h 6=0}^{\infty}\left(c 6_{h 6}^{x} \cdot Q 26 c_{h 2, h 6}+d 6_{h 6}^{x} \cdot Q 26 d_{h 2, h 6}\right)+\sum_{n 6=1}^{\infty}\left(e 6_{n 6}^{y} \cdot Q 26 e_{h 2, n 6}+f 6_{n 6}^{y} \cdot Q 26 f_{h 2, n 6}\right) \\
\cdots+\sum_{h 7=0}^{\infty}\left(c 7_{h 7}^{x} \cdot Q 27 c_{h 2, h 7}+d 7_{h 7}^{x} \cdot Q 27 d_{h 2, h 7}\right)+\sum_{n 7=1}^{\infty}\left(e 7_{n 7}^{y} \cdot Q 27 e_{h 2, n 7}+f 6_{n 7}^{y} \cdot Q 27 f_{h 2, n 7}\right) \\
\cdots-\left(E S 26_{h 2}+E S 27_{h 2}\right)
\end{array} \\
Q 23 c_{h 2, h 3}=\frac{2}{\tau_{x 2}} \cdot \frac{\mu_{2}}{\mu_{3}} \cdot \operatorname{csch}\left(\beta 3_{h 3} \cdot \tau_{y 3}\right) \cdot F_{s s}\left(\beta 3_{h 3}, \beta 2_{h 2}, x_{1}, x_{1}, x_{1}, \tau_{x 3}\right), \\
Q 23 d_{h 2, h 3}=-\frac{2}{\tau_{x 2}} \cdot \frac{\mu_{2}}{\mu_{3}} \cdot \operatorname{coth}\left(\beta 3_{h 3} \cdot \tau_{y 3}\right) \cdot F_{s s}\left(\beta 3_{h 3}, \beta 2_{h 2}, x_{1}, x_{1}, x_{1}, \tau_{x 3}\right), \\
Q 23 f_{h 2, n 3}=-\frac{2}{\tau_{x 2}} \cdot \frac{\mu_{2}}{\mu_{3}} \cdot \frac{\cos \left(\lambda 3_{n 3} \cdot \tau_{y 3}\right)}{\operatorname{sh}\left(\lambda_{n 3} \cdot \tau_{x 3}\right)} \cdot F_{s h s}\left(\lambda 3_{n 3}, \beta 2_{h 2}, x_{1}, x_{1}, x_{1}, \tau_{x 3}\right), \\
Q 24 c_{h 2, h 4}=\frac{2}{\tau_{x 2}} \cdot \frac{\mu_{2}}{\mu_{4}} \cdot \operatorname{csch}\left(\beta 4_{h 4} \cdot \tau_{y 4}\right) \cdot F_{s s}\left(\beta 4_{h 4}, \beta 2_{h 2}, x_{5}, x_{1}, x_{5}, \tau_{x 4}\right),
\end{gathered}
$$




$$
\begin{aligned}
& Q 24 d_{h 2, h 4}=-\frac{2}{\tau_{x 2}} \cdot \frac{\mu_{2}}{\mu_{4}} \cdot \operatorname{coth}\left(\beta 4_{h 4} \cdot \tau_{y 4}\right) \cdot F_{S S}\left(\beta 4_{h 4}, \beta 2_{h 2}, x_{5}, x_{1}, x_{5}, \tau_{x 4}\right), \\
& Q 24 e_{h 2, n 4}=\frac{2}{\tau_{x 2}} \cdot \frac{\mu_{2}}{\mu_{4}} \cdot \frac{\cos \left(\lambda 4_{n 4} \cdot \tau_{y 4}\right)}{\operatorname{sh}\left(\lambda 4_{n 4} \cdot \tau_{x 4}\right)} \cdot F_{s h s}\left(\lambda 4_{n 4}, \beta 2_{h 2}, x_{6}, x_{1}, x_{5}, \tau_{x 4}\right) \text {, } \\
& Q 25 c_{h 2, h 5}=\frac{2}{\tau_{x 2}} \cdot \frac{\mu_{2}}{\mu_{5}} \cdot \operatorname{csch}\left(\beta 5_{h 5} \cdot \tau_{y 5}\right) \cdot F_{s s}\left(\beta 5_{h 5}, \beta 2_{h 2}, x_{3}, x_{1}, x_{3}, \tau_{x 5}\right) \text {, } \\
& Q 25 d_{h 2, h 5}=-\frac{2}{\tau_{x 2}} \cdot \frac{\mu_{2}}{\mu_{5}} \cdot \operatorname{coth}\left(\beta 5_{h 5} \cdot \tau_{y 5}\right) \cdot F_{s S}\left(\beta 5_{h 5}, \beta 2_{h 2}, x_{3}, x_{1}, x_{3}, \tau_{x 5}\right) \text {, } \\
& Q 25 e_{h 2, n 5}=\frac{2}{\tau_{x 2}} \cdot \frac{\mu_{2}}{\mu_{5}} \cdot \frac{\cos \left(\lambda 5_{n 5} \cdot \tau_{y 5}\right)}{\operatorname{sh}\left(\lambda 5_{n 5} \cdot \tau_{x 5}\right)} \cdot F_{s h s}\left(\lambda 5_{n 5}, \beta 2_{h 2}, x_{4}, x_{1}, x_{3}, \tau_{x 5}\right) \text {, } \\
& Q 25 f_{h 2, n 5}=-\frac{2}{\tau_{x 2}} \cdot \frac{\mu_{2}}{\mu_{5}} \cdot \frac{\cos \left(\lambda 5_{n 5} \cdot \tau_{y 5}\right)}{\operatorname{sh}\left(\lambda 5_{n 5} \cdot \tau_{x 5}\right)} \cdot F_{s h s}\left(\lambda 5_{n 5}, \beta 2_{h 2}, x_{3}, x_{1}, x_{3}, \tau_{x 5}\right) \text {, } \\
& Q 26 c_{h 2, h 6}=\frac{2}{\tau_{x 2}} \cdot \frac{\mu_{2}}{\mu_{6}} \cdot \begin{cases}F_{s}\left(\beta 2_{h 2}, x_{1}, x_{2}, \tau_{x 6}\right) & \text { for } h 6=0 \\
\operatorname{csch}\left(\beta 6_{h 6} \cdot \tau_{y 6}\right) \cdot F_{c s}\left(\beta 6_{h 6}, \beta 2_{h 2}, x_{2}, x_{1}, x_{2}, \tau_{x 6}\right) & \text { for } h 6 \neq 0\end{cases} \\
& Q 26 d_{h 2, h 6}=-\frac{2}{\tau_{x 2}} \cdot \frac{\mu_{2}}{\mu_{6}} \cdot \begin{cases}F_{s}\left(\beta 2_{h 2}, x_{1}, x_{2}, \tau_{x 6}\right) & \text { for } h 6=0 \\
\operatorname{coth}\left(\beta 6_{h 6} \cdot \tau_{y 6}\right) \cdot F_{c s}\left(\beta 6_{h 6}, \beta 2_{h 2}, x_{2}, x_{1}, x_{2}, \tau_{x 6}\right) & \text { for } h 6 \neq 0\end{cases} \\
& Q 26 e_{h 2, n 6}=\frac{2}{\tau_{x 2}} \cdot \frac{\mu_{2}}{\mu_{6}} \cdot \frac{\cos \left(\lambda 6_{n 6} \cdot \tau_{y 6}\right)}{\operatorname{sh}\left(\lambda 6_{n 6} \cdot \tau_{x 6}\right)} \cdot F_{c h s}\left(\lambda 6_{n 6}, \beta 2_{h 2}, x_{2}, x_{1}, x_{2}, \tau_{x 6}\right) \text {, } \\
& Q 26 f_{h 2, n 6}=-\frac{2}{\tau_{x 2}} \cdot \frac{\mu_{2}}{\mu_{6}} \cdot \frac{\cos \left(\lambda 6_{n 6} \cdot \tau_{y 6}\right)}{\operatorname{sh}\left(\lambda 6_{n 6} \cdot \tau_{x 6}\right)} \cdot F_{c h s}\left(\lambda 6_{n 6}, \beta 2_{h 2}, x_{3}, x_{1}, x_{2}, \tau_{x 6}\right) \text {, } \\
& Q 27 c_{h 2, h 7}=\frac{2}{\tau_{x 2}} \cdot \frac{\mu_{2}}{\mu_{7}} \cdot \begin{cases}F_{s}\left(\beta 2_{h 2}, x_{1}, x_{4}, \tau_{x 7}\right) & \text { for } h 7=0 \\
\operatorname{csch}\left(\beta 7_{h 7} \cdot \tau_{y 7}\right) \cdot F_{c s}\left(\beta 7_{h 7}, \beta 2_{h 2}, x_{4}, x_{1}, x_{4}, \tau_{x 7}\right) & \text { for } h 7 \neq 0\end{cases} \\
& Q 27 d_{h 2, h 7}=-\frac{2}{\tau_{x 2}} \cdot \frac{\mu_{2}}{\mu_{7}} \cdot \begin{cases}F_{s}\left(\beta 2_{h 2}, x_{1}, x_{4}, \tau_{x 7}\right) & \text { for } h 7=0 \\
\operatorname{coth}\left(\beta 7_{h 7} \cdot \tau_{y 7}\right) \cdot F_{c S}\left(\beta 7_{h 7}, \beta 2_{h 2}, x_{4}, x_{1}, x_{4}, \tau_{x 7}\right) & \text { for } h 7 \neq 0\end{cases} \\
& Q 27 e_{h 2, n 7}=\frac{2}{\tau_{x 2}} \cdot \frac{\mu_{2}}{\mu_{7}} \cdot \frac{\cos \left(\lambda 7_{n 7} \cdot \tau_{y 7}\right)}{\operatorname{sh}\left(\lambda 7_{n 7} \cdot \tau_{x 7}\right)} \cdot F_{c h s}\left(\lambda 7_{n 7}, \beta 2_{h 2}, x_{4}, x_{1}, x_{4}, \tau_{x 7}\right), \\
& Q 27 f_{h 2, n 7}=-\frac{2}{\tau_{x 2}} \cdot \frac{\mu_{2}}{\mu_{7}} \cdot \frac{\cos \left(\lambda 7_{n 7} \cdot \tau_{y 7}\right)}{\operatorname{sh}\left(\lambda 7_{n 7} \cdot \tau_{x 7}\right)} \cdot F_{c h s}\left(\lambda 7_{n 7}, \beta 2_{h 2}, x_{5}, x_{1}, x_{4}, \tau_{x 7}\right) \text {, } \\
& E S 26_{h 2}=\left.\frac{2}{\tau_{x 2}} \cdot \frac{\mu_{2}}{\mu_{6}} \cdot B_{x P 6}\right|_{y=y_{3}} \cdot F_{S}\left(\beta 2_{h 2}, x_{1}, x_{2}, \tau_{x 6}\right) \text {, } \\
& E S 27_{h 2}=\left.\frac{2}{\tau_{x 2}} \cdot \frac{\mu_{2}}{\mu_{7}} \cdot B_{x P 7}\right|_{y=y_{3}} \cdot F_{S}\left(\beta 2_{h 2}, x_{1}, x_{4}, \tau_{x 7}\right) .
\end{aligned}
$$

Appendix C.4. Expression of $c 3_{h 3}^{x}, d 3_{h 3}^{x}$ and $f 3_{n 3}^{y}$ for Region 3

By using (4), the development of (15a) gives

$$
\begin{gathered}
c 3_{h 3}^{x}=-\sum_{h 1=1}^{\infty} d 1_{h 1}^{x} \cdot Q 31 d_{h 3, h 1}, \\
Q 31 d_{h 3, h 1}=-\frac{2}{\tau_{x 3}} \cdot \frac{\beta 3_{h 3}}{\beta 1_{h 1}} \cdot \operatorname{th}\left(\beta 1_{h 1} \cdot \tau_{y 1}\right) \cdot F_{s s}\left(\beta 1_{h 1}, \beta 3_{h 3}, x_{1}, x_{1}, x_{1}, \tau_{x 3}\right) .
\end{gathered}
$$


By using (8), the development of (15b) gives

$$
\begin{gathered}
d 3_{h 3}^{x}=-\sum_{h 2=1}^{\infty} c 2_{h 2}^{x} \cdot Q 32 c_{h 3, h 2}, \\
Q 32 c_{h 3, h 2}=\frac{2}{\tau_{x 3}} \cdot \frac{\beta 3_{h 3}}{\beta 2_{h 2}} \cdot \operatorname{th}\left(\beta 2_{h 2} \cdot \tau_{y 2}\right) \cdot F_{s s}\left(\beta 2_{h 2}, \beta 3_{h 3}, x_{1}, x_{1}, x_{1}, \tau_{x 3}\right) .
\end{gathered}
$$

By using (27), the development of (16) gives

$$
\begin{aligned}
& f 3_{n 3}^{y}=E S 36_{n 3}-\left|\begin{array}{l}
\sum_{h 6=0}^{\infty}\left(c 6_{h 6}^{x} \cdot Q 36 c_{n 3, h 6}+d 6_{h 6}^{x} \cdot Q 36 d_{n 3, h 6}\right) \\
\cdots+\sum_{n 6=1}^{\infty}\left(e 6_{n 6}^{y} \cdot Q 36 e_{n 3, n 6}+f 6_{n 6}^{y} \cdot Q 36 f_{n 3, n 6}\right)
\end{array},\right| \\
& Q 36 c_{n 3, h 6}=\frac{2}{\tau_{y 3}} \cdot \lambda 3_{n 3} \cdot \begin{cases}-\left[y_{3} \cdot F_{s}\left(\lambda 3_{n 3}, y_{2}, y_{2}, \tau_{y 3}\right)-F_{l s}\left(\lambda 3_{n 3}, y_{2}, y_{2}, \tau_{y 3}\right)\right] & \text { for } h 6=0 \\
\frac{1}{\beta 6_{h 6}} \cdot \operatorname{csch}\left(\beta 6_{h 6} \cdot \tau_{y 6}\right) \cdot F_{s h s}\left(\beta 6_{h 6}, \lambda 3_{n 3}, y_{3}, y_{2}, y_{2}, \tau_{y 3}\right) & \text { for } h 6 \neq 0\end{cases} \\
& Q 36 d_{n 3, h 6}=\frac{2}{\tau_{y 3}} \cdot \lambda 3_{n 3} \cdot \begin{cases}{\left[y_{2} \cdot F_{s}\left(\lambda 3_{n 3}, y_{2}, y_{2}, \tau_{y 3}\right)-F_{l s}\left(\lambda 3_{n 3}, y_{2}, y_{2}, \tau_{y 3}\right)\right]} & \text { for } h 6=0 \\
\frac{-1}{\beta 6_{h 6}} \cdot \operatorname{csch}\left(\beta 6_{h 6} \cdot \tau_{y 6}\right) \cdot F_{s h s}\left(\beta 6_{h 6}, \lambda 3_{n 3}, y_{2}, y_{2}, y_{2}, \tau_{y 3}\right) & \text { for } h 6 \neq 0\end{cases} \\
& Q 36 e_{n 3, n 6}=\frac{2}{\tau_{y 3}} \cdot \frac{\lambda 3_{n 3}}{\lambda 6_{n 6}} \cdot \operatorname{csch}\left(\lambda 6_{n 6} \cdot \tau_{x 6}\right) \cdot F_{s s}\left(\lambda 6_{n 6}, \lambda 3_{n 3}, y_{2}, y_{2}, y_{2}, \tau_{y 3}\right) \text {, } \\
& Q 36 f_{n 3, n 6}=-\frac{2}{\tau_{y 3}} \cdot \frac{\lambda 3_{n 3}}{\lambda 6_{n 6}} \cdot \operatorname{coth}\left(\lambda 6_{n 6} \cdot \tau_{x 6}\right) \cdot F_{s S}\left(\lambda 6_{n 6}, \lambda 3_{n 3}, y_{2}, y_{2}, y_{2}, \tau_{y 3}\right) \text {, } \\
& E S 36_{n 3}=-\mu_{6} \cdot J_{z 6} \cdot \frac{\lambda 3_{n 3}}{\tau_{y 3}} \cdot F_{l 2 s}\left(\lambda 3_{n 3}, y_{2}, y_{2}, \tau_{y 3}\right) \text {. }
\end{aligned}
$$

Appendix C.5. Expression of $c 4_{h 4^{\prime}}^{x} d 4_{h 4}^{x}$ and $e 4_{n 4}^{y}$ for Region 4

By using (4), the development of (20a) gives

$$
\begin{gathered}
c 4_{h 4}^{x}=-\sum_{h 1=1}^{\infty} d 1_{h 1}^{x} \cdot Q 41 d_{h 4, h 1}, \\
Q 41 d_{h 4, h 1}=-\frac{2}{\tau_{x 4}} \cdot \frac{\beta 4_{h 4}}{\beta 1_{h 1}} \cdot \operatorname{th}\left(\beta 1_{h 1} \cdot \tau_{y 1}\right) \cdot F_{S S}\left(\beta 1_{h 1}, \beta 4_{h 4}, x_{1}, x_{5}, x_{5}, \tau_{x 4}\right) .
\end{gathered}
$$

By using (8), the development of (20b) gives

$$
\begin{gathered}
d 4_{h 4}^{x}=-\sum_{h 2=1}^{\infty} c 2_{h 2}^{x} \cdot Q 42 c_{h 4, h 2}, \\
Q 42 c_{h 4, h 2}=\frac{2}{\tau_{x 4}} \cdot \frac{\beta 4_{h 4}}{\beta 2_{h 2}} \cdot \operatorname{th}\left(\beta 2_{h 2} \cdot \tau_{y 2}\right) \cdot F_{s s}\left(\beta 2_{h 2}, \beta 4_{h 4}, x_{1}, x_{5}, x_{5}, \tau_{x 4}\right) .
\end{gathered}
$$

By using (32), the development of (21) gives

$$
\begin{gathered}
e 4_{n 4}^{y}=E S 47_{n 4}-\mid \begin{array}{ll}
\sum_{h 7=0}^{\infty}\left(c 7_{h 7}^{x} \cdot Q 47 c_{n 4, h 7}+d 7_{h 7}^{x} \cdot Q 47 d_{n 4, h 7}\right) \\
\cdots+\sum_{n 7=1}^{\infty}\left(e 7_{n 7}^{y} \cdot Q 47 e_{n 4, n 7}+f 7_{n 7}^{y} \cdot Q 47 f_{n 4, n 7}\right)
\end{array}, \\
Q 47 c_{n 4, h 7}=\frac{2}{\tau_{y 4}} \cdot \lambda 4_{n 4} \cdot \begin{cases}-\left[y_{3} \cdot F_{s}\left(\lambda 4_{n 4}, y_{2}, y_{2}, \tau_{y 4}\right)-F_{l s}\left(\lambda 4_{n 4}, y_{2}, y_{2}, \tau_{y 4}\right)\right] & \text { for } h 7=0 \\
\frac{1}{\beta_{h 7}} \cdot \frac{\cos \left(\beta 7_{h 7} \cdot \tau_{x 7}\right)}{\operatorname{sh}\left(\beta 7_{h 7} \cdot \tau_{y 7}\right)} \cdot F_{s h s}\left(\beta 7_{h 7}, \lambda 4_{n 4}, y_{3}, y_{2}, y_{2}, \tau_{y 4}\right) & \text { for } h 7 \neq 0\end{cases}
\end{gathered}
$$




$$
\begin{gathered}
Q 47 d_{n 4, h 7}=\frac{2}{\tau_{y 4}} \cdot \lambda 4_{n 4} \cdot \begin{cases}{\left[y_{2} \cdot F_{s}\left(\lambda 4_{n 4}, y_{2}, y_{2}, \tau_{y 4}\right)-F_{l s}\left(\lambda 4_{n 4}, y_{2}, y_{2}, \tau_{y 4}\right)\right]} & \text { for } h 7=0 \\
\frac{-1}{\beta_{h 7}} \cdot \frac{\cos \left(\beta 7_{h 7} \cdot \tau_{x 7}\right)}{\operatorname{sh}\left(\beta 7_{h 7} \cdot \tau_{y 7}\right)} \cdot F_{s h s}\left(\beta 7_{h 7}, \lambda 4_{n 4}, y_{2}, y_{2}, y_{2}, \tau_{y 4}\right) & \text { for } h 7 \neq 0\end{cases} \\
Q 47 e_{n 4, n 7}=\frac{2}{\tau_{y 4}} \cdot \frac{\lambda 4_{n 4}}{\lambda 7_{n 7}} \cdot \operatorname{coth}\left(\lambda 7_{n 7} \cdot \tau_{x 7}\right) \cdot F_{s s}\left(\lambda 7_{n 7}, \lambda 4_{n 4}, y_{2}, y_{2}, y_{2}, \tau_{y 4}\right), \\
Q 47 f_{n 4, n 7}=-\frac{2}{\tau_{y 4}} \cdot \frac{\lambda 4_{n 4}}{\lambda 7_{n 7}} \cdot \operatorname{csch}\left(\lambda 7_{n 7} \cdot \tau_{x 7}\right) \cdot F_{s s}\left(\lambda 7_{n 7}, \lambda 4_{n 4}, y_{2}, y_{2}, y_{2}, \tau_{y 4}\right), \\
E S 47_{n 4}=-\mu_{7} \cdot J_{z 7} \cdot \frac{\lambda 4_{n 4}}{\tau_{y 4}} \cdot F_{l 2 s}\left(\lambda 4_{n 4}, y_{2}, y_{2}, \tau_{y 4}\right) .
\end{gathered}
$$

Appendix C.6. Expression of $c 5_{h 5}^{x}, d 5_{h 5}^{x}, e 5_{n 5}^{y}$ and $f 5_{n 5}^{y}$ for Region 5

By using (4), the development of (25a) gives

$$
\begin{gathered}
c 5_{h 5}^{x}=-\sum_{h 1=1}^{\infty} d 1_{h 1}^{x} \cdot Q 51 d_{h 5, h 1}, \\
Q 51 d_{h 5, h 1}=-\frac{2}{\tau_{x 5}} \cdot \frac{\beta 5_{h 5}}{\beta 1_{h 1}} \cdot \operatorname{th}\left(\beta 1_{h 1} \cdot \tau_{y 1}\right) \cdot F_{S s}\left(\beta 1_{h 1}, \beta 5_{h 5}, x_{1}, x_{3}, x_{3}, \tau_{x 5}\right) .
\end{gathered}
$$

By using (8), the development of (25b) gives

$$
\begin{gathered}
d 5_{h 5}^{x}=-\sum_{h 2=1}^{\infty} c 2_{h 2}^{x} \cdot Q 52 c_{h 5, h 2}, \\
Q 52 c_{h 5, h 2}=\frac{2}{\tau_{x 5}} \cdot \frac{\beta 5_{h 5}}{\beta 2_{h 2}} \cdot \operatorname{th}\left(\beta 2_{h 2} \cdot \tau_{y 2}\right) \cdot F_{s s}\left(\beta 2_{h 2}, \beta 5_{h 5}, x_{1}, x_{3}, x_{3}, \tau_{x 5}\right) .
\end{gathered}
$$

By using (27), the development of (26a) gives

$$
\begin{gathered}
e 5_{n 5}^{y}=E S 56_{n 5}-\mid \begin{array}{ll}
\sum_{h 6=0}^{\infty}\left(c 6_{h 6}^{x} \cdot Q 56 c_{n 5, h 6}+d 6_{h 6}^{x} \cdot Q 56 d_{n 5, h 6}\right) \\
\cdots+\sum_{n 6=1}^{\infty}\left(e 6_{n 6}^{y} \cdot Q 56 e_{n 5, n 6}+f 6_{n 6}^{y} \cdot Q 56 f_{n 5, n 6}\right)
\end{array}, \\
Q 56 c_{n 5, h 6}=\frac{2}{\tau_{y 5}} \cdot \lambda 5_{n 5} \cdot \begin{cases}-\left[y_{3} \cdot F_{s}\left(\lambda 5_{n 5}, y_{2}, y_{2}, \tau_{y 5}\right)-F_{l s}\left(\lambda 5_{n 5}, y_{2}, y_{2}, \tau_{y 5}\right)\right] & \text { for } h 6=0 \\
\frac{1}{\beta 6_{h 6}} \cdot \frac{\cos \left(\beta 6_{h 6} \cdot \tau_{x 6}\right)}{\operatorname{sh}\left(\beta 6_{h 6} \cdot \tau_{y 6}\right)} \cdot F_{s h s}\left(\beta 6_{h 6}, \lambda 5_{n 5}, y_{3}, y_{2}, y_{2}, \tau_{y 5}\right) & \text { for } h 6 \neq 0\end{cases} \\
Q 56 d_{n 5, h 6}=\frac{2}{\tau_{y 5}} \cdot \lambda 5_{n 5} \cdot \begin{cases}{\left[y_{2} \cdot F_{s}\left(\lambda 5_{n 5}, y_{2}, y_{2}, \tau_{y 5}\right)-F_{l s}\left(\lambda 5_{n 5}, y_{2}, y_{2}, \tau_{y 5}\right)\right]} & \text { for } h 6=0 \\
-\frac{1}{\beta 6_{h 6}} \frac{\cos \left(\beta 6_{h 6} \cdot \tau_{x 6}\right)}{\operatorname{sh}\left(\beta 6_{h 6} \cdot \tau_{y 6}\right)} \cdot F_{s h s}\left(\beta 6_{h 6}, \lambda 5_{n 5}, y_{2}, y_{2}, y_{2}, \tau_{y 5}\right) & \text { for } h 6 \neq 0\end{cases} \\
Q 56 e_{n 5, n 6}=\frac{2}{\tau_{y 5}} \cdot \frac{\lambda 5_{n 5}}{\lambda 6_{n 6}} \cdot \operatorname{coth}\left(\lambda 6_{n 6} \cdot \tau_{x 6}\right) \cdot F_{s s}\left(\lambda 6_{n 6}, \lambda 5_{n 5}, y_{2}, y_{2}, y_{2}, \tau_{y 5}\right), \\
Q 56 f_{n 5, n 6}=-\frac{2}{\tau_{y 5}} \cdot \frac{\lambda 5_{n 5}}{\lambda 6_{n 6}} \cdot \operatorname{csch}\left(\lambda 6_{n 6} \cdot \tau_{x 6}\right) \cdot F_{s s}\left(\lambda 6_{n 6}, \lambda 5_{n 5}, y_{2}, y_{2}, y_{2}, \tau_{y 5}\right), \\
E S 56_{n 5}=-\mu_{6} \cdot J_{z 6} \cdot \frac{\lambda 5_{n 5}}{\tau_{y 5}} \cdot F_{l 2 s}\left(\lambda 5_{n 5}, y_{2}, y_{2}, \tau_{y 5}\right) .
\end{gathered}
$$

By using (32), the development of (26b) gives

$$
f 5_{n 5}^{y}=E S 57_{n 5}-\mid \begin{aligned}
& \sum_{h 7=0}^{\infty}\left(c 7_{h 7}^{x} \cdot Q 57 c_{n 5, h 7}+d 7_{h 7}^{x} \cdot Q 57 d_{n 5, h 7}\right) \\
& \cdots+\sum_{n 7=1}^{\infty}\left(e 7_{n 7}^{y} \cdot Q 57 e_{n 5, n 7}+f 7_{n 7}^{y} \cdot Q 57 f_{n 5, n 7}\right)
\end{aligned},
$$




$$
\begin{gathered}
Q 57 c_{n 5, h 7}=\frac{2}{\tau_{y 5}} \cdot \lambda 5_{n 5} \cdot \begin{cases}-\left[y_{3} \cdot F_{s}\left(\lambda 5_{n 5}, y_{2}, y_{2}, \tau_{y 5}\right)-F_{l s}\left(\lambda 5_{n 5}, y_{2}, y_{2}, \tau_{y 5}\right)\right] & \text { for } h 7=0 \\
\frac{1}{\beta 7_{h 7}} \cdot \operatorname{csch}\left(\beta 7_{h 7} \cdot \tau_{y 7}\right) \cdot F_{s h s}\left(\beta 7_{h 7}, \lambda 5_{n 5}, y_{3}, y_{2}, y_{2}, \tau_{y 5}\right) & \text { for } h 7 \neq 0\end{cases} \\
Q 57 d_{n 5, h 7}=\frac{2}{\tau_{y 5}} \cdot \lambda 5_{n 5} \cdot \begin{cases}{\left[y_{2} \cdot F_{s}\left(\lambda 5_{n 5}, y_{2}, y_{2}, \tau_{y 5}\right)-F_{l s}\left(\lambda 5_{n 5}, y_{2}, y_{2}, \tau_{y 5}\right)\right]} & \text { for } h 7=0 \\
-\frac{1}{\beta 7_{h 7}} \cdot \operatorname{csch}\left(\beta 7_{h 7} \cdot \tau_{y 7}\right) \cdot F_{s h s}\left(\beta 7_{h 7}, \lambda 5_{n 5}, y_{2}, y_{2}, y_{2}, \tau_{y 5}\right) & \text { for } h 7 \neq 0\end{cases} \\
Q 57 e_{n 5, n 7}=\frac{2}{\tau_{y 5}} \cdot \frac{\lambda 5_{n 5}}{\lambda 7_{n 7}} \cdot \operatorname{csch}\left(\lambda 7_{n 7} \cdot \tau_{x 7}\right) \cdot F_{s s}\left(\lambda 7_{n 7}, \lambda 5_{n 5}, y_{2}, y_{2}, y_{2}, \tau_{y 5}\right), \\
Q 57 f_{n 5, n 7}=-\frac{2}{\tau_{y 5}} \cdot \frac{\lambda 5_{n 5}}{\lambda 7_{n 7}} \cdot \operatorname{coth}\left(\lambda 7_{n 7} \cdot \tau_{x 7}\right) \cdot F_{s s}\left(\lambda 7_{n 7}, \lambda 5_{n 5}, y_{2}, y_{2}, y_{2}, \tau_{y 5}\right), \\
E S 57_{n 5}=-\mu_{7} \cdot J_{z 7} \cdot \frac{\lambda 5_{n 5}}{\tau_{y 5}} \cdot F_{l 2 s}\left(\lambda 5_{n 5}, y_{2}, y_{2}, \tau_{y 5}\right) .
\end{gathered}
$$

Appendix C.7. Expression of $c 6_{0}^{x}, d 6_{0}^{x}, c 6_{h 6^{\prime}}^{x}, d 6_{h 6^{\prime}}^{x}, e 6_{n 6}^{y}$ and $f 6_{n 6}^{y}$ for Region 6

By using (4) and (27d), the development of (30a) and (30b) gives

$$
\begin{gathered}
c 6_{h 6}^{x}=E S 61_{h 6}-\sum_{h 1=1}^{\infty} d 1_{h 1}^{x} \cdot Q 61 d_{h 6, h 1}, \\
Q 61 d_{h 6, h 1}=-\frac{1}{\tau_{x 6}} \cdot \frac{1}{\beta 1_{h 1}} \cdot t h\left(\beta 1_{h 1} \cdot \tau_{y 1}\right) \cdot \begin{cases}\frac{1}{\tau_{y 6}} \cdot F_{S}\left(\beta 1_{h 1}, x_{1}, x_{2}, \tau_{x 6}\right) & \text { for } h 6=0 \\
2 \cdot \beta 6_{h 6} \cdot F_{c S}\left(\beta 6_{h 6}, \beta 1_{h 1}, x_{2}, x_{1}, x_{2}, \tau_{x 6}\right) & \text { for } h 6 \neq 0\end{cases} \\
E S 61_{h 6}= \begin{cases}-\left.\frac{1}{\tau_{y 6}} \cdot A_{z P 6}\right|_{y=y_{2}} & \text { for } h 6=0 \\
0 & \text { for } h 6 \neq 0\end{cases}
\end{gathered}
$$

By using (8) and (27d), the development of (30c) and (30d) gives

$$
\begin{gathered}
d 6_{h 6}^{x}=E S 62_{h 6}-\sum_{h 2=1}^{\infty} c 2_{h 2}^{x} \cdot Q 62 c_{h 6, h 2}, \\
Q 62 c_{h 6, h 2}=\frac{1}{\tau_{x 6}} \cdot \frac{1}{\beta 22_{h 2}} \cdot \operatorname{th}\left(\beta 2_{h 2} \cdot \tau_{y 2}\right) \cdot \begin{cases}\frac{1}{\tau_{y 6}} \cdot F_{S}\left(\beta 2_{h 2}, x_{1}, x_{2}, \tau_{x 6}\right) & \text { for } h 6=0 \\
2 \cdot \beta 6_{h 6} \cdot F_{c s}\left(\beta 6_{h 6}, \beta 2_{h 2}, x_{2}, x_{1}, x_{2}, \tau_{x 6}\right) & \text { for } h 6 \neq 0\end{cases} \\
E S 62_{h 6}= \begin{cases}-\left.\frac{1}{\tau_{y 6}} \cdot A_{z P 6}\right|_{y=y_{3}} & \text { for } h 6=0 \\
0 & \text { for } h 6 \neq 0\end{cases}
\end{gathered}
$$

By using (24), the development of (31a) gives

$$
\begin{gathered}
e 6_{n 6}^{y}=-\mid \begin{array}{l}
\sum_{h 5=1}^{\infty}\left(c 5_{h 5}^{x} \cdot Q 65 c_{n 6, h 5}+d 5_{h 5}^{x} \cdot Q 65 d_{n 6, h 5}\right) \\
\cdots+\sum_{n 5=1}^{\infty}\left(e 5_{n 5}^{y} \cdot Q 65 e_{n 6, n 5}+f 5_{n 5}^{y} \cdot Q 65 f_{n 6, n 5}\right)
\end{array}, \\
Q 65 c_{n 6, h 5}=-\frac{2}{\tau_{y 6}} \cdot \frac{\mu_{6}}{\mu_{5}} \cdot \operatorname{csch}\left(\beta 5_{h 5} \cdot \tau_{y 5}\right) \cdot F_{s h s}\left(\beta 5_{h 5}, \lambda 6_{n 6}, y_{3}, y_{2}, y_{2}, \tau_{y 6}\right), \\
Q 65 d_{n 6, h 5}=\frac{2}{\tau_{y 6}} \cdot \frac{\mu_{6}}{\mu_{5}} \cdot \operatorname{csch}\left(\beta 5_{h 5} \cdot \tau_{y 5}\right) \cdot F_{s h s}\left(\beta 5_{h 5}, \lambda 6_{n 6}, y_{2}, y_{2}, y_{2}, \tau_{y 6}\right), \\
Q 65 e_{n 6, n 5}=-\frac{2}{\tau_{y 6}} \cdot \frac{\mu_{6}}{\mu_{5}} \cdot \operatorname{coth}\left(\lambda 5_{n 5} \cdot \tau_{x 5}\right) \cdot F_{s s}\left(\lambda 5_{n 5}, \lambda 6_{n 6}, y_{2}, y_{2}, y_{2}, \tau_{y 6}\right), \\
Q 65 f_{n 6, n 5}=\frac{2}{\tau_{y 6}} \cdot \frac{\mu_{6}}{\mu_{5}} \cdot \operatorname{csch}\left(\lambda 5_{n 5} \cdot \tau_{x 5}\right) \cdot F_{s s}\left(\lambda 5_{n 5}, \lambda 6_{n 6}, y_{2}, y_{2}, y_{2}, \tau_{y 6}\right),
\end{gathered}
$$


By using (14), the development of (31b) gives

$$
\begin{gathered}
f 6_{n 6}^{y}=-\mid \begin{array}{l}
\sum_{h 3=1}^{\infty}\left(c 3_{h 3}^{x} \cdot Q 63 c_{n 6, h 3}+d 3_{h 3}^{x} \cdot Q 63 d_{n 6, h 3}\right) \\
\cdots+\sum_{n 3=1}^{\infty} f 3_{n 3}^{y} \cdot Q 63 f_{n 6, n 3}
\end{array} \\
Q 63 c_{n 6, h 3}=-\frac{2}{\tau_{y 6}} \cdot \frac{\mu_{6}}{\mu_{3}} \cdot \frac{\cos \left(\beta 3_{h 3} \cdot \tau_{x 3}\right)}{\operatorname{sh}\left(\beta 3_{h 3} \cdot \tau_{y 3}\right)} \cdot F_{s h s}\left(\beta 3_{h 3}, \lambda 6_{n 6}, y_{3}, y_{2}, y_{2}, \tau_{y 6}\right), \\
Q 63 d_{n 6, h 3}=\frac{2}{\tau_{y 6}} \cdot \frac{\mu_{6}}{\mu_{3}} \cdot \frac{\cos \left(\beta 3_{h 3} \cdot \tau_{x 3}\right)}{\operatorname{sh}\left(\beta 3_{h 3} \cdot \tau_{y 3}\right)} \cdot F_{s h s}\left(\beta 3_{h 3}, \lambda 6_{n 6}, y_{2}, y_{2}, y_{2}, \tau_{y 6}\right), \\
Q 63 f_{n 6, n 3}=\frac{2}{\tau_{y 6}} \cdot \frac{\mu_{6}}{\mu_{3}} \cdot \operatorname{coth}\left(\lambda 3_{n 3} \cdot \tau_{x 3}\right) \cdot F_{s s}\left(\lambda 3_{n 3}, \lambda 6_{n 6}, y_{2}, y_{2}, y_{2}, \tau_{y 6}\right) .
\end{gathered}
$$

Appendix C.8. Expression of $c 7_{0}^{x}, d 7_{0}^{x}, c 7_{h 7}^{x}, d 7_{h 7}^{x}, e 7_{n 7}^{y}$ and $f 7_{n 7}^{y}$ for Region 7

By using (4) and (32d), the development of (35a) and (35b) gives

$$
\begin{gathered}
c 7_{h 7}^{x}=E S 71_{h 7}-\sum_{h 1=1}^{\infty} d 1_{h 1}^{x} \cdot Q 71 d_{h 7, h 1} \\
Q 71 d_{h 7, h 1}=-\frac{1}{\tau_{x 7}} \cdot \frac{1}{\beta 1_{h 1}} \cdot t h\left(\beta 1_{h 1} \cdot \tau_{y 1}\right) \cdot \begin{cases}\frac{1}{\tau_{y 7}} \cdot F_{S}\left(\beta 1_{h 1}, x_{1}, x_{4}, \tau_{x 7}\right) & \text { for } h 7=0 \\
2 \cdot \beta 7_{h 7} \cdot F_{c S}\left(\beta 7_{h 7}, \beta 1_{h 1}, x_{4}, x_{1}, x_{4}, \tau_{x 7}\right) & \text { for } h 7 \neq 0\end{cases} \\
E S 71_{h 7}= \begin{cases}-\left.\frac{1}{\tau_{y 7}} \cdot A_{z P 7}\right|_{y=y_{2}} & \text { for } h 7=0 \\
0 & \text { for } h 7 \neq 0\end{cases}
\end{gathered}
$$

By using (8) and (32d), the development of (35c) and (35d) gives

$$
\begin{aligned}
& d 7_{h 7}^{x}=E S 72_{h 7}-\sum_{h 2=1}^{\infty} c 2_{h 2}^{x} \cdot Q 72 c_{h 7, h 2} \\
& Q 72 c_{h 7, h 2}=\frac{1}{\tau_{x 7}} \cdot \frac{1}{\beta 2_{h 2}} \cdot \operatorname{th}\left(\beta 2_{h 2} \cdot \tau_{y 2}\right) \cdot \begin{cases}\frac{1}{\tau_{y 7}} \cdot F_{S}\left(\beta 2_{h 2}, x_{1}, x_{4}, \tau_{x 7}\right) & \text { for } h 7=0 \\
2 \cdot \beta 7_{h 7} \cdot F_{c S}\left(\beta 7_{h 7}, \beta 2_{h 2}, x_{4}, x_{1}, x_{4}, \tau_{x 7}\right) & \text { for } h 7 \neq 0\end{cases} \\
& E S 72_{h 7}= \begin{cases}-\left.\frac{1}{\tau_{y 7}} \cdot A_{z P 7}\right|_{y=y_{3}} & \text { for } h 7=0 \\
0 & \text { for } h 7 \neq 0\end{cases}
\end{aligned}
$$

By using (24), the development of (36a) gives

$$
\begin{gathered}
f 7_{n 7}^{y}=-\mid \begin{array}{l}
\sum_{h 5=1}^{\infty}\left(c 5_{h 5}^{x} \cdot Q 75 c_{n 7, h 5}+d 5_{h 5}^{x} \cdot Q 75 d_{n 7, h 5}\right) \\
\cdots+\sum_{n 5=1}^{\infty}\left(e 5_{n 5}^{y} \cdot Q 75 e_{n 7, n 5}+f 5_{n 5}^{y} \cdot Q 75 f_{n 7, n 5}\right)
\end{array}, \\
Q 75 c_{n 7, h 5}=-\frac{2}{\tau_{y 7}} \cdot \frac{\mu_{7}}{\mu_{5}} \cdot \frac{\cos \left(\beta 5_{h 5} \cdot \tau_{x 5}\right)}{\operatorname{sh}\left(\beta 5_{h 5} \cdot \tau_{y 5}\right)} \cdot F_{s h s}\left(\beta 5_{h 5}, \lambda 7_{n 7}, y_{3}, y_{2}, y_{2}, \tau_{y 7}\right), \\
Q 75 d_{n 7, h 5}=\frac{2}{\tau_{y 7}} \cdot \frac{\mu_{7}}{\mu_{5}} \cdot \frac{\cos \left(\beta 5_{h 5} \cdot \tau_{x 5}\right)}{\operatorname{sh}\left(\beta 5_{h 5} \cdot \tau_{y 5}\right)} \cdot F_{s h s}\left(\beta 5_{h 5}, \lambda 7_{n 7}, y_{2}, y_{2}, y_{2}, \tau_{y 7}\right), \\
Q 75 e_{n 7, n 5}=-\frac{2}{\tau_{y 7}} \cdot \frac{\mu_{7}}{\mu_{5}} \cdot \operatorname{csch}\left(\lambda 5_{n 5} \cdot \tau_{x 5}\right) \cdot F_{s s}\left(\lambda 5_{n 5}, \lambda 7_{n 7}, y_{2}, y_{2}, y_{2}, \tau_{y 7}\right),
\end{gathered}
$$




$$
Q 75 f_{n 7, n 5}=\frac{2}{\tau_{y 7}} \cdot \frac{\mu_{7}}{\mu_{5}} \cdot \operatorname{coth}\left(\lambda 5_{n 5} \cdot \tau_{x 5}\right) \cdot F_{s s}\left(\lambda 5_{n 5}, \lambda 7_{n 7}, y_{2}, y_{2}, y_{2}, \tau_{y 7}\right) .
$$

By using (19), the development of (36b) gives

$$
\begin{gathered}
e 7_{n 7}^{y}=-\mid \begin{array}{l}
\sum_{h 4=1}^{\infty}\left(c 4_{h 4}^{x} \cdot Q 74 c_{n 7, h 4}+d 4_{h 4}^{x} \cdot Q 74 d_{n 7, h 4}\right) \\
\cdots+\sum_{n 4=1}^{\infty} e 4_{n 4}^{y} \cdot Q 74 e_{n 7, n 4}
\end{array} \\
Q 74 c_{n 7, h 4}=-\frac{2}{\tau_{y 7}} \cdot \frac{\mu_{7}}{\mu_{4}} \cdot \operatorname{csch}\left(\beta 4_{h 4} \cdot \tau_{y 4}\right) \cdot F_{s h s}\left(\beta 4_{h 4}, \lambda 7_{n 7}, y_{3}, y_{2}, y_{2}, \tau_{y 7}\right), \\
Q 74 d_{n 7, h 4}=\frac{2}{\tau_{y 7}} \cdot \frac{\mu_{7}}{\mu_{4}} \cdot \operatorname{csch}\left(\beta 4_{h 4} \cdot \tau_{y 4}\right) \cdot F_{s h s}\left(\beta 4_{h 4}, \lambda 7_{n 7}, y_{2}, y_{2}, y_{2}, \tau_{y 7}\right), \\
Q 74 e_{n 7, n 4}=-\frac{2}{\tau_{y 7}} \cdot \frac{\mu_{7}}{\mu_{4}} \cdot \operatorname{coth}\left(\lambda 4_{n 4} \cdot \tau_{x 4}\right) \cdot F_{s s}\left(\lambda 4_{n 4}, \lambda 7_{n 7}, y_{2}, y_{2}, y_{2}, \tau_{y 7}\right) .
\end{gathered}
$$

\section{References}

1. Yilmaz, M.; Krein, P.T. Capabilities of finite element analysis and magnetic equivalent circuits for electrical machine analysis and design. In Proceedings of the 2008 Power Electronics Specialists Conference (PESC), Rhodes, Greece, 15-19 June 2008.

2. Dubas, F.; Espanet, C. Analytical solution of the magnetic field in permanent-magnet motors taking into account slotting effect: No-load vector potential and flux density calculation. IEEE Trans. Magn. 2009, 45, 2097-2109.

3. Zhu, Z.Q.; Wu, L.J.; Xia, Z.P. An accurate subdomain model for magnetic field computation in slotted surface-mounted permanent-magnet machines. IEEE Trans. Magn. 2010, 46, 1100-1115.

4. Rahideh, A.; Korakianitis, T. Analytical calculation of open-circuit magnetic field distribution of slotless brushless PM machines. Electr. Power Energy Syst. 2012, 44, 99-114.

5. Tiegna, H.; Amara, Y.; Barakat, G. Overview of analytical models of permanent magnet electrical machines for analysis and design purposes. Math. Comput. Simul. 2013, 90, 162-177.

6. Curti, M.; Paulides, J.J.H.; Lomonova, E.A. An overview of analytical methods for magnetic field computation. In Proceedings of the Tenth International Conference on Ecological Vehicles and Renewable Energies (EVER), Monte Carlo, Monaco, 31 March-2 April 2015.

7. Lehmann, T. Méthode graphique pour déterminer le trajet des lignes de force dans l'air. Revue d'Électricité: La Lumière Électrique 1909, 43-45, 103-110; 137-142; 163-168.

8. Flux2D. General Operating Instructions; Version 10.2.1; Cedrat S.A. Electrical Engineering: Grenoble, France, 2008.

9. Jin, J. The Finite Element Method in Electromagnetic, 2nd ed.; John Wiley \& Sons, Inc.: New York, NY, USA, 2002.

10. Stoll, R.L. The Analysis of Eddy Currents; Clarendon Press: Oxford, UK, 1974.

11. Smith, G.D. Numerical Solution of Partial Differential Equations: Finite Difference Methods, 3rd ed.; Clarendon Press: Oxford, UK, 1985.

12. Wrobel, L.C.; Aliabadi, M.H. The Boundary Element Method; John Wiley \& Sons, Inc.: New York, NY, USA, 2002.

13. Alger, P.L. Induction Machines: Their Behavior and Uses; Gordon and Breach Publisher: London, UK, 1970.

14. Holman, J.P. Heat Transfer, 6th ed.; McGraw-Hill Book Compagny: New York, NY, USA, 1986.

15. Roters, H.C. Electromagnetic Devices; John Wiley \& Sons, Inc.: New York, NY, USA, 1941.

16. Ostovic, V. Dynamics of Saturated Electric Machines; Springer-Verlag: New York, NY, USA, 1989.

17. Driscoll, T.A.; Trefethen, L.N. Schwarz-Christoffel Mapping; Cambridge University Press: Cambridge, UK, 2002.

18. Sylvester, P. Modern Electromagnetic Fields; Prentice-Hall: London, UK, 1968.

19. Binns, K.J.; Lawrenson, P.J.; Trowbridge, C.W. The Analytical and Numerical Solution of Electric and Magnetic Fields; John Wiley \& Sons, Inc.: New York, NY, USA, 1992.

20. Hague, B. Electromagnetic Problems in Electrical Engineering; Oxford University Press: London, UK, 1929.

21. Melcher, J.R. Continuum Electromechanics; MIT Press: Cambridge, MA, USA, 1981. 
22. Farlow, S.J. Partial Differential Equations for Scientists and Engineers; Dover, Inc.: New York, NY, USA, 1993.

23. Schutte, J.; Strauss, J.M. Optimisation of a transverse flux linear PM generator using 3D Finite Element Analysis. In Proceedings of the 2010 XIX International Conference on Electrical Machines (ICEM), Rome, Italy, 6-8 September 2010.

24. Espanet, C.; Kieffer, C.; Mira, A.; Giurgea, S.; Gustin, F. Optimal design of a special permanent magnet synchronous machine for magnetocaloric refrigeration. In Proceedings of the 2013 IEEE Energy Conversion Congress and Exposition (ECCE), Denver, CO, USA, 15-19 September 2013.

25. Ede, J.D.; Atallah, K.; Jewel, G.W.; Wang, J.B.; Howe, D. Effect of axial segmentation of permanent magnets on rotor loss in modular permanent-magnet brushless machines. IEEE Trans. Ind. Appl. 2007, 43, 1207-1213.

26. Vansompel, H.; Sergeant, P.; Dupré, L. A multilayer 2-D-2-D coupled model for eddy current calculation in the rotor of an axial-flux PM machine. IEEE Trans. Energy Conv. 2012, 27, 784-791.

27. Benlamine, R.; Dubas, F.; Randi, S-A.; Lhotellier, D.; Espanet, C. 3-D numerical hybrid method for PM eddy-current losses calculation: Application to axial-flux PMSMs. IEEE Trans. Magn. 2015, 51, doi:10.1109/TMAG.2015.2405053.

28. Carter, F.W. Air-gap induction. Electr. World Eng. 1901, XXXVIII, 884-888.

29. Kumar, P.; Bauer, P. Improved analytical model of a permanent-magnet brushless DC motor. IEEE Trans. Magn. 2008, 44, 2299-2309.

30. Dalal, A.; Kumar, P. Analytical model of a permanent magnet Brushless DC Motor with non-linear ferromagnetic material. In Proceedings of the 2014 International Conference on Electrical Machines (ICEM), Berlin, Germany, 2-5 September 2014.

31. Dalal, A.; Kumar, P. Analytical model for permanent magnet motor with slotting effect, armature reaction, and ferromagnetic material property. IEEE Trans. Magn. 2015, 51, doi:10.1109/TMAG.2015.2459036.

32. Boules, N. Two-dimensional field analysis of cylindrical machines with permanent magnet excitation. IEEE Trans. Ind. Appl. 1984, IA-20, 1267-1277.

33. Moallem, M.; Madhkhan, M. Predicting the parameters and performance of brushless DC motor including saturation and slotting effects and magnetic circuit variations. In Proceedings of the 1995 International Conference on Power Electronics and Drive Systems (PEDS), Singapore, 21-24 February 1995.

34. Abbaszadeh, K.; Alam, F.R. On-load field component separation in surface-mounted permanent magnet motors using an improved conformal mapping method. IEEE Trans. Magn. 2016, 52, doi:10.1109/TMAG.2015.2493150.

35. Berkani, M.S.; Sough, M.L.; Giurgea, S.; Dubas, F.; Boualem, B.; Espanet, C. A simple analytical approach to model saturation in surface mounted permanent magnet synchronous motors. In Proceedings of the 2015 IEEE Energy Conversion Congress and Exposition (ECCE), Montreal, QC, Canada, 20-24 September 2015.

36. Mishkin, E. Theory of the squirrel-cage induction machine derived directly from Maxwell's field equations. Q. J. Mech. Appl. Math. 1954, 7, 472-487.

37. Cullen, A.L.; Barton, T.H. A simplified electromagnetic theory of the induction motor, using the concept of wave impedance. Proc. IEE 1958, 105C, 331-336.

38. Greig, J.; Freeman, E.M. Travelling-wave problem in electrical machines. Proc. IEE 1967, 114, 1681-1683.

39. Freeman, E.M. Travelling waves in induction machines: Input impedance and equivalent circuits. Proc. IEE 1968, 115, 1772-1776.

40. Jones, C.V.; Gibson, R.C. Correlation of the air-gap vector potential of an induction motor with the magnetising current. Proc. IEE 1969, 116, 385-390.

41. Williamson, $\mathrm{S}$. The anisotropic layer theory of induction machines and induction devices. IMA J. Appl. Math. $1967,17,69-84$.

42. Gieras, J. Analysis of multilayer rotor induction motor with higher space harmonics taken into account. Proc. IEE 1991, 138, 59-67.

43. Panaitescu, A.; Panaitescu, I. A field model for induction machines. In Proceedings of the International Conference on Electrical Machines (ICEM), Vigo, Spain, 10-12 September 1996.

44. Madescu, G.; Boldea, I.; Miller, T.J.E. An analytical iterative model (AIM) for induction motor design. In Proceedings of the Conference Record of the 1996 IEEE Industry Applications Conference Thirty-First IAS Annual Meeting, San Diego, CA, USA, 6-10 October 1996.

45. Sprangers, R.L.J.; Motoasca, T.E.; Lomonova, E.A. Extended anisotropic layer theory for electrical machines. IEEE Trans. Magn. 2013, 49, 2217-2220.

46. Sprangers, R.L.J.; Paulides, J.J.H.; Boynov, K.O.; Lomonova, E.A.; Waarma, J. Comparison of two anisotropic layer models applied to induction motors. IEEE Trans. Ind. Appl. 2014, 50, 2533-2543. 
47. Sprangers, R.L.J.; Paulides, J.J.H.; Gysen, B.L.J.; Lomonova, E.A. Magnetic saturation in semi-analytical harmonic modeling for electric machine analysis. IEEE Trans. Magn. 2016, 52, doi:10.1109/TMAG.2015.2480708.

48. Djelloul, K.Z.; Boughrara, K.; Ibtiouen, R.; Dubas, F. Nonlinear analytical calculation of magnetic field and torque of switched reluctance machines. In Proceedings of the CISTEM, Marrakech-Benguérir, Maroc, 26-28 October 2016.

49. Sprangers, R.L.J.; Paulides, J.J.H.; Gysen, B.L.J.; Waarma, J.; Lomonova, E.A. Semi-analytical framework for synchronous reluctance motor analysis including finite soft-magnetic material permeability. IEEE Trans. Magn. 2015, 51, doi:10.1109/TMAG.2015.2442419.

50. Theodoulidis, T.P. Model of ferrite-cored probes for eddy current nondestructive evaluation. J. Appl. Phys. 2003, 93, 3071-3078.

51. Theodoulidis, T.P.; Bowler, J. Eddy-current interaction of a long coil with a slot in a conductive plate. IEEE Trans. Magn. 2005, 41, 1238-1247.

52. Sakkaki, F.; Bayani, H. Solution to the problem of E-cored coil above a layered half-space using the method of truncated region eigenfunction expansion. J. Appl. Phys. 2012, 111, doi:10.1063/1.3675183.

53. Tytko, G.; Dziczkowski, L. E-cored coil with a circular air gap inside the core column used in eddy current testing. IEEE Trans. Magn. 2015, 51, doi:10.1109/TMAG.2015.2440420.

54. Abdel-Razek, A.A.; Coulomb, J.L.; Feliachi, M.; Sabonnadière, J.C. The calculation of electromagnetic torque in saturated electric machines within combined numerical and analytical solutions of the field equations. IEEE Trans. Magn. 1981, 17, 3250-3252.

55. Féliachi, M.; Coulomb, J.L.; Mansir, H. Second order air-gap element for the dynamic finite-element analysis of the electromagnetic field in electric machines. IEEE Trans. Magn. 1983, 19, 2300-2303.

56. Goby, F.; Razek, A. Numerical calculation of electromagnetic forces. Math. Comput. Simul. 1987, $29,343-350$.

57. Liu, Z.J.; Bi, C.; Tan, H.C.; Low, T.S. A combined numerical and analytical approach for magnetic field analysis of permanent magnet machines. IEEE Trans. Magn. 1995, 31, 1372-1375.

58. Mirzayee, M.; Mehrjerdi, H.; Tsurkerman, I. Analysis of a high-speed solid rotor induction motor using coupled analytical method and reluctance networks. In Proceedings of the 2005 IEEE/ACES International Conference on Wireless Communications and Applied Computational Electromagnetics (ACES), Honolulu, HI, USA, 3-7 April 2005.

59. Gholizad, H.; Mirsalim, M.; Mirzayee, M. Dynamic Analysis of Highly Saturated Switched Reluctance Motors Using Coupled Magnetic Equivalent Circuit and the Analytical Solution. In Proceedings of the 6th International Conference on Computational Electromagnetics (CEM), Aachen, Germany, 4-7 April 2006.

60. Mirzayee, M.; Mirsalim, M.; Gholizad, H.; Arani, S.J. Combined 3D Numerical and Analytical Computation Approach for Analysis and Design of High Speed Solid Iron rotor Induction Machines. In Proceedings of the 6th International Conference on Computational Electromagnetics (CEM), Aachen, Germany, 4-6 April 2006.

61. Ghoizad, H.; Mirsalim, M.; Mirzayee, M.; Cheng, W. Coupled magnetic equivalent circuits and the analytical solution in the air-gap of squirrel cage induction machines. Int. J. Appl. Electromagn. Mech. 2007, 25, 749-754.

62. Hemeida, A.; Sergeant, P. Analytical modeling of surface PMSM using a combined solution of Maxwell's equations and magnetic equivalent circuit. IEEE Trans. Magn. 2014, 50, doi:10.1109/TMAG.2014.2330801.

63. Laoubi, Y.; Dhifli, M.; Verez, G.; Amara, Y.; Barakat, G. Open circuit performance analysis of a permanent magnet linear machine using a new hybrid analytical model. IEEE Trans. Magn. 2015, 51, doi:10.1109/TMAG.2014.2361017.

64. Ouagued, S.; Diriyé, A.A.; Amara, Y.; Barakat, G. A General Framework Based on a Hybrid Analytical Model for the Analysis and Design of Permanent Magnet Machines. IEEE Trans. Magn. 2015, 51, doi:10.1109/TMAG.2015.2442214.

65. Pluk, K.J.W.; Jansen, J.W.; Lomonova, E.A. Hybrid analytical modeling: Fourier modeling combined with mesh-based magnetic equivalent circuits. IEEE Trans. Magn. 2015, 51, doi:10.1109/TMAG.2015.2419197.

66. Pluk, K.J.W.; Jansen, J.W.; Lomonova, E.A. 3-D hybrid analytical modeling: 3-D Fourier modeling combined with mesh-based 3-D magnetic equivalent circuits. IEEE Trans. Magn. 2015, 51, doi:10.1109/TMAG.2015.2455951.

67. Zhang, Z.; Xia, C.L.; Yan, Y.; Wang, H.M. Analytical field calculation of doubly fed induction generator with core saturation considered. In Proceedings of the 8th IET International Conference on Power Electronics, Machines and Drives (PEMD 2016), Glasgow, UK, 19-21 April 2016.

68. Dubas, F.; Rahideh, A. 2-D analytical PM eddy-current loss calculations in slotless PMSM equipped with surface-inset magnets. IEEE Trans. Magn. 2014, 50, doi:10.1109/TMAG.2013.2285525. 
69. Gysen, B.L.J.; Meessen, K.J.; Paulides, J.J.H.; Lomonova, E.A. General formulation of the electromagnetic field distribution in machines and devices using Fourier analysis. IEEE Trans. Magn. 2010, 46, 39-52.

70. Meessen, K.J.; Gysen, B.L.J.; Paulides, J.J.H.; Lomonova, E.A. General formulation of fringing in 3-D cylindrical structures using Fourier analysis. IEEE Trans. Magn. 2012, 48, 2307-2323.

71. Lee, S.W.; Jones, W.; Campbell, J. Convergence of numerical solution of iris-type discontinuity problems. IEEE Trans. Microw. Theory Tech. 1971, 19, 528-536.

72. Mittra, R.; Itoh, T.; Li, T.-S. Analytical and numerical studies of the relative convergence phenomenon arising in the solution of an integral equation by the moment method. IEEE Trans. Microw. Theory Tech. 1972, 20, 96-104.

73. Hewitt, E.; Hewitt, R. The Gibbs-Wibraham phenomenon: An episode in Fourier analysis. Arch. Hist. Exact Sci. 1979, 21, 129-160.

74. Dubas, F. Conception D'un Moteur Rapide à Aimants Permanents Pour L'entraînement de Compresseurs de Piles à Combustible. Ph.D. Thesis, University of Franche-Comté (UFC), Belfort, France, 2006.

(C) 2017 by the authors; licensee MDPI, Basel, Switzerland. This article is an open access article distributed under the terms and conditions of the Creative Commons Attribution (CC BY) license (http:/ / creativecommons.org/licenses/by/4.0/). 